\author{
Universidade de São Paulo \\ Instituto de Física
}

\title{
Estudo de cadeias quânticas de Heisenberg desordenadas com acoplamentos aperiódicos.
}

\author{
Helder Luciani Casa Grande
}

Orientador: Prof. Dr. André de Pinho Vieira

Tese de doutorado apresentada ao Instituto de Física para a obtenção do título de Doutor em Ciências.

Banca examinadora:

Prof. Dr. André de Pinho Vieira (orientador - IFUSP)

Prof. Dr. Silvio Roberto de Azevedo Salinas (IFUSP)

Prof. Dr. Mario Jose de Oliveira (IFUSP)

Prof ${ }^{a}$. Dra . Angsula Gosh (UFAM)

Prof. Dr. José Abel Hoyos Neto (IFSC/USP)

São Paulo - 2014 
FICHA CATALOGRÁFICA

Preparada pelo Serviço de Biblioteca e Informação do Instituto de Física da Universidade de São Paulo

Grande, Helder Luciani Casa
Estudo de cadeias quânticas de Heisenberg
desordenadas
com acoplamentos aperiódicos. São Paulo, 2014.
Tese (Doutorado) - Universidade de São Paulo.
Instituto de Física. Depto. de Física Geral.
Orientador: Prof. Dr. André de Pinho Vieira
Área de Concentração: Física Estatística e
Termodinâmica
Unitermos: 1. Mecânica estatística quântica; 2. Física
da matéria condensada; 3. Mudança de fase.
USP/IF/SBI-037/2014




\section{Agradecimentos}

Agradeço ao meu orientador pela sugestão do trabalho e pela dedicação no projeto ao longo dos últimos anos. Agradeço a Nicolas Laflorencie e Fabien Alet pela colaboração e coorientação neste trabalho, principalmente na parte dos estudos numéricos. Agradeço I. McCulloch por ceder seu algoritmo 1 usado nos cálculos de DMRG. As simulações de Monte Carlo quântico foram feitas usando-se algoritmos [1, 2] do projeto ALPS 2. A parte numérica desse trabalho foi efetuada usando-se recursos computacionais do Laboratório de Física Teórica da Université Paul Sabatier de Toulouse-France. Agradeço a agência Fapesp pelo suporte financeiro.

\footnotetext{
${ }^{1}$ http://physics.uq.edu.au/people/ianmcc/mptoolkit/

${ }^{2}$ http://alps.comp-phys.org
} 



\section{Resumo}

Investigamos as propriedades de baixa temperatura do modelo de Heisenberg unidimensional de spin 1 desordenado, com flutuações geométricas nos acoplamentos induzidas por sequências aperiódicas e determinísticas. Escolhemos duas sequências com propriedades distintas, a sequência de Fibonacci e a sequência 6-3. Nosso objetivo é entender como essas flutuações geométricas modificam a física da fase de Haldane, que corresponde ao estado fundamental da cadeia de spin 1 uniforme. Introduzimos deferentes adaptações do grupo de renormalização de desordem forte (SDRG) de Ma, Dasgupta e Hu, que tem sido amplamente usado no estudo de cadeias de spin aleatórias. Usamos ainda simulações numéricas de Monte Carlo quântico e do grupo de renormalização da matriz densidade para confirmar as previsões do SDRG, assim como estudar as propriedades do estado fundamental, conforme a modulação se torna mais forte. Não encontramos uma transição de fase para a cadeia modulada pela sequência de Fibonacci, enquanto para a cadeia modulada pela sequência 6-3 encontramos uma transição para uma fase sem gap, dominada pela aperiodicidade, similar àquela encontrada na cadeia XXZ de spin 1/2. Mostramos que abordagens que preveem o mesmo comportamento qualitativo na cadeia aleatória de spin 1 podem levar a previsões qualitativamente incompatíveis na cadeia aperiódica 



\begin{abstract}
We investigate the low-temperature properties of the one-dimensional spin-1 Heisenberg model with geometric fluctuations induced by aperiodic but deterministic coupling distributions, involving two parameters. We focus on two aperiodic sequences, the Fibonacci sequence and the 6-3 sequence. Our goal is to understand how these geometric fluctuations modify the physics of the (gapped) Haldane phase, which corresponds to the ground state of the uniform spin-1 chain. We utilize different adaptations of the strong-disorder renormalization-group (SDRG) scheme of Ma, Dasgupta and $\mathrm{Hu}$, widely employed in the study of random spin chains, supplemented by quantum Monte Carlo and density-matrix renormalization-group numerical calculations, to study the nature of the ground state as the coupling modulation is increased. We find no phase transition for the Fibonacci chain, while we show that the 6-3 chain exhibits a phase transition to a gapless, aperiodicitydominated phase similar to that found for the aperiodic spin-1/2 XXZ chain. We show that approaches providing the same qualitative behaviour in the random spin-1 chain may lead to qualitatively incompatible predictions in aperiodic chain.
\end{abstract}





\section{Sumário}

Agradecimentos 1

Resumo 3

$\begin{array}{ll}\text { Abstract } & 5\end{array}$

1 Introdução. $\quad 9$

2 Sequências aperiódicas e o critério de Harris-Luck. 15

2.1 Algumas propriedades das sequências aperiódicas. . . . . . . . . . . . . . 15

2.2 O critério de Harris-Luck para cadeias aperiódicas de spin 1/2 . . . . . . 21

3 Grupo de Renormalizacão de desordem forte para a cadeia de

$\begin{array}{ll}\text { Heisenberg de spins 1/2. } & 27\end{array}$

4 Grupo de renormalização de desordem forte para a cadeia de

$\begin{array}{ll}\text { Heisenberg de spin } 1 . & 33\end{array}$

4.1 Primeira abordagem . . . . . . . . . . . . . . . . . . . . . . 34

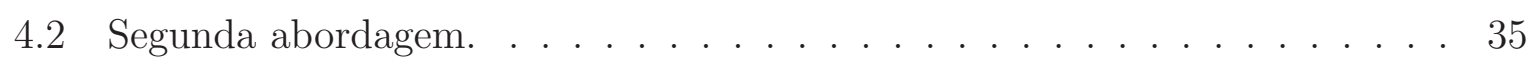

4.3 Terceira abordagem $\ldots \ldots \ldots$. . . . . . . . . . . . . . . . . . . . 39

4.4 Adaptação do método MDH no caso aperiódico . . . . . . . . . . . . . . . 40

5 Cadeia de Fibonacci-Heisenberg de spin 1. 45

5.1 SDRG: Primeira abordagem. . . . . . . . . . . . . . . . . . 46

5.2 SDRG: Segunda abordagem. . . . . . . . . . . . . . . . . . . . . 49

5.3 SDRG: Terceira abordagem . . . . . . . . . . . . . . . . . 50 
6 Cadeia de Heisenberg-6-3 de spin 1.

6.1 SDRG: Primeira abordagem. . . . . . . . . . . . . . . . . 53

6.2 SDRG: Segunda abordagem. . . . . . . . . . . . . . . . . . 55

6.3 SDRG: Terceira abordagem . . . . . . . . . . . . . . . . . . 58

6.4 Relação dinâmica de escala . . . . . . . . . . . . . . . . . . . . . 60

7 Simulacões de Monte Carlo quântico. 63

7.1 O método de Monte Carlo quântico. . . . . . . . . . . . . . . . . . . . . 63

7.1.1 Representacão em série de Tavlor da funcão de particão. . . . . . . . 63

7.1.2 O método de SSE para o modelo de Heisenberg de spin 1/2. . . . . 65

7.2 Simulacões de QMC para a cadeia de Fibonacci-Heisenberg. . . . . . . . . 76

7.3 Simulacõoes de QMC para a cadeia de Heisenberg-6-3. . . . . . . . . . . . . 80

8 Grupo de renormalizacão da matriz densidade. $\quad 83$

8.1 Cálculo do gap e do parâmetro de ordem de corda na cadeia de Fibonacci-

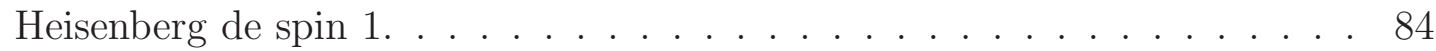

8.2 Cálculo do gap e do parâmetro de ordem de corda na cadeia de Heisenberg$6-3$ de $\operatorname{spin} 1 . \ldots \ldots$. . . . . . . . . . . . . . . . . . 87

9 Método de SDRG com correções variacionais. $\quad 91$

10 Discussão dos resultados e Conclusões. $\quad 97$

$\begin{array}{ll}\text { Apêndices. } & 101\end{array}$

A Resultados da teoria de perturbação em primeira ordem. 101

B Resultados da teoria de perturbação em segunda e terceira ordem. 103

C SDRG: Cálculo do gap para cadeias finitas de Fibonacci-Heisenberg. 105

$\begin{array}{ll}\text { Referências Bibliográficas. } & 107\end{array}$ 


\section{Capítulo 1}

\section{Introdução.}

Neste trabalho estudamos os efeitos da introdução de desordem aperiódica nos acoplamentos da cadeia de Heisenberg de spins 1, visando entender como a desordem pode alterar a natureza do estado fundamental da cadeia uniforme, conhecida como fase de Haldane. A cadeia de spins 1 apresenta um gap entre o estado fundamental e os estados excitados, mostrando uma física profundamente diferente das cadeias de spins $1 / 2$, que não têm um gap separando o estado fundamental dos demais estados excitados. Experimentalmente a cadeia de Heisenberg de spins 1 é realizada de forma quase ideal no composto $\mathrm{Y}_{2} \mathrm{BaNiO}_{5}$ [3], com um gap experimental que concorda bem com as previsões teóricas dadas por $\Delta \sim 0,41 J$ [4]. Cadeias de spins 1 com desordem do tipo quenched (independente do tempo) também foram estudados com a introdução de impurezas não magnéticas nesse mesmo material, $\mathrm{Y}_{2} \mathrm{BaNi}_{1-x} \mathrm{Zn}_{x} \mathrm{O}_{5}$, [5].

As cadeias quânticas de spins representam um excelente laboratório para o estudo, em sistemas de muitos corpos, dos efeitos combinados das flutuações quânticas e da quebra de simetria translacional, introduzida por uma distribuição não homogênea de acoplamentos. Nesse contexto estudamos a cadeia quântica de Heisenberg em uma dimensão, definida pelo hamiltoniano

$$
\mathcal{H}=\sum_{j=1}^{L} J_{j} \mathbf{S}_{j} \cdot \mathbf{S}_{j+1},
$$

onde as constantes $J_{j}>0$ são acoplamentos antiferromagnéticos entre os spins $\mathbf{S}_{i}$ de magnitude $S$, interagindo somente entre primeiros vizinhos. A desordem é introduzida nesse modelo por meio de uma distribuição de acoplamentos $J_{j}>0$ não uniforme. 
Mesmo no limite uniforme $\left(J_{j} \equiv J\right)$, o modelo exibe grande variedade de comportamentos físicos, dependendo se o módulo $S$ dos spins é inteiro ou semi-inteiro. De acordo com a amplamente aceita conjectura de Haldane [6, 4], cadeias com $S$ semi-inteiro têm um espectro de energia sem gap entre o estado fundamental e os primeiros estados excitados, enquanto cadeias com $S$ inteiro apresentam um gap separando o estado fundamental do primeiro estado excitado. Os efeitos mais notáveis ocorrem nos casos $S=\frac{1}{2}$ e $S=1$. A cadeia de spin $1 / 2$ tem um estado fundamental crítico, com correlações decaindo de forma polinomial. Já o estado fundamental do modelo de Heisenberg de spins 1 não é crítico, exibindo decaimento exponencial das correlações. No caso da cadeia de spin 1, o estado fundamental é conhecido como fase de Haldane, e pode ser descrito como um estado de sólido de ligações de valência [7], que exibe uma ordem topológica escondida, revelada pela função de correlação de corda [8, 9], dada por

$$
\left\langle O^{z}(i, j)\right\rangle=\left\langle\Psi_{0}\left|S_{i}^{z} \exp \left(i \pi \sum_{k=i+1}^{j-1} S_{k}^{z}\right) S_{j}^{z}\right| \Psi_{0}\right\rangle,
$$

onde $\left|\Psi_{0}\right\rangle$ é o estado fundamental do modelo de Heisenberg de spin 1. Na fase de Haldane, o limite $|j-i| \rightarrow \infty$ assume um valor não-nulo, assumindo assim o papel de um parâmetro de ordem de corda.

Esse estado fundamental possui ainda graus de liberdade de spin 1/2 livres nas extremidades de uma cadeia finita [10]. De forma simplificada o estado de sólido de ligações valência citado acima pode ser descrito interpretando cada spin 1 como um par de spins $1 / 2$ simetrizados no mesmo sítio. O primeiro spin $1 / 2$ forma um estado de singleto com um spin $1 / 2$ do sítio imediatamente à direita, enquanto o segundo spin $1 / 2$ forma um estado de singleto com um spin $1 / 2$ do sítio imediatamente à esquerda. A ordem topológica presente na fase de Haldane pode ser entendida como a configuração onde, em cada ligação da cadeia, há um singleto formado por spins $1 / 2$ de sítios diferentes. Assim, nas extremidades de uma cadeia finita com condições de contorno abertas, há graus de liberdade de spins $1 / 2$ livres não emparelhados.

A presença ou ausência de um gap no espectro de baixas energias influencia não somente as propriedades termodinâmicas de baixa temperatura, como afeta ainda a estabilidade do estado fundamental do sistema uniforme quando se quebra a simetria translacional (modelo desordenado). No caso mais simples onde se introduz uma dimerização 
(introdução de acoplamentos alternados $J_{\text {par }}$ e $J_{\text {ímpar }}$ ao longo da cadeia), abre-se um gap finito na cadeia de spin $\frac{1}{2}$ mesmo na presença de uma diferença infinitesimal entre $J_{\text {par }}$ e $J_{\text {ímpar }}$, enquanto a fase de Haldane sobrevive à dimerização.

Efeitos devidos a desordem no modelo (quebra de simetria translacional), representados por uma distribuição aleatória de acoplamentos, são ainda mais fortes. No contexto da cadeia de spin $1 / 2$, as mudanças no comportamento físico devido aos efeitos dos acoplamentos aleatórios podem ser estudadas pelo grupo de renormalização de desordem forte (SDRG) introduzido por Ma, Dasgupta e Hu [11, 12, 13. Este método elimina iterativamente os graus de liberdade de altas energias, associados aos pares de spins fortemente acoplados, que pouco contribuem para as propriedades magnéticas de baixas temperaturas, podendo ser dizimados, para dar origem a acoplamentos efetivos mais fracos entre os spins remanescentes.

Vários estudos ao longo dos últimos anos [14, 15, 16, 17, 18, mostraram que, na presença de desordem de qualquer magnitude, o estado fundamental sofre uma transição para uma fase de singleto aleatório, que pode ser visualizada como uma coleção de pares de spins separados por distâncias arbitrárias e acoplados em estados de singletos. Isso é uma consequência, na linguagem do grupo de renormalização, de que a desordem induz um fluxo da distribuição de probabilidades dos acoplamentos efetivos na direção de um ponto fixo de desordem infinita, no qual, em dada escala de energia, há apenas alguns spins fortemente acoplados em estados de singletos, enquanto a grande maioria dos acoplamentos remanescentes são muito fracos. Na fase de singleto aleatório, as propriedades físicas são distintas daquelas observadas na cadeia uniforme, sendo caracterizadas por uma dinâmica ativada, na qual escalas de energia e comprimentos não são relacionados por uma forma polinomial, mas sim por uma forma exponencial. Além disso, no estado fundamental, as correlações são dominadas pelos raros singletos, gerando uma crucial diferença entre comportamentos típicos e médios [15].

Por outro lado, para as cadeias de spin 1, investigações baseadas em extensões do SDRG [19, 20, 21, 22, 23, 24, assim como estudos numéricos 25, 26, 27, 28, 29, 130, mostraram que a fase de Haldane é estável frente a desordem fraca11. Porém para desordem suficientemente forte, uma transição para uma fase de singleto aleatório é observada. Para desordem intermediária foi observado uma fase de Haldane com gap nulo, embora o parâmetro de ordem de corda seja não nulo [26]. Essa fase intermediária está associada a

\footnotetext{
${ }^{1} \mathrm{~A}$ desordem fraca é introduzida no modelo por meio de uma distribuição estreita de acoplamentos.
} 
efeitos de Griffiths [19, 31].

Para as cadeias de spin 1/2, efeitos similares àqueles produzidos pela desordem aleatória podem ser induzidos por desordem aperiódica e determinística. Este tipo de desordem é motivada como um análogo unidimensional dos quase-cristais [32], que são estruturas determinísticas, porém, não periódicas, que apresentam uma simetria proibida pela cristalografia tradicional. As sequências aperiódicas podem ser geradas por uma regra de substituição, como a sequência de Fibonacci, dada por $a \rightarrow a b, b \rightarrow a$. A iteração dessa regra gera uma sequência de letras abaababa ..., para a qual nenhum período pode ser identificado mesmo no limite de uma sequência infinita. Introduzimos a desordem aperiódica nos modelos unidimensionais pela associação da distribuição de letras $a$ e $b$ com a distribuição de acoplamentos $J_{a}$ e $J_{b}$ ao longo da cadeia de spins. Sequências aperiódicas diferentes dão origem a diferentes flutuações geométricas, caracterizadas por um expoente $\omega$, associado a uma lei de potência que descreve o crescimento das flutuações com o crescimento da sequência 2 .

Uma adaptação do SDRG para cadeias do modelo XXZ aperiódicas de spin 1/2 [33, 34] revelou que as propriedades termodinâmicas do estado fundamental são profundamente alteradas pela desordem aperiódica gerada por sequências com $\omega \geq 0$, e um comportamento similar ao da fase de singleto aleatório é observado. Há um comportamento exponencial na relação entre escalas de energia e escala de comprimento, comportamento de escala dinâmico ativado, e uma clara distinção entre comportamentos médios e comportamentos típicos nas funções de correlação entre os spins, neste caso associada com a existência de comprimentos característicos emergentes da combinação de flutuações aperiódicas e quânticas, além do que, em contraste com a fase de singleto aleatório, as correlações podem exibir um estrutura ultra-métrica relacionada com a simetria de inflação da sequência aperiódica.

Neste trabalho, nosso foco é investigar os efeitos da desordem aperiódica sobre as propriedades do estado fundamental da cadeia de Heisenberg de spin 1, conhecido como fase de Haldane. Assim como ocorre no caso de desordem aleatória, esperamos que a fase de Haldane seja estável frente a desordem de modulação fraca (medida pela razão $\left.r=J_{b} / J_{a} \simeq 1\right)$, porém, para modulação forte $(r \rightarrow 0$ ou $r \rightarrow \infty)$ esperamos que a desordem aperiódica induza uma transição de fase, para uma fase dominada pela aperi-

\footnotetext{
${ }^{2}$ Nesse trabalho também utilizamos a sequência $6-3$, definida por $a \rightarrow b a b a a a, b \rightarrow b a a$, que gera flutuações geométricas muito mais fortes que as flutuações da sequência de Fibonacci.
} 
odicidade, muito similar àquela observada na correspondente cadeia aperiódica de spin 1/2. Para isso, obtemos resultados analíticos usando diferentes abordagens do SDRG. Os resultados analíticos são confrontados com resultados numéricos obtidos de simulações de Monte Carlo quântico (QMC) e pelo grupo de renormalização da matriz densidade (DMRG). A primeira abordagem do SDRG é válida somente no limite de modulação forte, e corresponde à generalização direta para variáveis de spin 1 da abordagem proposta por Ma, Dasgupta e Hu [11, 12, 13]. Assim, identifica-se o par, mais geralmente um bloco de spins, acoplado pelo parâmetro mais forte da cadeia, e calculam-se os acoplamentos efetivos entre os spins vizinhos ao bloco dizimado, assumindo-se que os spins do bloco estão congelados em seu estado fundamental. No caso mais simples onde o bloco é composto por apenas um par, o estado fundamental é um singleto, os estados excitados consistem de um tripleto e de um quintupleto, que assumimos contribuírem para o acoplamento efetivo por meio de excitações virtuais.

Como essa abordagem falha para desordem de modulação intermediária [21, várias abordagens alternativas foram propostas [22, 23, 24, 20]. Uma delas, a segunda abordagem utilizada nesse trabalho, consiste em dizimar apenas os estados excitados de mais alta energia, envolvendo a introdução de spins efetivos, e calculando os acoplamentos efetivos entre os spins efetivos e os spins vizinhos, de modo a preservar os níveis de energia mais baixos do bloco renormalizado. Quando o bloco é formado por um par de spins, isto equivale a substituir o par de spins 1 por um par de spins $1 / 2$, conectados pelas mesmas ligações da cadeia original. É conhecido que esse processo não reproduz todos os elementos no subespaço preservado dos estados locais [22, 24] formado por estados de singleto e tripleto. Esse problema pode ser corrigido pela introdução de ligações não frustradas mais longas que as de primeiros vizinhos, o que define a terceira abordagem que utilizamos neste trabalho.

Para desordem aleatória, espera-se que a segunda e a terceira abordagens levem aos mesmo resultados, ao menos qualitativamente. Porém, como mostramos neste trabalho esse não é necessariamente o caso quando estudamos desordem causada por uma distribuição aperiódica de acoplamentos. Argumentamos que a equivalência qualitativa da segunda e terceira abordagens pode ser encontrada somente quando as flutuações geométricas, medidas pelo expoente $\omega$, são suficientemente fortes. Os detalhes desse argumento serão mostrados na conclusão quando introduzirmos a equação (10.1).

Na sequência deste trabalho, mostramos no capítulo 2 conceitos importantes rela- 
cionados com as sequências aperiódicas quando estudamos os efeitos dessa desordem em modelos de física estatística. Após as definições básicas, introduzimos o conceitos de flutuações geométricas e como quantificá-las. Por fim introduzimos o critério de Harris-Luck que estabelece quando as flutuações geométricas induzidas por uma sequência aperiódica são relevantes para modificar o comportamento crítico de um modelo em questão. Com isso justificaremos a escolha das duas sequências utilizadas aqui.

Antes de introduzir as diferentes abordagens utilizadas nesse trabalho mostramos no capítulo 3 uma breve revisão do método introduzido por Ma, Dasgupta e Hu para o modelo de Heisenberg de spins 1/2. As diferentes abordagens do SDRG para cadeias de spin 1 são descritas no capítulo 4. onde mostramos também as necessárias adaptações para lidar com a dizimação de blocos compostos por muitos spins, que são característicos dos modelos aperiódicos.

Nos capítulos 5 e 6 aplicamos as três abordagens para a cadeia de Heisenberg de spin 1 com acoplamentos modulados pelas sequências de Fibonacci e 6-3, as quais respectivamente induzem flutuações geométricas caracterizadas por um expoente $\omega=0$ e $\omega \simeq 0.43$. Portanto, as flutuações geométricas são mais fortes para a segunda sequência.

Os resultados analíticos são confrontados com simulações de Monte Carlo quântico no capítulo 7, e com cálculos numéricos usando o grupo de renormalização da matriz densidade no capítulo 8 ,

Por fim mostramos no capítulo 9 uma diferente adaptação do método de renormalização que não utiliza cálculos perturbativos. Nessa adaptação usaremos métodos variacionais para encontrar os níveis mais baixos de energia que melhor reproduzem o espectro de baixas energias do bloco dizimado em cada passo do grupo de renormalização. Mostraremos alguns resultados preliminares quando aplicamos essa adaptação ao modelo de Ising com campo transverso. Embora promissor, o método SDRG com aproximação local variacional não obteve resultados satisfatórios até o momento. Logo após apresentamos a discussão do resultados e a conclusão do trabalho. Alguns detalhes técnicos são apresentados em apêndices.

Grande parte dos resultados apresentados nessa tese fazem parte do artigo [35], recentemente publicado na Physical Review B. 


\section{Capítulo 2}

\section{Sequências aperiódicas e o critério de Harris-Luck.}

\subsection{Algumas propriedades das sequências aperiódi- cas.}

O interesse em sequências aperiódicas no estudo de modelos de física estatística está relacionado com a descoberta de materiais com estruturas quase-cristalinas [32], que são estruturas determinísticas, porém não periódicas, e que apresentam um simetria proibida pela teoria das redes de Bravais. Essas estruturas podem ser obtidas, em um modelamento matemático, como uma projeção de uma estrutura cristalina periódica em um espaço de dimensão inferior [36].

Análogos unidimensionais dos quase-cristais podem ser obtidos a partir de uma rede bidimensional periódica, projetando-se os pontos de uma seção da rede em um eixo que corta a rede segundo um inclinação irracional. Um exemplo é fornecido pela rede de Fibonacci, mostrada na figura 2.1, cujos pontos consecutivos são separados por duas distâncias possíveis, $a$ e $b$, que formam uma sequência aperiódica. Essa sequência de 
Fibonaccil pode ser produzida pela regra de substituição

$$
\boldsymbol{\sigma}_{F i b}:\left\{\begin{array}{rl}
a & \rightarrow a b \\
b & \rightarrow a
\end{array},\right.
$$

que gera uma sequência infinita de letras pela sucessiva iteração dessa regra em uma sequência inicial, formada somente por uma letra $a$ (ou $b$ ), da seguinte maneira

$$
\begin{aligned}
a & \rightarrow \sigma(a)=a b \rightarrow \\
\rightarrow \sigma^{2}(a) & =\sigma(a) \sigma(b)=a b a \rightarrow \\
\rightarrow \sigma^{3}(a) & =\sigma(a) \sigma(b) \sigma(a)=a b a a b \rightarrow \\
\rightarrow \sigma^{4}(a) & =\sigma(a) \sigma(b) \sigma(a) \sigma(a) \sigma(b)=a b a a b a b a \rightarrow \cdots,
\end{aligned}
$$

onde nenhum período pode ser encontrado [37, 38].

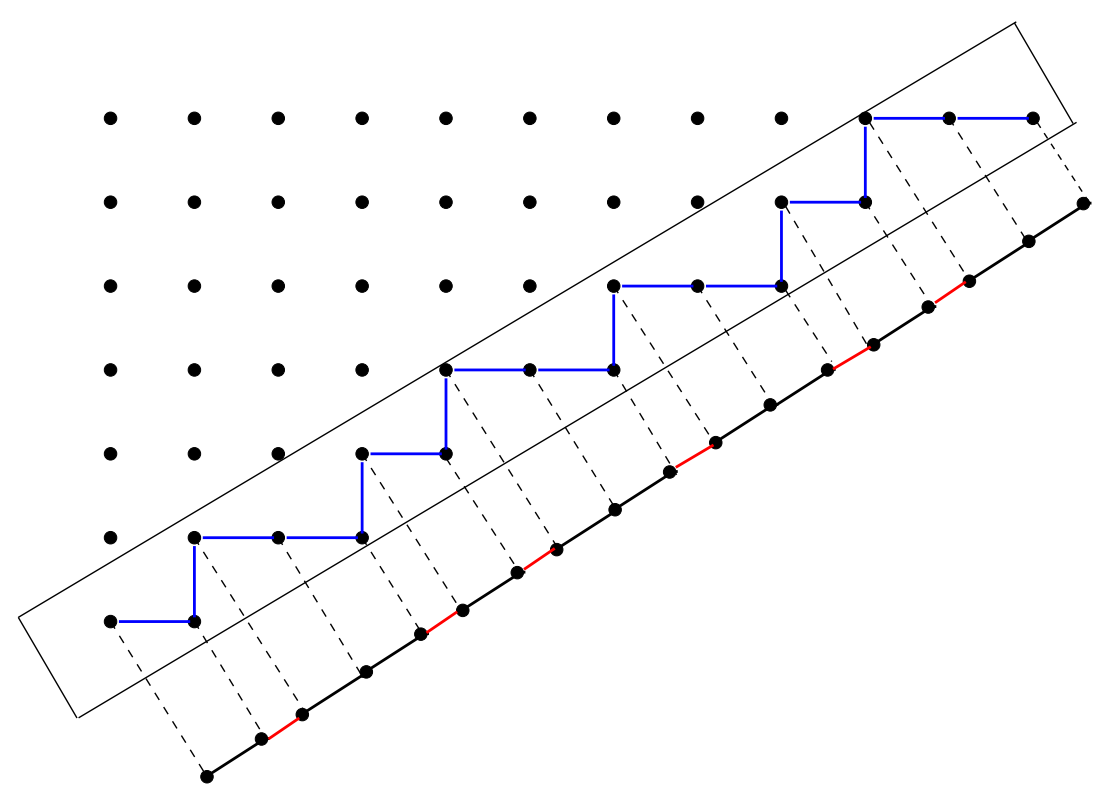

Figura 2.1: Projeção em uma dimensão de uma estrutura periódica bidimensional gerando a sequência de Fibonacci, onde as ligações negras (longas) são letras $a$ e as vermelhas (curtas) letras $b$. O ângulo de inclinação é relacionado com a razão áurea pela expressão $\tan \theta=(1+\sqrt{5}) / 2$.

\footnotetext{
${ }^{1}$ A sequência de Fibonacci recebe esse nome porque o número de letras em cada iteração segue a sequência de números de Fibonacci
} 
Neste trabalho, além da sequência de Fibonacci que acabamos de introduzir, utilizaremos a sequência 6-3, definida como

$$
\sigma_{6-3}:\left\{\begin{aligned}
a & \rightarrow \text { babaaa } \\
b & \rightarrow \text { baa }
\end{aligned}\right.
$$

que do ponto de vista das flutuações geométricas, que serão discutidas a seguir, representa uma sequência com flutuações geométricas mais fortes do que as flutuações da sequência de Fibonacci.

As sequências aperiódicas então são usadas para introduzir flutuações geométricas, ou desordem espacial determinística, nos modelos estatísticos, com o interesse no estudo das alterações do comportamento físico do modelo uniforme, causado por essas flutuações. Pode-se introduzir a desordem aperiódica em um modelo unidimensional de spins distribuindo dois acoplamentos diferentes, digamos $J_{a}$ e $J_{b}$, de acordo com uma determinada sequência aperiódica.

\section{Matriz de substituição e as flutuações geométricas.}

Discutiremos a partir desse ponto algumas propriedades básicas das sequências aperiódicas. Neste trabalho nos interessamos apenas por sequências compostas por duas letras. Podemos assim definir uma regra de substituição genérica do tipo

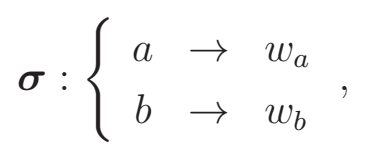

onde $w_{a}$ e $w_{b}$ são palavras compostas pelas letras $a$ e $b$.

Definindo $N_{a}^{(i)}\left(N_{b}^{(i)}\right)$ como o número de letras $a(b)$ em uma sequência de letras depois da $i$-ésima iteração da regra de substituição, podemos também definir a matriz de substituição, a partir da equação de recorrência

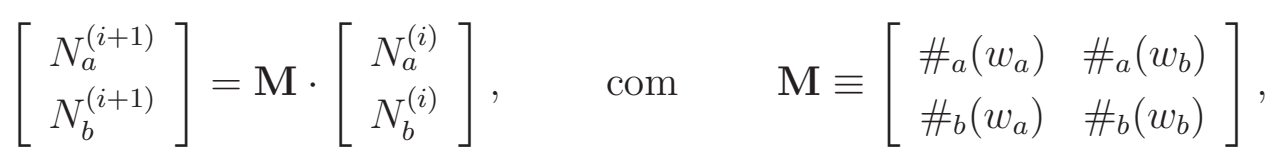

onde $\#_{a}\left(w_{b}\right)$ significa o número de letras a na palavra $w_{b}$. Denotamos o maior (menor) autovalor dessa matriz como $\lambda_{+}\left(\lambda_{-}\right)$. Podemos iterar a equação à esquerda em $2.5 \mathrm{e}$ 
obter o número de letras na $i$-ésima iteração em função do número de letras iniciais

$$
\left[\begin{array}{c}
N_{a}^{(i)} \\
N_{b}^{(i)}
\end{array}\right]=(\mathbf{M})^{i} \cdot\left[\begin{array}{c}
N_{a}^{(0)} \\
N_{b}^{(0)}
\end{array}\right] .
$$

O vetor que representa o número inicial de letras usualmente tem entradas $N_{a}^{(0)}=1$ e $N_{b}^{(0)}=0$. Podemos escrever o vetor que representa os números de letras $a$ e $b$ após $i$ iterações, como um combinação linear dos autovetores da matriz de substituição,

$$
\left[\begin{array}{c}
N_{a}^{(i)} \\
N_{b}^{(i)}
\end{array}\right]=\left(\lambda_{+}\right)^{i} c_{+}|+\rangle+\left(\lambda_{-}\right)^{i} c_{-}|-\rangle,
$$

onde $c_{+}$e $c_{-}$são constantes. No limite assintótico $i \rightarrow \infty$, o termo com o menor autovalor $\left(\lambda_{-}\right)$pode ser desprezado. Assim podemos calcular a frequência das letras $a(b)$ em uma sequência infinita como

$$
f_{a, b}=\lim _{i \rightarrow \infty} \frac{N_{a, b}^{(i)}}{N_{a}^{(i)}+N_{b}^{(i)}}=a_{1,2},
$$

onde $a_{1}, a_{2}$ são as componentes do autovetor $|+\rangle$. A normalização dessas componentes obedece à $a_{1}+a_{2}=1$, para que possamos interpretar esses valores como probabilidades. Ou seja, as componentes do autovetor associado ao maior autovalor determinam as frequências $f_{a}\left(f_{b}\right)$ das letras $a(b)$ em uma sequência infinita.

Também podemos mostrar, a partir da equação (2.7), que o autovalor $\lambda_{+}$representa o fator de escala, ou a razão dos comprimentos, entre duas iterações consecutivas da regra de substituição, no limite assintótico. Para isso precisamos calcular

$$
\lim _{i \rightarrow \infty} \frac{N^{(i+1)}}{N^{(i)}}=\lambda_{+}
$$

onde $N^{(i)}=N_{a}^{(i)}+N_{b}^{(i)}$.

A propriedade de maior interesse em nosso trabalho diz respeito às flutuações geométricas geradas pela sequência aperiódica. Essas flutuações estão ligadas ao menor autovalor $\lambda_{-}$. Uma flutuação geométrica forte significa que, conforme a sequência cresce, mais essa se difere de uma sequência homogênea ou periódica. 
As flutuações geométricas podem ser medidas pela diferença entre o número de letras $a$ em uma sequência após $i$ iterações da regra $\boldsymbol{\sigma}$ e o valor esperado de letras a nessa sequência, dado por $f_{a} N^{(i)}$. Explicitamente,

$$
g_{i}=\left|N_{a}^{(i)}-f_{a} N^{(i)}\right| \sim\left|\lambda_{-}\right|^{i}
$$

Usando $N^{(i)} \sim \lambda_{+}^{i}$, podemos escrever a última equação na forma,

$$
g_{i} \sim\left(N^{(i)}\right)^{\omega_{l}}
$$

definindo assim o expoente de flutuação geométrica,

$$
\omega_{l}=\frac{\ln \left|\lambda_{-}\right|}{\ln \lambda_{+}}
$$

As flutuações geométricas são consideradas ilimitadas, quando o expoente de flutuação geométrica é positivo, $\omega_{l}>0$. No caso oposto, $\omega_{l}<0$, as flutuações geométricas são limitadas, tornando-se menores com o crescimento da sequência, ou seja, a sequência se torna muito próxima de uma sequência periódica [37]. Há também o caso marginal $\omega_{l}=0$.

Repare que denotamos esse expoente como $\omega_{l}$, para diferenciá-lo do expoente citado na introdução $\omega$. O expoente $\omega_{l}$ é uma característica puramente geométrica das sequências aperiódicas, enquanto o expoente $\omega$ leva em conta os possíveis efeitos da flutuação geométrica no comportamento crítico do sistema em estudo. Isso será melhor explicado na seção 2.2 quando o expoente $\omega$ será definido.

\section{Matriz de substituição da sequência de Fibonacci.}

A partir da regra de substituição da sequência de Fibonacci, equação (2.1), e da definição da matriz de substituição (2.5) obtemos

$$
\mathbf{M}_{F i b}=\left[\begin{array}{ll}
1 & 1 \\
1 & 0
\end{array}\right]
$$


com os autovalores e expoente de flutuação geométrica dados por

$$
\lambda_{ \pm}^{F i b}=\frac{1 \pm \sqrt{5}}{2}, \quad \omega_{l}=\frac{\ln \left|\lambda_{-}\right|}{\ln \lambda_{+}}=-1
$$

indicando que as flutuações geométricas são limitadas. Na próxima seção mostraremos que para essa sequência temos o expoente $\omega=0$ quando estudamos a cadeia XX, indicando uma flutuação geométrica marginalmente relevante para alterar o comportamento crítico desse modelo2. Estudos utilizando SDRG indicam flutuações geométricas típicas de $\omega=0$ também para a cadeia XXZ [34].

O autovetor associado com o maior autovalor é dado por

$$
|+\rangle_{F i b}=\frac{1}{1+\lambda_{+}}\left[\begin{array}{c}
\lambda_{+} \\
1
\end{array}\right] \equiv\left[\begin{array}{l}
f_{a} \\
f_{b}
\end{array}\right]
$$

onde $f_{a, b}$ são as frequências das letras $a$ e $b$ em uma sequência de Fibonacci infinita.

\section{Matriz de substituição da sequência 6-3.}

A partir da regra de substituição para a sequência 6-3, equação (2.3), e da definição da matriz de substituição (2.5) obtemos

$$
\mathbf{M}_{6-3}=\left[\begin{array}{ll}
4 & 2 \\
2 & 1
\end{array}\right]
$$

com autovalores e expoente de flutuação geométrica dados por

$$
\lambda_{ \pm}^{6-3}=5,0, \quad \omega_{l}=\frac{\ln \left|\lambda_{-}\right|}{\ln \lambda_{+}}=-\infty
$$

que indica que as flutuações geométricas são limitadas. Na próxima seção mostraremos que para a sequência 6 -3 temos o expoente $\omega=\ln 2 / \ln 5$ quando estudamos a cadeia XX, indicando uma flutuação geométrica relevante para alterar o comportamento crítico desse modelo. Estudos utilizando SDRG indicam flutuações geométricas típicas de um $\omega$ positivo também para a cadeia XXZ [34].

\footnotetext{
${ }^{2} \mathrm{O}$ critério que indica quando uma flutuação geométrica pode alterar o comportamento crítico de um modelo também será introduzido na próxima seção.
} 
O autovetor associado ao maior autovalor é

$$
|+\rangle_{6-3}=\frac{1}{3}\left[\begin{array}{l}
2 \\
1
\end{array}\right] \equiv\left[\begin{array}{c}
f_{a} \\
f_{b}
\end{array}\right] .
$$

onde $f_{a, b}$ são as frequências de letras $a$ e $b$ em uma sequência infinita da sequência 6-3.

\subsection{O critério de Harris-Luck para cadeias aperiódicas de spin $1 / 2$.}

O critério de Harris-Luck 3 estabelece quando flutuações geométricas podem alterar o comportamento crítico de um modelo magnético. Dizemos assim que as flutuações geométricas são relevantes. Segundo esse critério, se as flutuações nos parâmetros locais que controlam a criticalidade no modelo variam com algum comprimento característico na forma $g \sim L^{\omega}$, o comportamento crítico é alterado se o expoente de flutuação geométrica satisfaz a desigualdade

$$
\omega>\omega_{c}=1-\frac{1}{D \nu}
$$

onde $D$ é o número de dimensões ao longo das quais a desordem está distribuída e $\nu$ é o expoente crítico associado ao comprimento de correlação 4 do sistema uniforme subjacente [40]. Quando $\omega<\omega_{c}$ as flutuações geométricas são irrelevantes e não alteram o comportamento crítico. Há ainda o caso marginal $\omega=\omega_{c}$, que podem levar o modelo a um comportamento crítico não universal.

\section{Demonstração heurística do critério de Harris-Luck.}

Esse critério pode ser estabelecido estudando-se como a desordem, nesse caso as flutuações geométricas aperiódicas, altera localmente a distância à criticalidade do modelo sem desordem, modificando assim o comportamento crítico. Para isso considere o modelo de

\footnotetext{
${ }^{3} \mathrm{O}$ critério de Harris foi primeiramente estabelecido para determinar se a desordem aleatória poderia alterar o comportamento crítico de um modelo magnético [39], sendo posteriormente adaptado para o caso de desordem aperiódica [40].

${ }^{4}$ Para o modelo XXZ o expoente $\nu$ varia continuamente de 1 no limite do modelo XX até $2 / 3$ no limite do modelo de Heisenberg [41.
} 
Ising, definido em uma rede $D$-dimensional, com uma iteração não uniforme

$$
\mathcal{H}_{\text {Ising }}=-\sum_{\langle i, j\rangle} J_{i, j} \sigma_{i} \sigma_{j}
$$

onde $J_{i, j}>0$, modelo ferromagnético.

No caso uniforme $J_{i, j} \equiv J_{0}$, o modelo apresenta uma transição de fase, de uma fase desordenada em altas temperaturas, para uma fase ordenada em baixas temperaturas. A transição ocorre em um temperatura crítica $T_{c} \propto J_{0}$.

Nas proximidades do ponto crítico o comprimento de correlação diverge como

$$
\xi \sim|t|^{-\nu}
$$

onde $\nu$ é o expoente crítico associado ao comprimento de correlação. A distância à criticalidade é definida como $t=\left(T-T_{c}\right) / T_{c}$.

O comprimento de correlação define um volume $\xi^{D}$ onde os spins estão fortemente correlacionados. Nesse volume a flutuação geométrica se comporta como $\xi^{D \omega}$. Pode-se quantificar as alterações na distância à criticalidade causadas pelas flutuações geométricas, calculando-se a flutuação geométrica média sobre o volume definido pelo comprimento de correlação, dada por

$$
\delta t \sim \frac{\xi^{D \omega}}{\xi}=|t|^{D \nu(1-\omega)}
$$

onde usamos $\xi \sim|t|^{-\nu}$. Assim $\delta t$ mostra o quanto a distância à criticalidade se altera devido à desordem nos parâmetros $\left\{J_{i, j}\right\}$, devendo ser comparado com $t$ do modelo uniforme. Para essa comparação escrevemos

$$
\frac{\delta t}{|t|} \sim|t|^{D \nu(1-\omega)-1} .
$$

Temos $\delta t \gg t$, desordem relevante, quando o expoente da última equação é negativo. O caso contrário de desordem irrelevante, $\delta t \ll t$, ocorre quando o expoente é positivo. Assim encontramos um valor crítico de $\omega$ dado por

$$
\omega_{c}=1-\frac{1}{D \nu}
$$

Salientamos ainda que esse critério é perturbativo, fornecendo informações apenas 
para desordem de pequena intensidade.

\section{Distância à criticalidade para o modelo $\mathrm{XX}$.}

Repare que denotamos o expoente de flutuação geométrico do critério de Harris-Luck $(\omega)$, equação (2.19), de forma diferente do expoente da equação (2.12) $\left(\omega_{l}\right)$. De fato, esses expoentes não são necessariamente os mesmos de acordo com o modelo magnético em questão. Mais precisamente precisamos conhecer como se comporta o parâmetro que define a distância à criticalidade do modelo como função da desordem.

Para essa discussão, considere o hamiltoniano do modelo XXZ, para spins $1 / 2$, definido em uma cadeia com $L$ sítios, dado por

$$
\mathcal{H}=\sum_{j=1}^{L} J_{j}\left(s_{j}^{x} s_{j+1}^{x}+s_{j}^{y} s_{j+1}^{y}+\Lambda_{j} s_{j}^{z} s_{j+1}^{z}\right),
$$

onde $J_{j}>0$, com a anisotropia dependente do sítio obedecendo à $0 \leqslant \Lambda_{j} \leqslant 1$. No limite $\Lambda_{j}=1$ temos o modelo de Heisenberg, e no limite oposto, $\Lambda_{j}=0$, temos o modelo XX.

Seguimos agora com uma análise feita por Hermisson [42, para o modelo XX com presença de desordem aperiódica, onde foi formulado como calcular o expoente de flutuação geométrico que deve ser usado no critério de Harris-Luck.

O comportamento crítico do modelo XX é susceptível a dimerização, isto é, a introdução de parâmetros $J_{\text {par }}$ e $J_{\text {ímpar }}$ em posições alternadas. Essa dimerização induz a cadeia a uma transição para uma fase dimerizada, com gap, e portanto não crítica. Mais precisamente, ocorre uma transição de fases quântica à temperatura zero entre duas fases dimerizadas quando 5

$$
\delta=\ln \left(\frac{J_{\text {ímpar }}}{J_{\text {par }}}\right)=0 .
$$

Assim o parâmetro local que mede a distância à criticalidade pode ser escrito como $\delta_{j}=\ln \left(J_{2 j-1} / J_{2 j}\right)$. Portanto devemos considerar flutuações geométricas, e uma matriz de substituição, que dependam dos pares de letras para estudar as flutuações locais de $\delta_{j}$.

Segundo Hermisson isso é feito iterando a regra original até obtermos duas palavras com a mesma paridade e identificando todos os tipos de pares presentes. Como exem-

\footnotetext{
${ }^{5}$ Essa condição é um caso especial da condição geral para uma transição de fase quando há uma dimerização média dada por $\delta=\overline{\ln J_{2 j-1}}-\overline{\ln J_{2 j}}=0$.
} 
plo escolhemos novamente a sequência de Fibonacci. Após iterarmos a regra três vezes obtemos

$$
\boldsymbol{\sigma}_{f b}^{3}:\left\{\begin{aligned}
a & \rightarrow a b a a b \\
b & \rightarrow a b a
\end{aligned}\right.
$$

A regra de substituição de pares é dada então por

$$
\boldsymbol{\sigma}_{f b}^{(2)}:\left\{\begin{aligned}
a a & \rightarrow(a b)(a a)(b a)(b a)(a b) \\
a b & \rightarrow(a b)(a a)(b a)(b a) \\
b a & \rightarrow(a b)(a a)(b a)(a b)
\end{aligned}\right.
$$

repare que o par bb não ocorre na sequência. Agora a matriz de substituição de pares é definida por

$$
\mathbf{M}^{(2)}=\left[\begin{array}{llll}
\#_{a a}\left(w_{a a}\right) & \#_{a a}\left(w_{a b}\right) & \#_{a a}\left(w_{b a}\right) & \#_{a a}\left(w_{b b}\right) \\
\#_{a b}\left(w_{a a}\right) & \#_{a b}\left(w_{a b}\right) & \#_{a b}\left(w_{b a}\right) & \#_{a b}\left(w_{b b}\right) \\
\#_{b a}\left(w_{a a}\right) & \#_{b a}\left(w_{a b}\right) & \#_{b a}\left(w_{b a}\right) & \#_{b a}\left(w_{b b}\right) \\
\#_{b b}\left(w_{a a}\right) & \#_{b b}\left(w_{a b}\right) & \#_{b b}\left(w_{b a}\right) & \#_{b b}\left(w_{b b}\right)
\end{array}\right],
$$

o que em nosso caso resulta em

$$
\mathbf{M}^{(2)}=\left[\begin{array}{lll}
1 & 1 & 1 \\
2 & 1 & 2 \\
2 & 2 & 1
\end{array}\right]
$$

Os autovalores dessa nova matriz são $\lambda_{1}=-1$ e $\lambda_{2,3}=2 \mp \sqrt{5}$, que gera um expoente de flutuação geométrica

$$
\omega(\text { Fibonacci }) \equiv \frac{\ln \left|\lambda_{1}\right|}{\ln \lambda_{2}}=0,
$$

portanto, embora as flutuações geométricas geradas pela sequência de Fibonacci sejam limitadas, para a cadeia XX de $\operatorname{spin} 1 / 2, \operatorname{com} \nu=1$, que gera um expoente crítico $\omega_{c}=0$, essas flutuações são marginalmente relevantes e podem alterar o comportamento crítico da cadeia.

A sequência 6-3 gera flutuações relevantes na cadeia XX, porém a formulação que acabamos de ilustrar não se aplica a essa sequência, pois a palavras geradas para cada 
letra nunca têm a mesma paridade. Porém, ainda é possível encontrar uma matriz de substituição e estudar as flutuações em $\delta_{j}$ a partir de segmentos mínimos, como também feito por Hermisson [42, que encontrou o valor do expoente de flutuação geométrica

$$
\omega(\text { sequência } 6-3)=\frac{\ln 2}{\ln 5} \text {. }
$$

Para o caso mais geral do modelo XXZ, e para o limite de Heisenberg $\left(\Lambda_{j} \equiv 1\right)$, não há uma formulação exata para avaliar as flutuações geométricas, análogas as quantidades $\delta_{j}$ definidas para a cadeia XX. No entanto, trabalhos que adaptaram o SDRG para o caso aperiódico [33, 34] verificaram que as propriedades termodinâmicas das cadeias XXZ e de Heisenberg, respondem a introdução de desordem aperiódica essencialmente da mesma forma como responde a cadeia XX, de acordo com o valor de $\omega$ da cadeia XX, ao menos para $\omega>0$. Em todo este trabalho, quando mencionado o expoente de flutuação geométrica $\omega$ de uma dada sequência aperiódica, referimos-nos ao expoente calculado para a cadeia XX calculado por Hermisson.

O critério de Harris-Luck não fornece previsões para a cadeia de Heisenberg de spin 1, pois a fase de Haldane não é crítica. Porém espera-se que as flutuações geométricas geradas pelas sequências de Fibonacci e 6-3 perturbem a fase de Haldane com intensidades diferentes. Isso justifica a escolha das sequências aperiódicas utilizadas neste trabalho. 



\section{Capítulo 3}

\section{Grupo de Renormalização de desordem forte para a cadeia de Heisenberg de spins 1/2.}

O grupo de renormalização de desordem forte proposto por Ma, Dasgupta e Hu consiste na dizimação iterativa do parâmetro de energia mais forte - usualmente um acoplamento ligando dois spins vizinhos - e a sua substituição por um parâmetro efetivo calculado por teoria de perturbação, para assim eliminar os graus de liberdade de mais alta energia presentes no sistema. O novo parâmetro efetivo é sempre menor do que aquele que foi dizimado. Após a primeira dizimação, esse processo é repetido com o próximo parâmetro mais forte na cadeia e assim por diante. No limite assintótico, após um número muito grande de iterações, a hamiltoniana efetiva gerada por esse método deve descrever muito bem o comportamento de baixa energia (baixas temperaturas) do sistema.

O método foi introduzido primeiramente para estudar a cadeia de Heisenberg de spin

$\frac{1}{2}$ com acoplamentos distribuídos de forma aleatória [11, 12, 13] sendo bem sucedido no estudo das propriedades termodinâmicas do estado fundamental da cadeia, o qual foi chamado de fase de singleto aleatório [15]. O primeiro passo do método consiste em encontrar o acoplamento mais forte presente na cadeia, denotado aqui por $J_{0}$. Assumindo que a distribuição de acoplamentos é suficientemente larga, em baixas temperaturas $(T \ll$ $J_{0}$ em unidades apropriadas), podemos supor que o par de spins conectado por $J_{0}$ está congelado no estado fundamental local (estado de singleto), podendo ser eliminado do 
sistema. As excitações virtuais do par dão origem a uma ligação efetiva que acopla os vizinhos desse par, como ilustra a figura 3.1 .

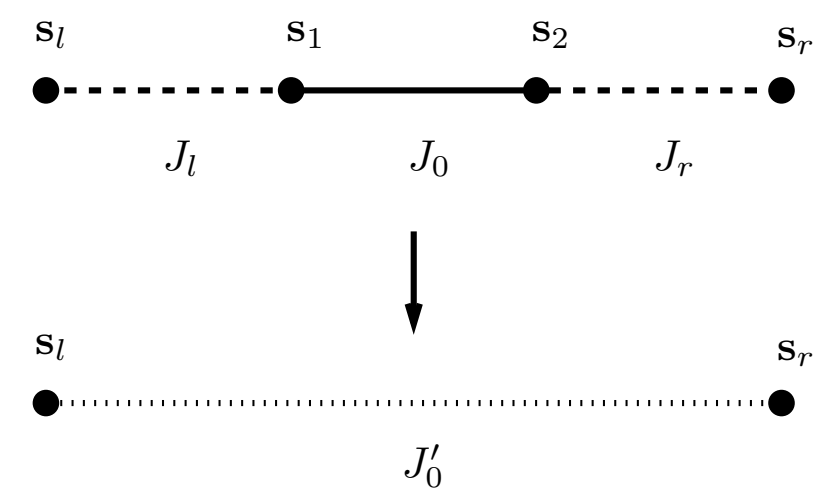

Figura 3.1: Procedimento de dizimação para um par de spins $S=\frac{1}{2}$.

Se assumimos que os acoplamentos vizinhos $J_{l}$ e $J_{r}$ são muito mais fracos que $J_{0}$, podemos calcular o acoplamento efetivo via teoria de perturbação. Nesse caso tratamos as interações entre o par e o resto da cadeia, ligações entre o par e os primeiros vizinhos $\mathbf{s}_{l}$ e $\mathbf{s}_{r}$, como uma perturbação sobre o estado fundamental do par central. Podemos escrever a hamiltoniana local como

$$
\begin{array}{ll}
h=h_{0}+h_{1}, \quad \text { com } \quad & h_{0}=J_{0} \mathbf{s}_{1} \cdot \mathbf{s}_{2}, \\
& h_{1}=J_{l} \mathbf{s}_{l} \cdot \mathbf{s}_{1}+J_{r} \mathbf{s}_{2} \cdot \mathbf{s}_{r},
\end{array}
$$

onde $h_{1}$ representa a perturbação sobre os estados do par $\mathbf{s}_{1}$ e $\mathbf{s}_{2}$ associado a $h_{0}$ (neste texto operadores de spin $\frac{1}{2}$ são representados por letras minúsculas). Os autoestados de $h_{0}$ são um singleto,

$$
\left|\Phi_{0}\right\rangle=\frac{1}{\sqrt{2}}(|+-\rangle-|-+\rangle),
$$

com energia $E_{0}=-\frac{3}{4} J_{0}$, e um tripleto,

$$
\begin{aligned}
\left|\Phi_{1}^{+}\right\rangle & =|++\rangle, \\
\left|\Phi_{1}^{0}\right\rangle & =\frac{1}{\sqrt{2}}(|+-\rangle+|-+\rangle), \\
\left|\Phi_{1}^{-}\right\rangle & =|--\rangle,
\end{aligned}
$$


com energia $E_{1}=\frac{1}{4} J_{0}$. Se assumimos que $h_{0}$ define a escala de energia $\Delta$ do sistema, podemos estimar que $\Delta=E_{1}-E_{0}$, e em baixas temperaturas $\left(k_{b} T \ll \Delta\right)$ o par $\mathbf{s}_{1}$ e $\mathbf{s}_{2}$ está efetivamente congelado no seu estado fundamental.

Até segunda ordem em teoria de perturbação, a hamiltoniana efetiva pode ser escrita como

$$
h^{\mathrm{eff}}=\left\langle\Phi_{0}\left|h_{1}\right| \Phi_{0}\right\rangle+\sum_{i} \frac{\left|\left\langle\Phi_{0}\left|h_{1}\right| \Phi_{1}^{i}\right\rangle\right|^{2}}{E_{0}-E_{1}}=E^{\prime}+J_{0}^{\prime} \mathbf{s}_{l} \cdot \mathbf{s}_{r}
$$

onde a somamos sobre $i \in\{+, 0,-\}$.

Os parâmetros efetivos são dados por

$$
E^{\prime}=-\frac{3}{4} J_{0}-\frac{3}{16} \frac{\left(J_{l}^{2}+J_{r}^{2}\right)}{J_{0}} \quad \text { e } \quad J_{0}^{\prime}=\frac{1}{2} \frac{J_{l} J_{r}}{J_{0}}
$$

onde $E^{\prime}$ representa a correção na energia do estado fundamental de $h$, e $J_{0}^{\prime}$ é o acoplamento efetivo entre os spins $\mathbf{s}_{l}$ e $\mathbf{s}_{r}$. Note que a ligação efetiva $J_{0}^{\prime}$ é sempre menor do que a ligação $J_{0}$, de modo que a escala de energia é consistentemente reduzida.

A iteração da regra de dizimação descrita acima gera uma distribuição efetiva de probabilidades [15] que fica cada vez mais larga com a iteração, sugerindo que assintoticamente os resultados do grupo de renormalização são exatos, pois isso fortalece a hipótese que valida o uso da teoria de perturbação. De fato, o ponto fixo da distribuição dos acoplamentos efetivos tem variância infinita, sendo chamado de ponto fixo de desordem infinita. Isso sempre ocorre independentemente da largura inicial da distribuição de probabilidades. Essa distribuição é fortemente concentrada em $\tilde{J}=0$, e tem a forma de uma lei de potência [11, 12, 15], dada por

$$
P(\tilde{J}, \Delta)=\frac{\alpha}{\Delta}\left(\frac{\Delta}{\tilde{J}}\right)^{1-\alpha} \theta(\Delta-\tilde{J}), \quad \text { onde } \quad \alpha=-1 / \ln \Delta,
$$

onde $\Delta$ é a escala de energia. Essa última equação representa o ponto fixo de desordem infinita para a equação de evolução da distribuição de probabilidades.

No regime assintótico, após um grande número de iterações, a cadeia é formada por pares de spins acoplados em estados de singletos, separados entre si por distâncias arbitrariamente grandes, figura 3.2. Em geral o acoplamento entre os spins que formam o 
singleto é tão mais fraco quanto maior for a distância entre eles. Essa fase é chamada de fase de singleto aleatório e a distribuição assintótica de energias e comprimentos dos singletos é independente da distribuição de probabilidades dos acoplamentos inicial [15].

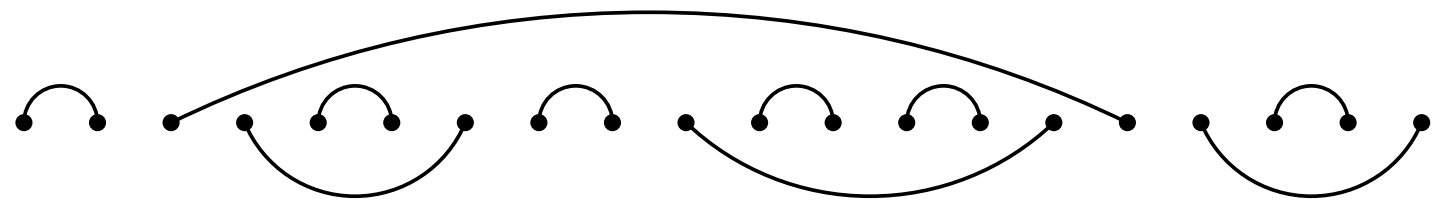

Figura 3.2: Ilustração do estado fundamental da cadeia de spin $1 / 2$ desordenada. Chamada de fase de singleto aleatório, é formada por singletos de spins, que nunca se cruzam, separados por distâncias arbitrárias. Em geral, as ligações longas são mais fracas que as curtas.

A relação entre escalas de energia e comprimento tem uma forma exponencial dada por $\Delta \sim \exp (-c \sqrt{L})$, onde $c$ é uma constante, que mostra um comportamento de escala dinâmico ativado. Aqui a escala de comprimento, em uma dada escala de energia, é dado pelo comprimento dos singletos formados nessa escala.

A susceptibilidade magnética pode ser calculada na fase de singleto aleatório como $\chi(T) \sim n(\Delta) / T$, onde $n(\Delta)$ é o numero de spins não dizimados em uma escala de energia tal que $\Delta \sim T$. Nesse cálculo considera-se que os pares de spin dizimados em escalas de energia maiores que $\Delta$ não contribuem para a susceptibilidade, enquanto aqueles ainda não dizimados se comportam como spins livres e contribuem com um termo de Curie $(\sim 1 / T)$ para a susceptibilidade. Fisher [15] calculou o número de spins remanescentes como sendo $n(\Delta) \sim 1 /[\ln (1 / \Delta)]^{2}$, resultando em uma susceptibilidade dada por

$$
\chi(T) \sim \frac{1}{T[\ln (1 / T)]^{2}}
$$

As correlações na fase de singleto aleatório são profundamente diferentes daquelas no sistema uniforme. Como no estado fundamental, em dada escala de energia, há raros singletos separados por distâncias arbitrárias, e muitos spins fracamente acoplados, que serão dizimados em escalas de energia mais baixas, as correlações médias são diferentes das correlações típica 11 entre os spins. Enquanto a correlação típica entre spins decai exponencialmente, a correlação média é dominada pelos raros singletos fortemente acoplados e decai como um lei de potência $\sim 1 / L^{2}$.

\footnotetext{
${ }^{1}$ Por correlações típicas nos referimos às correlações estatisticamente mais numerosas.
} 


\section{Adaptação do SDRG para o caso de desordem aperiódica.}

Discutimos brevemente a adaptação do SDRG para o caso de desordem aperiódica [33, 34]. Quando a desordem aperiódica é introduzida pela distribuição de apenas dois acoplamentos $J_{a}$ e $J_{b}$, seguindo uma sequência aperiódica de duas letras, surgem blocos de spins conectados por um mesmo acoplamento. Quando esse acoplamento é o mais forte da cadeia, este deve ser dizimado.

Para a cadeia de spin $1 / 2$ há dois casos a serem considerados. O primeiro quando o bloco é formado por um número par de spins. O estado fundamental é sempre dado por um estado de singleto, e a dizimação dos estados excitados gera uma ligação efetiva entre os vizinhos à direita e à esquerda do bloco, situação semelhante à mostrada na figura 3.1 . A formulação da teoria de perturbação, para o cálculo do parâmetro efetivo, é similar à mostrada na equação (3.4), com $h_{0}$ contendo todos os spins do bloco dizimado, e a soma cobrindo todos os estados excitados do bloco.

No segundo caso, quando o bloco contém um número ímpar de spins, o estado fundamental é duplamente degenerado. Como queremos preservar esse estado fundamental, podemos introduzir um spins $1 / 2$ efetivo substituindo o bloco, como ilustrado na figura 3.3. Detalhes de como calcular os parâmetros efetivos $J_{l}^{\prime}$ e $J_{r}^{\prime}$, via cálculos de perturbação em primeira ordem, podem ser encontrados na referência [34].

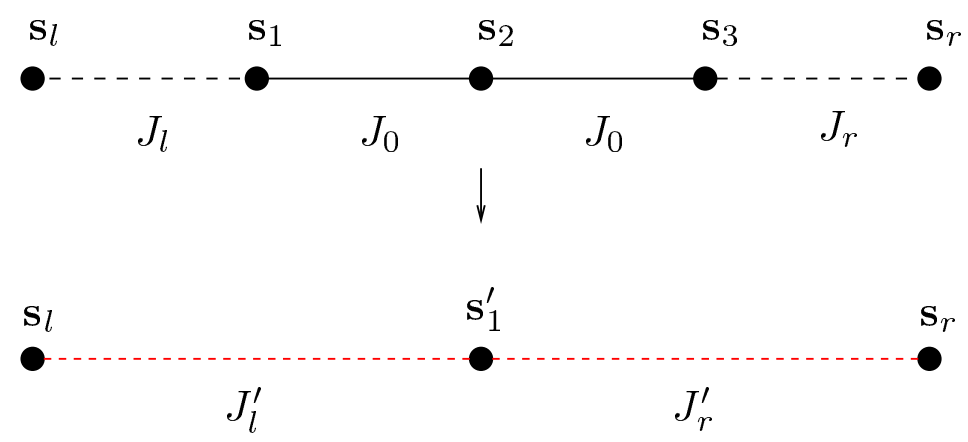

Figura 3.3: Procedimento de dizimação de um bloco formado por três spins $S=\frac{1}{2}$. O estado fundamental é duplamente degenerado, e é preservado pela introdução de um spin efetivo $1 / 2$.

O SDRG adaptado ao caso aperiódico foi utilizado por Vieira [33, 34, para o estudo de cadeias XXZ de spin 1/2, com desordem aperiódica gerada por sequências como Fibonacci e 6-3, além de várias outras. Efeitos muito similares aos causados pela desordem aleatória 
foram observados para desordem aperiódica com expoente $\omega \geqslant 0$. Para essas sequências, o estado fundamental é dominado pelas flutuações geométricas aperiódicas e é chamado de fase de singleto aperiódico. Nessa fase, em dada escala de energia, singletos acoplados por ligações efetivas fortes (digamos $J_{b}^{\prime}$ ) são distribuídos de forma aperiódica, ou seja, há uma distribuição aperiódica de acoplamentos efetivos $J_{a}^{\prime}$ e $J_{b}^{\prime}$. Em escalas de energia mais baixas, os singletos são dizimados dando origem a novas ligações efetivas que conectam os spins remanescentes, novamente distribuídos de forma aperiódica. Sequências que geram spins efetivos também foram encontradas [33, 34].

Para as sequências utilizadas nesta tese, o estudo das equações de recorrência para os parâmetros efetivos $J_{a}^{\prime}$ e $J_{b}^{\prime}$ mostra que a desordem, dada pela razão efetiva $r^{\prime}=J_{b}^{\prime} / J_{a}^{\prime}$, aumenta com a iteração do SDRG. Esse é o ponto fixo de desordem infinita aperiódico, mostrando que o estado fundamental é formado por singletos fortemente acoplados, e quase independentes do resto da cadeia.

O comportamento de escala dinâmico para a cadeias com acoplamentos modulados pela sequência 6-3, que tem flutuações geométricas fortes $\omega>0$, mostra um comportamento exponencial na forma $\Delta \sim \exp \left(L^{-\omega}\right)$. Enquanto para a cadeia modulada pela sequência de Fibonacci a relação de escala dinâmica mostra uma dependência exponencial mais fraca.

Grandezas termodinâmicas como a susceptibilidade magnética ou calor específico, podem ser calculadas usando a mesma aproximação usada por Fisher [15], ou podem ainda ser calculadas de maneira mais eficiente, por uma aproximação chamada de aproximação de pares independentes que também será usada nesse trabalho no capítulo 7 . Essas grandezas termodinâmicas apresentam oscilações características das sequências aperiódicas, com máximos localizados nas escalas de energia de cada dizimação. Resultados numéricos mostraram boa concordância com os resultados providos pelo SDRG [34].

As correlações entre spins do estado fundamental das cadeias aperiódicas também mostram comportamentos típicos e médios diferentes. Pelo fato da distribuição de ligações ser fixa2, há pares de singletos, fortemente correlacionados, formados entre spins conectados pelas sucessivas ligações efetivas $J_{b}^{\prime}$ produzidas a medida que se reduz a escala de energia. As correlações entre os demais pares de spins são muito mais fracas.

\footnotetext{
${ }^{2}$ Após a dizimação das ligações originais, as ligações efetivas em geral seguem a mesma sequência aperiódica, ou alguma sequência similar a essa sequência.
} 


\section{Capítulo 4}

\section{Grupo de renormalização de desordem forte para a cadeia de Heisenberg de spin 1.}

Neste capítulo discutiremos as três diferentes abordagens que podem ser usadas quando adaptamos o SDRG para cadeias de spin 1 [22, 23, 24]. As diferentes abordagens surgem da diferença entre os espectros de um par de spins $1 / 2$ e um par de spins 1, assim como da procura por uma regra de dizimação que consistentemente reduza a escala de energia. Abordagens diferentes das expostas aqui também foram consideradas em outros trabalhos [19, 20, 43].

No caso de uma cadeia de spins 1 a hamiltoniana do par de spins acoplados por $J_{0}$,

$$
h_{0}=J_{0} \mathbf{S}_{1} \cdot \mathbf{S}_{2}=\frac{J_{0}}{2}\left[\left(\mathbf{S}_{1}+\mathbf{S}_{2}\right)^{2}-4\right] \text {, }
$$

tem três níveis de energia (um nível a mais que o caso de spins 1/2), com energias dadas por

$$
e_{s}=\frac{J_{0}}{2}[s(s+1)-4]
$$

em que $s=0,1,2$, e têm degenerescência $(2 s+1): e_{0}=-2 J_{0}$ representa o singleto, $e_{1}=-J_{0}$ representa o tripleto e $e_{2}=J_{0}$ representa o quintupleto.

Com um nível a mais surge a possibilidade de descartarmos todos os estados excitados do par conectado por $J_{0}$, ou descartarmos apenas o estado excitado de mais alta energia. 


\section{Grupo de renormalização de desordem forte para a cadeia de}

A primeira opção dá origem à primeira abordagem descrita na próxima seção, e como essa abordagem não é necessariamente consistente, eventualmente falhando em reduzir sistematicamente a escala de energia do problema, a utilização da segunda opção dá origem a duas abordagens diferentes que envolvem a substituição do par de spins 1 por um par de spins $1 / 2$.

\subsection{Primeira abordagem.}

Esta abordagem é a adaptação direta dos cálculos feitos no capítulo anterior para o par de spins $1 / 2$, porém agora o par é constituído por spins 1 , e eliminamos todos os estados excitados, mantendo apenas o estado de singleto. A hamiltoniana local é definida por

$$
\begin{aligned}
h & =h_{0}+h_{1}, \\
h_{0} & =J_{0} \mathbf{S}_{1} \cdot \mathbf{S}_{2}, \\
h_{1} & =J_{l} \mathbf{S}_{l} \cdot \mathbf{S}_{1}+J_{r} \mathbf{S}_{2} \cdot \mathbf{S}_{r},
\end{aligned}
$$

onde consideramos $h_{1}$ como uma perturbação sobre $h_{0}$ (representamos os operadores de spin 1 sempre com letras maiúsculas). Os níveis de energia de $h_{0}$ são um singleto com energia $E_{0}=-2 J_{0}$, um tripleto, com energia $E_{1}=-J_{0}$ e um quintupleto com energia $E_{2}=J_{0}$. Quando descartamos todos os estados excitados a escala local de energia é definida por $\Delta=E_{1}-E_{0}=J_{0}$, que é a diferença entre o estado fundamental e o primeiro estado excitado eliminado.

Aplicando a teoria de perturbação de segunda ordem na hamiltoniana acima, somando sobre todos os estados excitados de $h_{0}$, como feito na equação (3.4), o acoplamento efetivo entre $\mathbf{S}_{l}$ e $\mathbf{S}_{r}$ é dado por

$$
J_{0}^{\prime}=\frac{4}{3} \frac{J_{l} J_{r}}{J_{0}}
$$

que não é necessariamente consistente, pois as condições $J_{l}<J_{0}$ e $J_{r}<J_{0}$ não são suficientes para garantir que $J_{0}^{\prime}<J_{0}$. Porém este resultado deve ser válido para uma distribuição larga de acoplamentos quando pode-se assegurar que $J_{l}, J_{r} \ll J_{0}$.

Como discutiremos a seguir, a procura por uma regra de dizimação que seja autoconsistente quando a regra logo acima falha, dá origem a duas novas abordagens, nas quais o par de spins 1 é substituído por um par de spins $1 / 2$. 
Quando tratarmos o caso de desordem aperiódica, será necessário lidar com blocos de spins conectados por um mesmo acoplamento, que eventualmente pode definir o maior gap da cadeia. As adaptações necessárias ao SDRG serão estudadas na seção 4.4. Nessa seção calculamos o gap entre o estado fundamental e o primeiro estado excitado eliminado $(\Delta)$, para cada bloco encontrado na cadeia aperiódica. Esses dados são resumidos na tabela 4.2, onde mostramos também a configuração efetiva do bloco renormalizado. Por simplicidade, sempre evitaremos a renormalização de blocos com muitos spins.

\subsection{Segunda abordagem.}

A segunda abordagem que discutiremos foi usada por Monthus et al. [23, 24] no estudo de cadeias de spin 1 com acoplamentos distribuídos de forma aleatória. A idéia é descartar apenas o estado excitado de mais alta energia, o quintupleto, ao invés de descartar todos os estados excitados. Assim preservamos os estados de singleto e tripleto. Pode-se fazer isso substituindo-se o par de spins $1 \mathbf{S}_{1}$ e $\mathbf{S}_{2}$ por um par de spins $1 / 2$ efetivos $\mathbf{s}_{1}^{\prime}$ e $\mathbf{s}_{2}^{\prime}$, também conectados por $J_{0}$, já que em ambos os casos o valor do gap para o primeiro estado excitado é dado por $J_{0}$, reproduzindo assim o gap de menor energia de $h_{0}$. Com essa idéia podemos escrever a hamiltoniana efetiva como

$$
h_{0}^{\mathrm{eff}}=-\frac{5}{4} J_{0}+J_{0} \mathbf{s}_{1}^{\prime} \cdot \mathbf{s}_{2}^{\prime}
$$

Note que a constante $-5 J_{0} / 4$ é usada para ajustar os estados de $h_{0}^{\text {eff }}$ e $h_{0}$ na equação (4.3).

A hamiltoniana local é então substituída por uma hamiltoniana efetiva envolvendo os spins $\mathbf{S}_{l}, \mathbf{s}_{1}^{\prime}, \mathbf{s}_{2}^{\prime}$, e $\mathbf{S}_{r}$

$$
h^{\mathrm{eff}}=h_{0}^{\mathrm{eff}}+h_{1}^{\mathrm{eff}}
$$

como mostramos na figura 4.1. Resta a questão de como determinar o termo perturbativo $h_{1}^{\text {eff }}$, que representa a conexão do par de spin $1 / 2 \mathrm{com}$ a parte remanescente da cadeia. Ao exigirmos que, em primeira ordem de teoria de perturbação, ambos $h_{1}^{\text {eff }}$ e $h_{1}$ na equação (4.3) resultem nos mesmos elementos de matriz dentro de seus respectivos subespaços de singleto e tripleto encontramos [23, 24]

$$
h_{1}^{\mathrm{eff}}=J_{l} \mathbf{S}_{l} \cdot \mathbf{s}_{1}^{\prime}+J_{r} \mathbf{s}_{2}^{\prime} \cdot \mathbf{S}_{r} .
$$




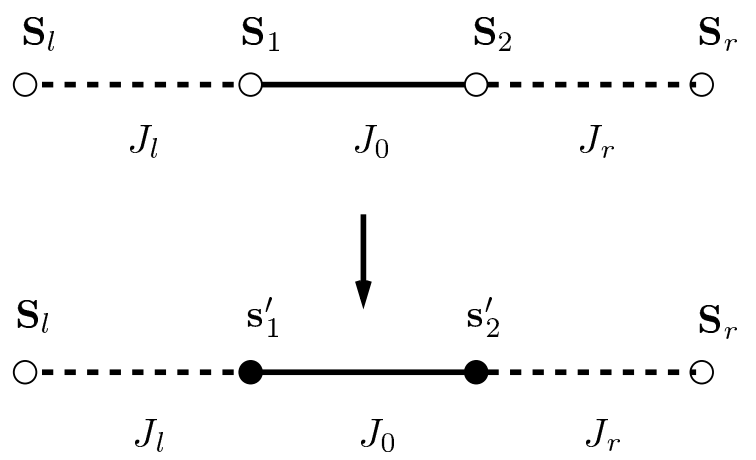

Figura 4.1: Procedimento de dizimação para um par de spins 1 de acordo com a segunda abordagem.

Essa regra reduz a escala de energia local de $\Delta=3 J_{0}$ (o gap entre os estados de singleto e o quintupleto de $h_{0}$ ) para $\Delta=J_{0}$ (o gap entre os estados de singleto e tripleto de $\left.h_{0}^{\text {eff }}\right)$. Mostramos no apêndice @ os resultados explícitos da teoria de perturbação em primeira ordem para todas as hamiltonianas envolvidas $h_{1}, h_{1}^{\text {eff }}$ e $h_{1}^{\text {exact }}$ que discutiremos a seguir.

Porém, a hamiltoniana efetiva da equação (4.7) não reproduz corretamente os elementos de matriz de $h_{1}$ entre os estados que conectam entre si os subespaços de singleto e tripleto. Para encontrarmos o resultado correto precisamos introduzir acoplamentos de segundos vizinhos, que dão origem ao resultado exato para a teoria de perturbação de primeira ordem para a hamiltoniana efetiva [22, 23, 24]

$$
h_{1}^{\text {exact }}=J_{l} \mathbf{S}_{l} \cdot\left(\alpha_{+} \mathbf{s}_{1}^{\prime}+\alpha_{-} \mathbf{s}_{2}^{\prime}\right)+J_{r}\left(\alpha_{-} \mathbf{s}_{1}^{\prime}+\alpha_{+} \mathbf{s}_{2}^{\prime}\right) \cdot \mathbf{S}_{r},
$$

com

$$
\alpha_{ \pm}=\frac{1 \pm \alpha}{2} \quad \text { e } \quad \alpha=\sqrt{\frac{8}{3}} \simeq 1.63
$$

Como as ligações de segundos vizinhos são ferromagnéticas, $\alpha_{-} J_{l, r} \simeq-0.316 J_{l, r}$, estas não introduzem frustração no sistema. Note também que embora as ligações de primeiros vizinhos, $\alpha_{+} J_{l, r} \simeq 1.32 J_{l, r}$, sejam maiores que as ligações originais, podemos verificar que os gaps $(\Delta)$ associados às ligações do bloco de quatro spins diminuíram quando comparados com $\Delta=3 J_{0}$ (veja a tabela 4.3 na seção 4.4), assim diminuindo de forma consistente a escala de energia.

A tabela 4.3 mostra todos os valores de escala de energia local, ou gaps $(\Delta)$ em função 
da configuração do bloco e do valor do parâmetro que conecta o bloco no caso mais geral de desordem aperiódica. Na segunda abordagem salientamos que em cada varredura para encontrar o maior parâmetro de energia local, usamos o parâmetro $\Delta$.

Devido ao fato de que as ligações ferromagnéticas não introduzem frustrações, Monthus et al. argumentaram que seria possível ignorá-las, sendo que ambas as equações (4.7) e (4.8) dão qualitativamente os mesmos resultados, enquanto quantitativamente dão o mesmo resultado para a maioria dos elementos de matriz da teoria de perturbação. A equação (4.7) forma então a base da segunda abordagem.

Como o termo perturbativo efetivo introduz objetos de spin $1 / 2$, é necessário considerar regras de renormalização que envolvem tanto spins $1 / 2$ quanto spins 1 para obter um esquema completo de renormalização.

Há a possibilidade de que o maior gap, ou parâmetro de energia local, seja um par composto por um spin $1 / 2\left(\mathbf{s}_{1}\right)$ e um spin $1\left(\mathbf{S}_{2}\right)$ conectados por um acoplamento $J_{0}$, interagindo com spins vizinhos $\mathbf{s}_{l}$ e $\mathbf{S}_{r}$ por meio dos acoplamentos $J_{l}$ e $J_{r}$, como ilustra a figura 4.2. O estado fundamental do par corresponde a um dubleto, dando origem a um spin $1 / 2$ efetivo $\mathbf{s}_{1}^{\prime}$. A teoria de perturbação em primeira ordem gera uma matriz efetiva dada por

$$
h^{\mathrm{eff}}=J_{l}^{\prime} \mathbf{s}_{l} \cdot \mathbf{s}_{1}^{\prime}+J_{r}^{\prime} \mathbf{s}_{1}^{\prime} \cdot \mathbf{S}_{r},
$$

com

$$
J_{l}^{\prime}=-\frac{1}{3} J_{l} \quad \text { e } \quad J_{r}^{\prime}=\frac{4}{3} J_{r}
$$

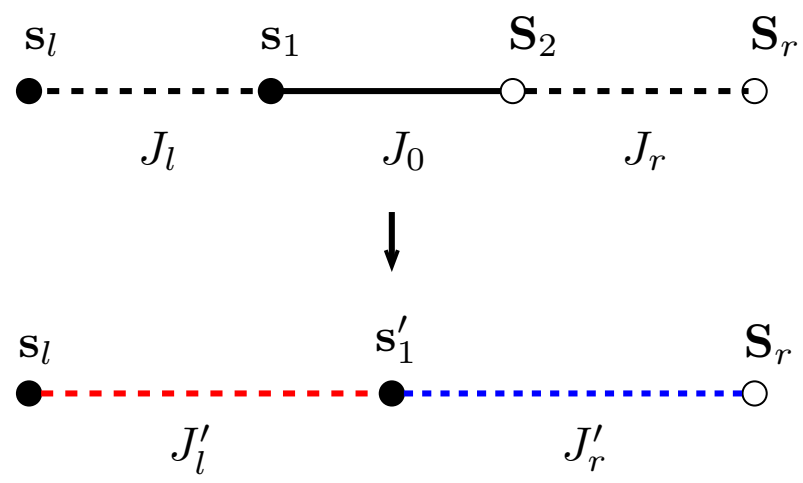

Figura 4.2: Procedimento de dizimação para uma par misto de spins de acordo com a segunda abordagem.

Note que em processos descritos pela última regra, são gerados acoplamentos ferro- 
magnéticos, que conectam somente pares de spin $1 / 2$. No caso em que a escala de energia é estabelecida por um tal acoplamento, digamos $-\left|J_{0}\right|$, que conecta $\mathbf{s}_{1}$ e $\mathbf{s}_{2}$, o estado fundamental é um tripleto, dando origem a um spin efetivo de módulo $1, \mathbf{S}_{1}^{\prime}$, como ilustra a figura 4.3. A teoria de perturbação em primeira ordem gera uma matriz efetiva dada por

$$
h^{\mathrm{eff}}=J_{l}^{\prime} \mathbf{S}_{l} \cdot \mathbf{S}_{1}^{\prime}+J_{r}^{\prime} \mathbf{S}_{1}^{\prime} \cdot \mathbf{S}_{r}, \quad \text { com } \quad J_{l, r}^{\prime}=\frac{1}{2} J_{l, r} .
$$
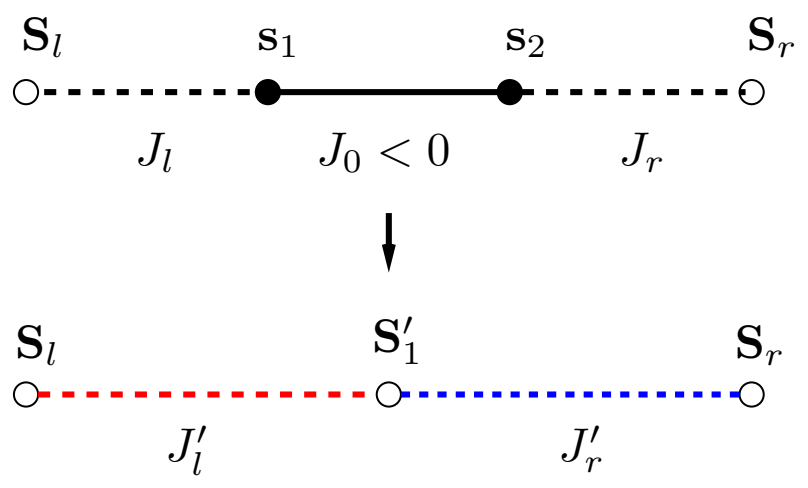

Figura 4.3: Procedimento de dizimação para um par de spins 1/2 acoplados ferromagneticamente de acordo com a segunda abordagem.

No dois casos anteriores não foi necessário discutir diferentes abordagens para dizimar o par de spins pois há apenas dois níveis de energia, portanto há apenas um modo de dizimar os estados excitados.

Para resumir a segunda abordagem sempre há 4 tipos de acoplamentos, e cada um deve ser dizimado de acordo com uma determinada regra. Utilizando a mesma notação do artigo [24], essas regras são:

- Regra 1: Um par de spins 1/2 conectados por um acoplamento ferromagnético [figura 4.3, equação (4.12)];

- Regra 2: Um par de spins 1/2 conectados por um acoplamento antiferromagnético [figura 3.1, equações (3.4) e (3.5)];

- Regra 3: Um par misto de spins acoplados antiferromagneticamente [figura 4.2. equações (4.10) e (4.11)];

- Regra 4: Um par de spins 1 conectados por um acoplamento antiferromagnético [figura 4.1, equação (4.7)]. 
Para decidirmos qual regra devemos aplicar e em qual sequência, ou seja, qual acoplamento define a escala de energia global, devemos olhar para o gap local $\Delta$ entre o estado fundamental e o primeiro estado excitado que descartamos no processo de renormalização. Para as quatro diferentes regras temos

$$
\begin{aligned}
\Delta_{1} & =-J_{0}=\left|J_{0}\right|, \\
\Delta_{2} & =J_{0}, \\
\Delta_{3} & =\frac{3}{2} J_{0}, \\
\Delta_{4} & =3 J_{0} .
\end{aligned}
$$

O esquema do SDRG para a cadeia de spin 1 corresponde à aplicação iterativa das regras de renormalização, seguindo sempre pela ordem do maior parâmetro $\Delta$, e escolhendo a regra apropriada a esse parâmetro.

\subsection{Terceira abordagem.}

A terceira abordagem consiste em usar a hamiltoniana exata de primeira ordem em teoria de perturbação $h_{1}^{\text {exact }}$, equação (4.8), como hamiltoniana efetiva local proveniente da renormalização de uma par de spins 1. Entre as regras de renormalização precisamos modificar a quarta de acordo com a equação 4.8. Com a introdução das ligações de segundos vizinhos, ou mesmo mais longas, é possível que os spins $\mathbf{S}_{1}$ e $\mathbf{S}_{2}$ esteja fortemente acoplados, enquanto ambos estejam fracamente acoplados com vários spins vizinhos, ver figura 4.4. Portanto a hamiltoniana exata de primeira ordem deve ser escrita como

$$
h_{1}^{\text {exact }}=\sum_{i=1}^{n_{l}} J_{l}^{(i)} \mathbf{S}_{l}^{(i)} \cdot\left(\alpha_{+} \mathbf{s}_{1}^{\prime}+\alpha_{-} \mathbf{s}_{2}^{\prime}\right)+\sum_{i=1}^{n_{r}} J_{r}^{(i)}\left(\alpha_{-} \mathbf{s}_{1}^{\prime}+\alpha_{+} \mathbf{s}_{2}^{\prime}\right) \cdot \mathbf{S}_{r}^{(i)}
$$

onde $n_{l}$ o número de spins $\mathbf{S}_{l}^{(i)}$ que se acoplam fracamente a $\mathbf{S}_{1}$ via $J_{l}^{(i)}$, e $n_{r}$ é o número de spins $\mathbf{S}_{r}^{(i)}$ que se acoplam fracamente a $\mathbf{S}_{2}$ via $J_{r}^{(i)}$. Assim, a regra 4 torna-se agora

- Regra 4': Um par de spins 1 acoplados por uma ligação antiferromagnética [figura 4.4. equação (4.14)].

Note que na figura 4.4 ilustramos o caso $n_{l}=n_{r}=1$. 


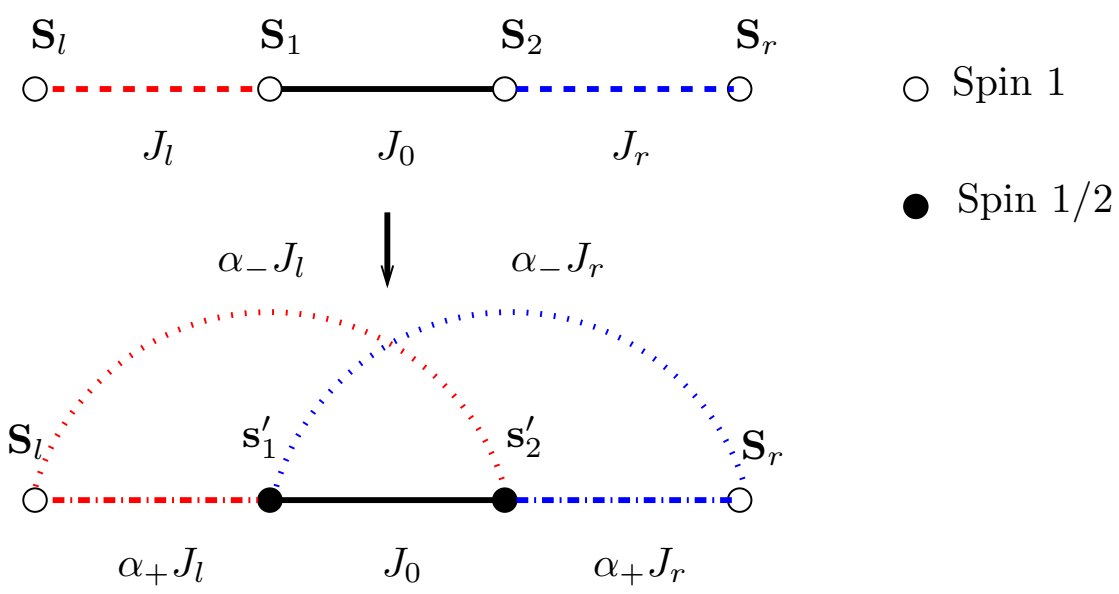

Figura 4.4: Procedimento de dizimação para um par de spins 1 de acordo com a terceira abordagem.

\subsection{Adaptação do método MDH no caso aperiódico.}

No caso de modelos desordenados onde a desordem é aperiodica, gerada por regras de substituição, sempre há a possibilidade de encontrar blocos compostos por mais de uma ligação, como no caso da sequência de Fibonacci com $J_{a}>J_{b}$. Para lidar com esses casos precisamos expandir e generalizar as regras de dizimação, como feito para a cadeia aperiódica de spins 1/2 [33, 34]. O ponto de partida é encontrar o bloco de spins que tem o maior gap local, e renormalizá-lo para uma ligação efetiva entre os vizinhos do bloco, ou para um spin ou dois spins efetivos, de acordo com os níveis de energia mais baixos da matriz original que se deseja preservar. Os acoplamentos efetivos são então calculados por teoria de perturbação de primeira ou segunda ordem. Implementações numéricas são convenientes tanto para o cálculo dos acoplamentos efetivos via teoria de perturbação, quanto para aplicar o grupo de renormalização a cadeias quânticas aperiódicas com modulação fraca, onde a dizimação desses blocos se torna essencial.

Mostraremos a seguir um exemplo de como formular uma regra de dizimação para blocos formados apenas por spins 1, conectados antiferromagneticamente pelo parâmetro que induz ao maior gap local. Escolhemos a segunda abordagem, pois essa também contém variáveis de spin $1 / 2$, sendo mais simples que a terceira abordagem por não conter ligações além de primeiros vizinhos. Para a primeira abordagem temos um caso mais simples com uma formulação muito semelhante à formulação da cadeia de spin $1 / 2$, que pode ser 
encontrada na referência [34].

Novamente separamos a contribuição do bloco com o maior gap local no hamiltoniano, que agora contém $n$ spins conectados por $J_{0}$, como

$$
\begin{aligned}
h & =h_{0}+h_{1}, \\
h_{0} & =J_{0} \sum_{i=1}^{n-1} \mathbf{S}_{i} \cdot \mathbf{S}_{i+1}, \\
h_{1} & =J_{l} \mathbf{S}_{l} \cdot \mathbf{S}_{1}+J_{r} \mathbf{S}_{n} \cdot \mathbf{S}_{r} .
\end{aligned}
$$

Há dois casos diferentes a serem considerados. O primeiro ocorre quando o número de spins do bloco central é ímpar, caso em que, como os acoplamentos são antiferromagnéticos, o espectro do bloco tem um estado fundamental triplamente degenerado e um primeiro estado excitado não-degenerado. Estes níveis são compatíveis com aqueles produzidos por um par de spins $1 / 2$ acoplados ferromagneticamente $\left(J_{0}^{\prime}<0\right)$.

Podemos criar uma imagem pictórica para melhor entender o estado fundamental desse bloco usando o conceito, ou representação, de ligações de valência [7, 19]. Nessa representação, consideramos cada spin 1 sendo formado por um par de spins $1 / 2$ indistinguíveis e simetrizados, localizados no mesmo sítio. Um dos spins $1 / 2$ desse sítio forma um estado de singleto com outro spin $1 / 2$ do sítio vizinho à direita, enquanto o segundo spin $1 / 2$ forma outro estado de singleto com um spin $1 / 2$ do sítio vizinho à esquerda.

Essa situação está ilustrada na figura 4.5(b) para um bloco de tamanho $n=3$. Assim, em cada ligação há também um estado de singleto de spins $1 / 2$, restando nos extremos dos blocos spins 1/2 efetivos desemparelhados. Esses spins desacoplados serão os spins efetivos que usaremos como aproximação para os níveis de menor energia do bloco. Notase que esses spins estão paralelos entre si, e, portanto devem ser conectados por uma ligação efetiva ferromagnética.

O outro caso ocorre quando o número de spins do bloco é par, situação em que o espectro da matriz original tem um estado fundamental não degenerado e um primeiro estado excitado triplamente degenerado. Esses níveis são compatíveis com os níveis produzidos por um par de spins $1 / 2$ acoplados por uma ligação antiferromagnética. A figura 4.6 ilustra essa situação, bem como a representação pictórica em termos de ligações de valência.

Desse modo o hamiltoniano efetivo sempre será formado por um par de spins $1 / 2$. 
(a)

$\mathbf{S}_{l}$

$J_{l}$

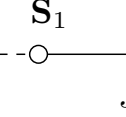

(b)
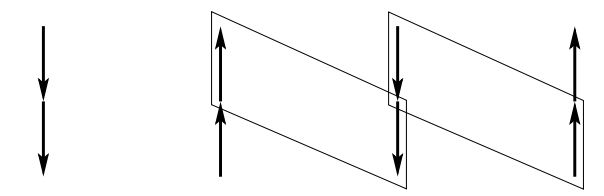

(c)

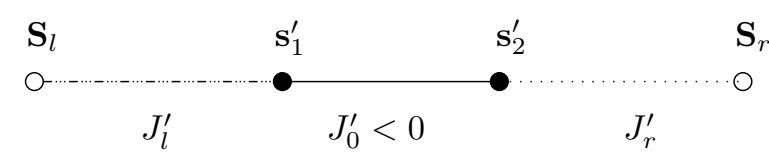

$\mathbf{S}_{r}$

○ Spin 1

- Spin $1 / 2$

Figura 4.5: (a) Bloco com três spins fortemente acoplados. (b) Representação de ligações de valência. (c) Spins e parâmetros efetivos.
(a)<smiles>C1C[SiH2]C[SiH2]1</smiles>
$\mathbf{S}_{1}$
$\mathbf{S}_{2}$
$\mathbf{S}_{3}$
$\mathbf{S}_{4}$
$\mathbf{S}_{r}$
$J_{l} \quad J_{0}$

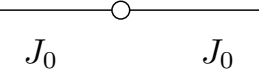
$J_{r}$
○ Spin 1
(b)
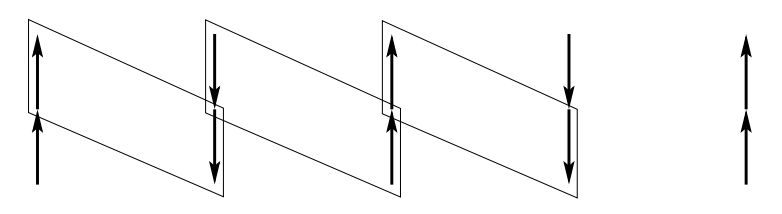
- Spin $1 / 2$
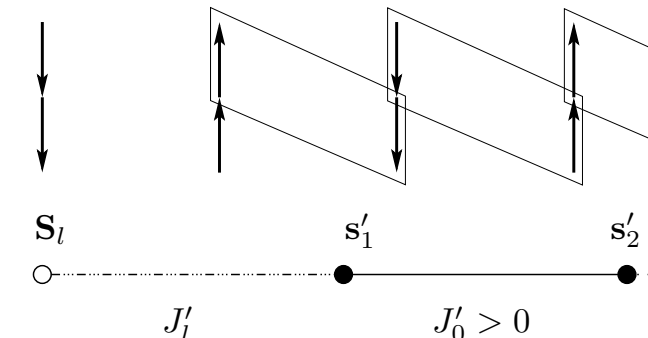
$\mathrm{s}_{2}^{\prime}$
$\mathbf{S}_{r}$
(c)

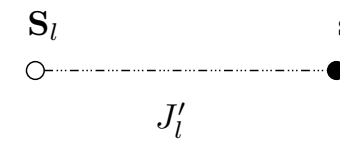
$J_{0}^{\prime}>0$
$J_{r}^{\prime}$

Figura 4.6: (a) Bloco com quatro spins fortemente acoplados. (b) Representação de ligações de valência. (c) Spins e parâmetros efetivos.

Resta agora saber como calcular os parâmetros efetivos. A contribuição no hamiltoniano efetivo desses dois casos é dada por

$$
\begin{aligned}
h^{\prime} & =h_{0}^{\prime}+h_{1}^{\prime}, \\
h_{0}^{\prime} & =J_{0}^{\prime} \mathbf{s}_{1}^{\prime} \cdot \mathbf{s}_{2}^{\prime}, \\
h_{1}^{\prime} & =J_{l}^{\prime} \mathbf{S}_{l} \cdot \mathbf{s}_{1}^{\prime}+J_{r}^{\prime} \mathbf{s}_{2}^{\prime} \cdot \mathbf{S}_{r} .
\end{aligned}
$$

Considerando $h_{1}^{\prime}$ como um perturbação sobre os estados de $h_{0}^{\prime}$ e impondo que os elementos de matriz da teoria de perturbação sejam iguais aos elementos de matriz produzidos pela teoria de perturbação envolvendo as equações da hamiltoniana original (4.15), projetados nos dois estados de menor energia, obteremos o fator de proporcionalidade $\gamma$ 
entre os parâmetros originais e os parâmetros efetivos, $J_{i}=\gamma J_{i}^{\prime}$, da matriz perturbativa $h_{1}^{\prime}$. Para tanto, temos que avaliar as expressões

$$
\begin{gathered}
\left\langle\Psi_{1}^{\alpha}\left|\mathbf{S}_{1}\right| \Psi_{1}^{\alpha}\right\rangle, \\
\left\langle\Psi_{1}^{\prime \alpha}\left|\mathbf{S}_{1}^{\prime}\right| \Psi_{1}^{\prime \alpha}\right\rangle,
\end{gathered}
$$

onde $\alpha=+1,0,-1$ representa os primeiros estados excitados da hamiltoniana original. Esses são os elementos de matriz que surgem na teoria de perturbação em primeira ordem.

Repare que os spins das vizinhanças não fazem diferença nesse cálculo, pois são os mesmos spins em ambos os hamiltonianos, ver apêndice A. Devido à simetria do modelo, podemos executar esse cálculo apenas para a componente z. A segunda expressão de (4.17) pode ser calculada analiticamente, resultando em

$$
\left\langle\Psi_{1}^{\prime+}\left|s_{1}^{\prime z}\right| \Psi_{1}^{\prime+}\right\rangle=\frac{1}{2}
$$

onde escolhermos particurlamente $\alpha=+1$.

A primeira expressão de (4.17) pode ser calculada numericamente para obter o fator $\gamma$, que é a razão entre os dois elementos de matriz calculados.

O cálculo do parâmetro $J_{0}^{\prime}=\Gamma J_{0}$, da matriz composta pelo par central de spins, é feito exigindo-se que o gap do par de spins $1 / 2$ seja igual ao primeiro gap do bloco original, ou seja, $J_{0}^{\prime}=\epsilon(1)-\epsilon(0)$, onde $\epsilon(i)$ representa o i-ésimo nível de energia do bloco original.

Podemos empregar esse método para calcular os parâmetros efetivos de blocos de vários tamanhos. Os resultados são mostrados na tabela 4.1. Repare que para $n=2$ os resultados coincidem com os resultados analíticos.

\begin{tabular}{rrrr}
\hline \hline $\mathrm{n}$ & $\gamma$ & $\Gamma$ & $\Delta / J_{0}$ \\
\hline 2 & 1.0000 & 1.0000 & 3.0000 \\
3 & 1.5000 & -1.0000 & 2.0000 \\
4 & 1.0106 & 0.5092 & 1.8545 \\
5 & 1.3192 & -0.5466 & 1.4249 \\
6 & 1.0112 & 0.3078 & 1.3517 \\
\hline
\end{tabular}

Tabela 4.1: Parâmetros calculados numericamente para a regra 4 de renormalização para blocos com $n$ spins.

Repare que o gap se torna menor conforme o bloco aumenta. Esperamos que para 


\section{Grupo de renormalização de desordem forte para a cadeia de}

blocos muito grandes, o valor de $\Delta$ se aproxime das estimativas para o gap de Haldane, que é dado por $\sim 0,41 J_{0}$ [4].

Nas tabelas seguintes, listamos os gaps locais correspondentes a vários blocos produzidos pelas sequências aperiódicas usadas nesse trabalho, onde usamos algumas variações do método descrito há pouco. A tabela 4.2 lista os blocos relevantes para a primeira abordagem, enquanto a tabela 4.3 mostra os blocos relevantes para a segunda e terceira abordagens. A última coluna de cada tabela mostra os blocos renormalizados, onde uma linha reta corresponde a um acoplamento efetivo entre os spins vizinhos do bloco. Acoplamentos efetivos adicionais podem ocorrer, veja as figuras de 3.1 à 4.4 .

\begin{tabular}{cccc}
\hline \hline n (tamanho do bloco) & configuração & $\Delta / J$ (gap) & bloco renormalizado \\
\hline 2 & $\circ-\circ$ & 1.0 & - \\
3 & $\circ-0-\circ$ & 1.0 & $\circ$ \\
4 & $\circ-\circ-\circ-\circ$ & 0.5092 & - \\
\hline notação: & $\circ=$ spin 1 & & \\
\hline
\end{tabular}

Tabela 4.2: Gaps locais $\Delta$, em unidades da ligação $J$ que conecta os spins em cada um dos vários blocos relevantes para a primeira abordagem. A última coluna mostra o correspondente bloco renormalizado.

\begin{tabular}{|c|c|c|c|}
\hline n (tamanho do bloco) & configuração & $\Delta /|J|($ gap $)$ & bloco renormalizado \\
\hline 2 & $0-0$ & 3.0 & $\bullet \bullet \bullet\left(J^{\prime}>0\right)$ \\
\hline 2 & $\bullet-0$ & 1.5 & $\bullet$ \\
\hline 2 & $\bullet \bullet(J>0)$ & 1.0 & $-\left(J^{\prime}>0\right)$ \\
\hline 2 & $\bullet \bullet(J<0)$ & 1.0 & ० \\
\hline 3 & $0-0-0$ & 2.0 & $\bullet \longrightarrow\left(J^{\prime}<0\right)$ \\
\hline 3 & $\bullet-0-\bullet$ & 1.0 & $-\left(J^{\prime}>0\right)$ \\
\hline 3 & $0-0-\bullet$ & 1.5 & $\bullet$ \\
\hline 3 & $\bullet-\bullet-\bullet$ & 1.0 & $\bullet$ \\
\hline 4 & $0-0-0-0$ & 1.8545 & $\bullet \bullet\left(J^{\prime}>0\right)$ \\
\hline 4 & $\bullet-0-0-\bullet$ & 1.9142 & $\bullet-\left(J^{\prime}>0\right)$ \\
\hline 4 & $0-0-0-\bullet$ & 1.0778 & $\bullet$ \\
\hline notação: & $\sin 1 ; \bullet=$ spil & & \\
\hline
\end{tabular}

Tabela 4.3: Gaps locais $\Delta$, em unidades da ligação $J$ que conecta os spins em cada um dos vários blocos relevantes para a segunda abordagem. A última coluna mostra o correspondente bloco renormalizado, com spins conectados por $J^{\prime}$. 


\section{Capítulo 5}

\section{Cadeia de Fibonacci-Heisenberg de spin 1.}

Neste capítulo aplicamos as três diferentes abordagens do SDRG para estudar os efeitos das flutuações geométricas geradas pela sequência de Fibonacci na cadeia de Heisenberg de spin 1. A sequência de Fibonacci é produzida pela iteração da regra de substituição

$$
\sigma_{\mathrm{fb}}:\left\{\begin{array}{l}
a \rightarrow a b \\
b \rightarrow a
\end{array} .\right.
$$

Começando com uma única letra ( $a$ ou $b$ ), geramos uma sequência abaababaabaab $\cdots$, que não tem período.

A cadeia de Heisenberg de spin $1 / 2$ com acoplamentos $J_{i} \in\left\{J_{a}, J_{b}\right\}$ seguindo a sequência de Fibonacci permanece crítica (sem gap entre o estado fundamental e os estados excitados) para qualquer valor finito da razão $J_{b} / J_{a}$. Como a dimerização forçada gera um gap na cadeia, as flutuações geométricas das sequências aperiódicas são aquelas medidas para subsequências de duas letras. Como estudado por Hermisson [42], essas flutuações crescem com o comprimento como uma lei de potências, com expoente $\omega$ relacionado a regra de substituição de pares de letras, ao invés de somente letras.

No caso da sequência de Fibonacci, esse expoente é $\omega=0$, que está em contraste com a cadeia de spins aleatória, para a qual $\omega=\frac{1}{2}$. Assim, as flutuações geométricas associadas à sequência de Fibonacci são muito mais fracas do que aquelas produzidas por uma distribuição aleatória de acoplamentos. Mesmo assim a sequência de Fibonacci induz 
dramáticas mudanças no comportamento de baixas temperaturas da cadeia de Heisenberg de spin 1/2 [33, 34]. Como mostraremos a seguir esse não é o caso para a cadeia de spin 1 .

Nas seções a seguir apresentamos os resultados da aplicação das três diferentes abordagens do SDRG definidas na seção anterior para a cadeia de Fibonacci-Heisenberg de spins 1. Também discutimos as diferenças entre a segunda e as demais abordagens. Note que a segunda abordagem leva a conclusões qualitativamente erradas para o comportamento de baixas temperaturas.

\subsection{SDRG: Primeira abordagem.}

Na figura 5.1(a) mostramos alguns spins da extremidade esquerda de uma cadeia de Fibonacci-Heisenberg. Sempre assumimos a cadeia inicial com $J_{b}>J_{a}$, além disso também assumimos a condição de modulação forte $J_{b} \gg J_{a}$, para aplicarmos a primeira abordagem evitando problemas com a auto-consistência no início do procedimento de renormalização.

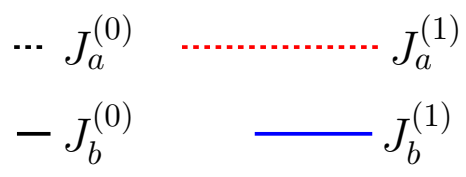

\section{9}

(a)

(b)

Figura 5.1: Renormalização de uma cadeia de Fibonacci-Heisenberg de spin 1 de acordo com a primeira abordagem do SDRG. (a) Distribuição de acoplamentos da cadeia de Fibonacci-Heisenberg de spin 1. (b) Cadeia efetiva obtida a partir da primeira abordagem do SDRG após uma varredura ao longo da cadeia.

De acordo com o procedimento usual da primeira abordagem todas as ligações $J_{b}$, que aparecem destacados na figura 5.1(a), são dizimadas na primeira varredura, dando origem a acoplamentos efetivos. Entre os spins 1 e 4 na figura 5.1(a) há apenas um par conectado por $J_{b}$, e sua dizimação resulta em uma ligação efetiva $J_{b}^{\prime}$, pela aplicação da equação (4.4). Porém há outra ligação efetiva, $J_{a}^{\prime}$, que aparece por exemplo entre os spins 
4 e 9 pela dizimação em sequência de ligações $J_{b}$ conectando os spins 5-6 e 7-8. Assim temos

$$
J_{a}^{\prime}=\left(\frac{4}{3}\right)^{2} \frac{J_{a}^{3}}{J_{b}^{2}} \quad \text { e } \quad J_{b}^{\prime}=\frac{4}{3} \frac{J_{a}^{2}}{J_{b}} .
$$

Note que, ao exigirmos que a ligação efetiva $J_{b}^{\prime}$ seja menor do que a ligação original dizimada $J_{b}$, de modo que a dizimação gere uma redução da escala de energia, devemos ter $J_{b}>\sqrt{4 / 3} J_{a}$, que é justamente a condição de auto-consistência da primeira abordagem.

Como indicado pela figura 5.1 (b), a dizimação de todas a ligações $J_{b}$ gera uma modulação de Fibonacci nas ligações efetivas (desconsiderando a primeira ligação que é apenas um efeito de borda que pode ser desprezado). Fica claro que cada nova varredura do SDRG gera novamente uma sequência de Fibonacci, e assim por diante. Portanto podemos definir equações recursivas para os parâmetros efetivos, assim como para a razão efetiva entre os parâmetros, que são dadas por

$$
\begin{aligned}
J_{a}^{(j+1)} & =\left(\frac{4}{3}\right)^{2} \frac{\left[J_{a}^{(j)}\right]^{3}}{\left[J_{b}^{(j)}\right]^{2}}, \quad J_{b}^{(j+1)}=\frac{4}{3} \frac{\left[J_{a}^{(j)}\right]^{2}}{J_{b}^{(j)}}, \\
r^{(j+1)} & \equiv \frac{J_{b}^{(j+1)}}{J_{a}^{(j+1)}}=\left(\frac{3}{4}\right) r^{(j)},
\end{aligned}
$$

onde $j$ designa o passo do SDRG, $j=0$ correspondendo aos parâmetros da cadeia original.

Note que a razão entre os parâmetros efetivos decresce com a iteração do SDRG. Isto significa que a cadeia efetiva se torna cada vez mais próxima de uma cadeia uniforme quando a escala de energia é reduzida. Concluímos assim que a modulação de Fibonacci não induz o sistema a uma transição para uma fase de singleto aperiódico mesmo que a razão inicial seja arbitrariamente grande, diferentemente do que ocorre com a cadeia de Fibonacci-Heisenberg de spin 1/2 [33, 34].

Esperamos que a cadeia permaneça na fase de Haldane, porém com um gap menor que depende da razão inicial $r=J_{b} / J_{a}$. Uma estimativa desse gap pode ser fornecida pelo valor do acoplamento efetivo na escala de energia para a qual a razão efetiva se torna de 
ordem 1. Isso acontece após $j^{*}$ iterações do esquema de SDRG, com

$$
j^{*}=\left[\frac{\ln r}{\ln 4 / 3}\right]_{\text {inteiro }} .
$$

Com as equações de recorrência logo acima pode-se encontrar o valor do acoplamento efetivo $J_{b}$ como função da iteração $j$ e dos parâmetros iniciais,

$$
J_{b}^{(j)}=r_{0}^{-2(j)} \gamma^{(j)^{2}} J_{b}^{(0)}
$$

a partir da qual podemos estimar o gap $\Delta(r) \sim J_{b}^{\left(j^{*}\right)}$. Usando a expressão para a iteração final $j^{*}$, podemos concluir que o gap se comporta como

$$
\Delta(r) \sim r^{-\frac{\ln r}{\ln (4 / 3)}} J_{b}
$$

a menos de uma constante multiplicativa. Aplicando o logaritmo em ambos os lados, esse último resultado pode ser reescrito como

$$
\ln \Delta(r) \sim \ln J_{b}-\frac{\ln ^{2} r}{\ln (4 / 3)}
$$

evidenciando que o gap decresce assintoticamente conforme aumenta-se a razão inicial.

Também é possível determinar como cresce o comprimento das ligações conforme iteramos o SDRG. Se denotamos por $\ell_{a}^{(j)}$ e $\ell_{b}^{(j)}$ os comprimentos das ligações fracas e fortes após $j$ iterações do SDRG, pela figura 5.1percebemos que a relação entre os comprimentos efetivos pode ser escrita em uma forma matricial,

$$
\left[\begin{array}{l}
\ell_{a}^{(j+1)} \\
\ell_{b}^{(j+1)}
\end{array}\right]=\left[\begin{array}{ll}
3 & 2 \\
2 & 1
\end{array}\right] \cdot\left[\begin{array}{c}
\ell_{a}^{(j)} \\
\ell_{b}^{(j)}
\end{array}\right],
$$

então assintoticamente os comprimentos crescem como

$$
\ell_{a}^{(j)} \sim \ell_{b}^{(j)} \sim \tau^{j}
$$

com $\tau=2+\sqrt{5}$ sendo o maior autovalor da matriz acima, correspondendo ao fator de escala do grupo de renormalização. Assumindo o comprimento inicial $\ell_{a}^{(0)}=\ell_{b}^{(0)}=1$, o 
comprimento assintótico das ligações mais fortes é dado por

$$
\ell_{b}^{(j)} \simeq \frac{1+\sqrt{5}}{2 \sqrt{5}} \tau^{j} \equiv c \tau^{j}
$$

Uma estimativa para o comprimento de correlação para a cadeia de Fibonacci-Heisenberg de spin 1 é dada pelo comprimento da ligação mais forte em dada iteração do SDRG quando a razão efetiva se torna da ordem 1. Assim temos

$$
\xi \sim \ell_{b}^{\left(j^{*}\right)} \simeq c r^{\nu}
$$

mostrando que o comprimento de correlação diverge no limite de modulação infinita como uma lei de potência com um expoente de valor muito alto dado por

$$
\nu=\frac{\ln \tau}{\ln \frac{4}{3}} \simeq 5.02
$$

\subsection{SDRG: Segunda abordagem.}

Agora estudaremos as conclusões que podem ser extraídas da segunda abordagem considerando novamente o caso de modulação forte $J_{b} \gg J_{a}$.

A figura 5.2 ilustra os passos necessários para obter os parâmetros efetivos na cadeia de Fibonacci-Heisenberg de acordo com a segunda abordagem. A cadeia original é mostrada na figura 5.2(a). Aplicando a regra 4 da seção 4.2 em todas as ligações $J_{b}$ que conectam pares de spin 1, esses são substituídos por pares de spin 1/2, como ilustra a figura 5.2 (b). Como assumimos $J_{b} \gg J_{a}$, o próximo passo dizima todos os pares de spin $1 / 2$ conectados por $J_{b}$, dando origem aos acoplamentos efetivos

$$
J_{a}^{\prime}=\left(\frac{1}{2}\right)^{2} \frac{J_{a}^{3}}{J_{b}^{2}} \quad \text { e } \quad J_{b}^{\prime}=\frac{1}{2} \frac{J_{a}^{2}}{J_{b}} .
$$

Novamente, ignorando o efeito na borda esquerda na figura 5.2(c), os acoplamentos efetivos são modulados pela sequência de Fibonacci.

Pelas equações acima, é fácil ver que os valores dos acoplamentos efetivos preditos pela segunda abordagem são significantemente menores do que aqueles preditos pela primeira abordagem. Isto induz diferenças quando usamos os acoplamentos efetivos $J_{b}$ como es- 


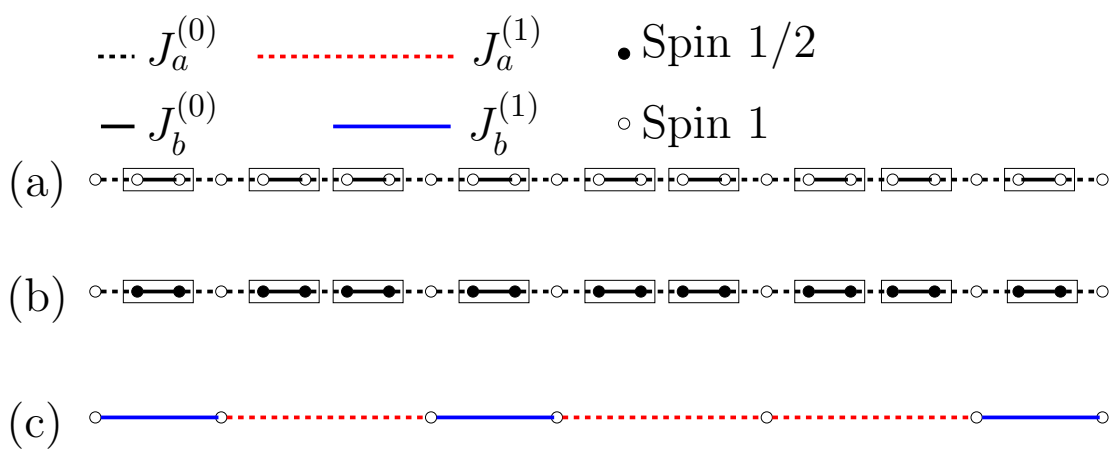

Figura 5.2: Renormalização de uma cadeia de Fibonacci-Heisenberg de spin 1 de acordo com a segunda abordagem do SDRG. (a) A cadeia original. (b) Pares de spin 1 conectados pela ligação forte $J_{b}$ são substituídos por pares de spin $\frac{1}{2}$. (c) Pares de spin $\frac{1}{2}$ são dizimados, dando origem aos acoplamentos efetivos entre os spins 1 restantes.

timativa para os níveis de energia da cadeia de Fibonacci-Heisenberg. Também é fácil ver que, ao contrário do ocorrido na primeira abordagem, a razão entre os acoplamentos efetivos predita pela segunda abordagem,

$$
r^{\prime}=\frac{J_{b}^{\prime}}{J_{a}^{\prime}}=2 \frac{J_{b}}{J_{a}}=2 r
$$

é maior do que a razão inicial $r$. Portanto, de acordo com a segunda abordagem, a razão efetiva cresce iterativamente, quando a escala de energia diminui. Assim podemos afirmar que a modulação de Fibonacci induziria uma transição para uma fase de singleto aperiódico sem gap, análoga à observada na cadeia de Fibonacci-Heisenberg de spin 1/2. Está conclusão é qualitativamente incorreta, pois, como veremos adiante, levando em conta as ligações de segundos vizinhos que foram negligenciadas na segunda abordagem recuperamos as predições da primeira abordagem.

\subsection{SDRG: Terceira abordagem.}

Quando aplicamos a terceira abordagem na cadeia de Fibonacci-Heisenberg seguindo o esquema das seções 4.2 e 4.3, após substituir todos os pares de spin 1 conectados por $J_{b}$ por pares de spin $1 / 2$, surgem ligações de segundos e terceiros vizinhos como ilustra a figura 5.3(b). Repare que o acoplamento entre os spins 5 e 8 na figura 5.3(b) surge devido a aplicação sequencial da regra $4^{\prime}$ nos pares separados por apenas uma ligação. 


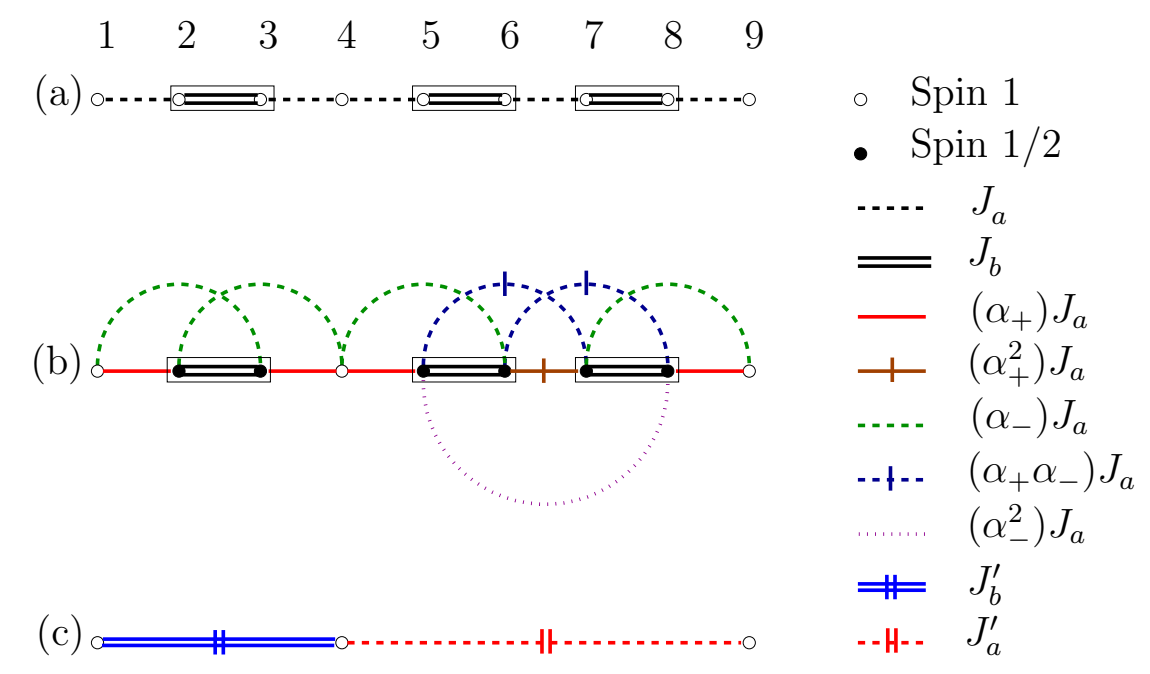

Figura 5.3: Renormalização da cadeia de Fibonacci-Heisenberg de spin 1 de acordo com a terceira abordagem. (a) A cadeia original. (b) Pares de spin 1 conectados por $J_{b}$ são substituídos por pares de spin $1 / 2$, e ligações de segundos e terceiros vizinhos são criadas. (c) Pares de spin $1 / 2$ são dizimados, dando origem à acoplamentos efetivos entre os spins 1 remanescentes.

Para $J_{b}>\alpha_{+}^{2} J_{a} \simeq 1.73 J_{a}$, o maior gap local na figura 5.3(b) é dado novamente pela ligação entre primeiros vizinhos $J_{b}$ (veja a seção 4.4), os quais devem ser dizimados dando origem aos acoplamentos efetivos ilustrados na figura 5.3(c). Este procedimento é diferente para as ligações $J_{b}$ que são separadas de outras ligações $J_{b}$ por pelo menos duas ligações fracas $J_{a}$ (como a ligação entre os spins 2 e 3 na figura) e para os pares conectados por $J_{b}$ separados por apenas uma ligação $J_{a}$ (como na sequência de ligações entre os spins 5 e 8 ).

No primeiro caso temos que tratar todas as ligações fracas (primeiros e segundos vizinhos) como perturbação sobre a hamiltoniana

$$
h_{0}=J_{b} \mathbf{s}_{2} \cdot \mathbf{s}_{3},
$$

de acordo com a teoria de perturbação de segunda ordem análoga àquela da equação (3.4), para maiores detalhes veja o apêndice B. O resultado é um acoplamento efetivo entre os spins 1 e 4 na figura 5.3, dado por

$$
J_{b}^{\prime}=\frac{\left(\alpha_{+}-\alpha_{-}\right)^{2}}{2} \frac{J_{a}^{2}}{J_{b}}=\frac{4}{3} \frac{J_{a}^{2}}{J_{b}} .
$$


Já no segundo caso, para evitar ambiguidades que surgem na escolha da ordem na qual as ligações $J_{b}$ devem ser dizimadas, e que causam um aumento da escala de energia (inconsistência do SDRG), devemos utilizar teoria de perturbação em terceira ordem na qual todas as ligações fracas (de primeiros e segundos vizinhos) são tratadas como uma perturbação sobre a hamiltoniana que contém dois pares conectados por $J_{b}$,

$$
h_{0}=J_{b} \mathbf{s}_{5} \cdot \mathbf{s}_{6}+J_{b} \mathbf{s}_{7} \cdot \mathbf{s}_{8} .
$$

Como detalhamos no apêndice B, esse procedimento dá origem a uma ligação efetiva entre os spins 4 e 9, dada por

$$
J_{a}^{\prime}=\frac{\left(\alpha_{+}-\alpha_{-}\right)^{4}}{4} \frac{J_{a}^{3}}{J_{b}^{2}}=\left(\frac{4}{3}\right)^{2} \frac{J_{a}^{3}}{J_{b}^{2}} .
$$

Assim, comparando os resultados acima com a equação (5.2), vemos que para $J_{b}>$ $\alpha_{+}^{2} J_{a}$ a terceira abordagem gera exatamente o mesmo resultado da primeira abordagem. Portanto, levando em conta corretamente as ligações mais longas geradas pelo SDRG consertamos as predições qualitativamente incorretas da segunda abordagem que implicava que a modulação forte de Fibonacci induz uma fase sem gap, dominada pela aperiodicidade, na cadeia de Heisenberg de spin 1.

Concluímos por meio do SDRG que as flutuações geométricas da sequência de Fibonacci não destroem a fase de Haldane, ou o gap de Haldane. 


\section{Capítulo 6}

\section{Cadeia de Heisenberg-6-3 de spin 1.}

Estudamos agora os efeitos das flutuações geométricas induzidas pelos acoplamentos seguindo a sequência 6-3 na cadeia de Heisenberg de spin 1. A sequência 6-3 é definida pela regra

$$
\sigma_{6-3}:\left\{\begin{array}{l}
a \rightarrow b a b a a a \\
b \rightarrow b a a
\end{array} .\right.
$$

Começando por uma letra $a$ ou $b$, geramos uma sequência do tipo baababaaababaaa $\cdots$. O expoente de flutuação geométrica que caracteriza a flutuação para pares de letras na sequência 6-3 segundo Hermisson [42] é $\omega=\ln 2 / \ln 5 \simeq 0.43$. Assim esperamos tanto para a cadeia de spin 1/2 quanto, no limite de modulação forte, para a cadeia de spin 1 um comportamento de escala dinâmico exponencial

$$
\Delta(\ell) \sim \exp \left(\ell / \ell_{0}\right)^{\omega}
$$

com $l_{0}$ sendo uma constante não universal. Como descrevemos a seguir, este é exatamente o que obtemos pelo SDRG, quando a desordem é suficientemente forte.

\subsection{SDRG: Primeira abordagem.}

A figura 6.1(a) ilustra a distribuição de acoplamentos da sequência 6-3. Assumimos novamente $J_{b}>J_{a}$, e a primeira varredura do SDRG gera dois acoplamentos efetivos, do mesmo modo como ocorreu com a cadeia de Fibonacci-Heisenberg (veja a figura 5.1). Além desses, há um acoplamento remanescente $J_{a}$ que pode ser interpretado como um 
terceiro tipo de acoplamento efetivo, então podemos escrever

$$
J_{1}^{(0)}=J_{a}, \quad J_{2}^{(0)}=\left(\frac{4}{3}\right) \frac{J_{a}^{2}}{J_{b}}, \quad J_{3}^{(0)}=\left(\frac{4}{3}\right)^{2} \frac{J_{a}^{3}}{J_{b}^{2}},
$$

desde de que $J_{b}>\sqrt{4 / 3} J_{a}$.

$-J_{b} \cdots J_{a}$

(a)

(b)

$$
J_{3}^{(0)} \quad J_{1}^{(0)} \quad J_{2}^{(0)}
$$

Figura 6.1: (a) Distribuição de acoplamentos da cadeia de spin 1 de acordo com a sequência 6-3. (b) Cadeia efetiva obtida pela primeira abordagem do SDRG após uma varredura na cadeia.

As subsequentes varreduras dão origem a uma distribuição de acoplamentos autossimilar de acoplamentos que contém três parâmetros, como ilustra a figura 6.2, de onde obtemos o conjunto de equações recursivas dado por

$$
\begin{aligned}
J_{1}^{(j+1)} & =\left(\frac{4}{3}\right) \frac{J_{2}^{(j)} J_{3}^{(j)}}{J_{1}^{(j)}}, \quad J_{2}^{(j+1)}=\left(\frac{4}{3}\right)^{2} \frac{J_{2}^{(j)}\left[J_{3}^{(j)}\right]^{2}}{\left[J_{1}^{(j)}\right]^{2}}, \\
J_{3}^{(j+1)} & =\left(\frac{4}{3}\right)^{3} \frac{J_{2}^{(j)}\left[J_{3}^{(j)}\right]^{3}}{\left[J_{1}^{(j)}\right]^{3}}
\end{aligned}
$$

que são válidas se $J_{1}^{(j)}>J_{2}^{(j)}$. Esta última condição é sempre válida se $J_{b}>(4 / 3) J_{a}$.

Definindo duas razões entre os acoplamentos $J_{1}, J_{2}$ e $J_{3}$, podemos escrever as equações de recorrência

$$
\begin{aligned}
\rho^{(j+1)} & \equiv \frac{J_{1}^{(j+1)}}{J_{3}^{(j+1)}}=\left[\frac{3}{4} \rho^{(j)}\right]^{2}, \\
\sigma^{(j+1)} & \equiv \frac{J_{1}^{(j+1)}}{J_{2}^{(j+1)}}=\frac{3}{4} \rho^{(j)},
\end{aligned}
$$




$$
-\begin{array}{lllll}
J_{1}^{(i)} \quad \cdots & J_{2}^{(i)} \quad \cdots J_{3}^{(i)}
\end{array}
$$

(a)

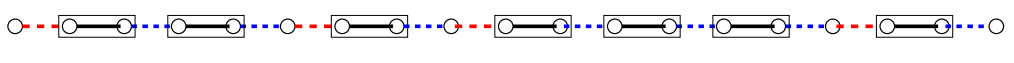

(b)

$$
J_{2}^{(i+1)} \quad J_{1}^{(i+1)} \quad J_{3}^{(i+1)}
$$

Figura 6.2: Distribuição autossimilar de acoplamentos obtida da primeira abordagem para as varreduras subsequentes à primeira. Singletos são formados entre os spins conectados pelo acoplamento forte $J_{1}^{(i)}$.

onde é claro que, se a condição $J_{b}>(4 / 3) J_{a}$ é satisfeita, há apenas um único ponto fixo de modulação infinita, $\rho^{\infty}=\sigma^{\infty}=\infty$. Assim, com a iteração do SDRG, alcançamos o estado fundamental sem encontrar nenhum gap, pois $J_{1}^{(i)}$, estimativa para o espectro da cadeia, se aproxima continuamente de zero. Ou seja, o gap de Haldane foi destruído. Portanto, a primeira abordagem prediz que, no limite de modulação forte, os acoplamentos seguindo a sequência 6-3 induzem uma transição de fase, para uma fase de singleto aperiódico sem gap, na qual a relação de escala é calculada na seção 6.4. Uma estimativa grosseira para o ponto crítico que separa a fase de Haldane da fase dominada pela aperiodicidade é dada pela condição $J_{b}>(4 / 3) J_{a}$.

\subsection{SDRG: Segunda abordagem.}

A figura 6.3 mostra os resultados da aplicação da segunda abordagem do SDRG para a cadeia de Heisenberg de spin 1 com acoplamentos modulados pela sequência 6-3. Na figura 6.3(b), todos os pares de spin conectados por $J_{b}$ são substituídos por pares de spin $1 / 2$ após a primeira varredura, e para $J_{b} \gtrsim 1.91 J_{a}$ todos os pares de spin $1 / 2$ são dizimados, levando à configuração na figura 6.3(c), com três acoplamentos efetivos dados por

$$
J_{1}^{(0)}=J_{a}, \quad J_{2}^{(0)}=\left(\frac{1}{2}\right) \frac{J_{a}^{2}}{J_{b}}, \quad J_{3}^{(0)}=\left(\frac{1}{2}\right)^{2} \frac{J_{a}^{3}}{J_{b}^{2}} .
$$




\section{... $J_{a} \cdot \operatorname{Spin} 1 / 2$ \\ $-J_{b} \circ$ Spin 1}

(a)

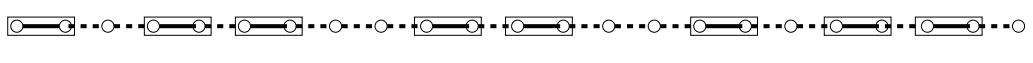

(b)

(c)

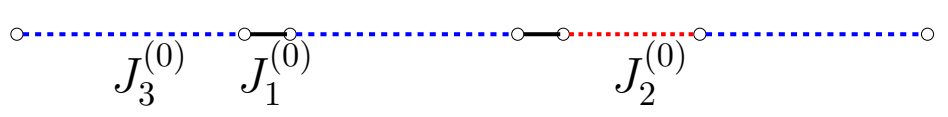

Figura 6.3: Primeiro passo da renormalização da cadeia de spin 1 com acoplamento seguindo a sequência 6-3 de acordo com a segunda abordagem. (a) A cadeia original (b) Pares de spin 1 conectados pelo acoplamento forte $J_{b}$ são substituídos por pares de spin $1 / 2$ (c) Pares de spin 1/2 são dizimados e dão origem a acoplamentos efetivos entre os spins 1 remanescentes.

Se recomeçamos da configuração na figura 6.3(c), cada passo subsequente do SDRG envolve duas varreduras consecutivas ao longo da cadeia, a primeira substituindo os pares de spin 1 conectados por $J_{1}$ por pares de spin $1 / 2$, os quais são então dizimados dando origem a novas ligações efetivas. Este processo está ilustrado na figura 6.4, o que gera as seguintes equações de recorrência

$$
\begin{aligned}
J_{1}^{(j+1)} & =\left(\frac{1}{2}\right) \frac{J_{2}^{(j)} J_{3}^{(j)}}{J_{1}^{(j)}}, \quad J_{2}^{(j+1)}=\left(\frac{1}{2}\right)^{2} \frac{J_{2}^{(j)}\left[J_{3}^{(j)}\right]^{2}}{\left[J_{1}^{(j)}\right]^{2}}, \\
J_{3}^{(j+1)} & =\left(\frac{1}{2}\right)^{3} \frac{J_{2}^{(j)}\left[J_{3}^{(j)}\right]^{3}}{\left[J_{1}^{(j)}\right]^{3}},
\end{aligned}
$$

nas quais $j$ designa o passo do SDRG. Estas equações são válidas se $J_{1}^{(j)}>\frac{3}{2} J_{2}^{(j)}$, condição que é sempre verificada para $J_{b} \gtrsim 1.91 J_{a}$.

Assim como fizemos na primeira abordagem, podemos definir as razões entre os acopla- 


$$
\begin{aligned}
& -J_{1}^{(i)} \\
& \text {-.- } J_{2}^{(i)} \bullet \operatorname{Spin} 1 / 2 \\
& \cdots J_{3}^{(i)} \circ \operatorname{Spin} 1
\end{aligned}
$$

(a)

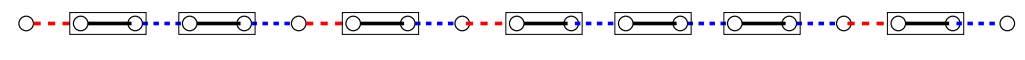

(b)

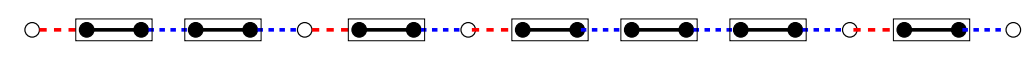

(c)

$$
J_{2}^{(i+1)} \quad J_{1}^{(i+1)} \quad J_{3}^{(i+1)}
$$

Figura 6.4: Distribuição autossimilar de acoplamentos obtida da segunda abordagem para os passos subsequentes do SDRG (a) Cadeia efetiva que consiste somente de spins 1 (b) Pares de spins 1 fortemente correlacionados dão origem a pares de spin 1/2. (c) Pares de spin 1/2 são dizimados e dão origem a novos parâmetros efetivos, novamente consistindo somente de spins 1, com uma distribuição de acoplamentos invariante.

mentos $\rho \equiv J_{1} / J_{3}$ e $\sigma \equiv J_{1} / J_{2}$, que têm como equações de recorrência

$$
\begin{aligned}
& \rho^{(j+1)}=\left[2 \rho^{(j)}\right]^{2}, \\
& \sigma^{(j+1)}=2 \rho^{(j)} .
\end{aligned}
$$

Estas também levam a um ponto fixo de modulação infinita, $\rho^{\infty}=\sigma^{\infty}=\infty$, então as previsões da primeira e segunda abordagens estão agora qualitativamente em acordo, porém, como ocorreu com a cadeia de Fibonacci-Heisenberg, os níveis de energia previstos pelas duas abordagens (estimados pelo acoplamento efetivo $J_{1}$ ) são quantitativamente diferentes.

Se $J_{b} \lesssim 1.91 J_{a}$, implementações numéricas da segunda abordagem não detalhadas aqui predizem uma sucessão diferente de processos de renormalização daquele encontrado no primeiro passo do SDRG, de acordo com o esquema que associa a escala de energia com o maior gap local dos blocos de spins. Mesmo assim, para $1.69 J_{a} \lesssim J_{b} \lesssim 1.91 J_{a}$, a distribuição de acoplamentos efetivos da figura 6.4(a) é eventualmente alcançada após poucos passos do SDRG, então o SDRG ainda prediz uma fase de singleto aperiódico sem gap como estado fundamental. 
Para $J_{b} \lesssim 1.69 J_{a}$, porém, a distribuição de acoplamentos e spins alcança eventualmente uma cadeia de spins $1 / 2$ dimerizada. É conhecido que a cadeia de spins $1 / 2$ dimerizada tem um estado fundamental com gap adiabaticamente ligado à fase de Haldane [44]. Assim, dentro das aproximações usadas para construir a segunda abordagem, $J_{b} \simeq 1.69 J_{a}$ corresponde ao ponto crítico que separa a fase com gap daquela sem gap dominada pela aperiodicidade.

\subsection{SDRG: Terceira abordagem.}

A figura 6.5 mostra o primeiro passo da renormalização da cadeia de spin 1 de Heisenberg com acoplamentos que seguem a sequência 6-3, de acordo com a terceira abordagem. Os pares de spin 1/2 são formados a partir de pares de spin 1 fortemente conectados, quando assumimos que $J_{b}>3 J_{a}$, e quando dizimados usando teoria de perturbação de segunda e terceira ordem, encontramos dois acoplamentos efetivos, juntamente com a ligação $J_{a}$ remanescente, que definem um conjunto de equações de recorrência dado por

$$
\begin{aligned}
& J_{1}^{(0)}=J_{a}, \\
& J_{2}^{(0)}=\frac{\left(\alpha_{+}-\alpha_{-}\right)^{2}}{2} \frac{J_{a}^{2}}{J_{b}}=\left(\frac{4}{3}\right) \frac{J_{a}^{2}}{J_{b}}, \\
& J_{3}^{(0)}=\frac{\left(\alpha_{+}-\alpha_{-}\right)^{4}}{4} \frac{J_{a}^{3}}{J_{b}^{2}}=\left(\frac{4}{3}\right)^{2} \frac{J_{a}^{3}}{J_{b}^{2}},
\end{aligned}
$$

que são exatamente as mesmas da primeira abordagem. Devemos notar que, como aconteceu na cadeia de Fibonacci-Heisenberg, ligações além de primeiros vizinhos são introduzidas no passo intermediário, porém eliminadas ao final do passo quando a modulação é forte. Além do mais elas são essenciais para que se obtenha a mesma previsão da primeira abordagem.

Os subsequentes passos do SDRG começando a partir da distribuição na figura 6.5)(c), são ilustrados na figura 6.6(a). Após a formação de pares de spin 1/2 a partir do pares de spin 1 fortemente acoplados por $J_{1}$, estes pares são dizimados, e levando em conta as ligações além de primeiros vizinhos, essa dizimação resulta, utilizando teoria de perturbação de segunda, terceira e quarta ordem, em novos acoplamentos efetivos que obe- 


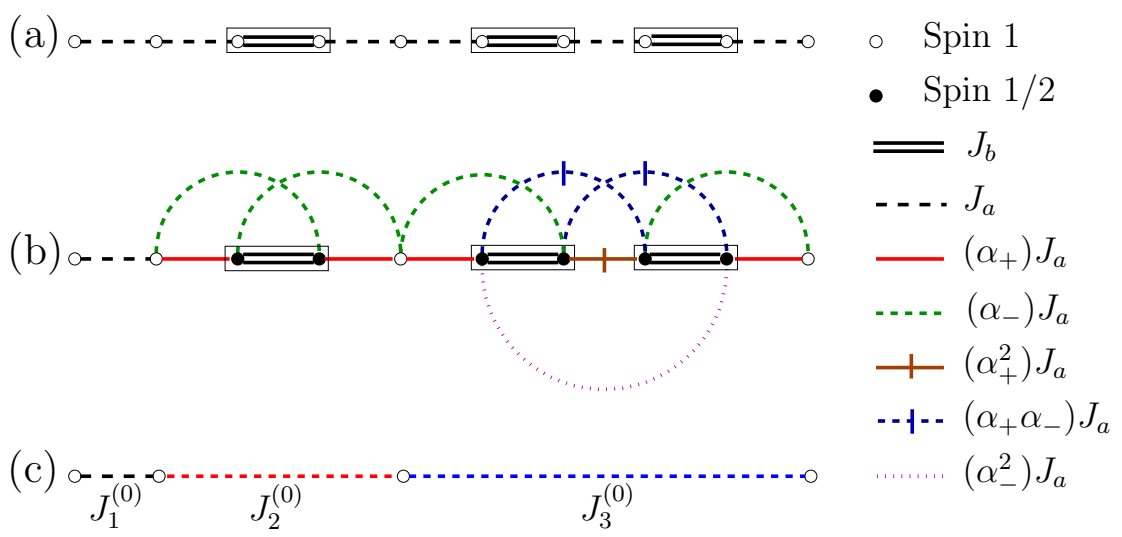

Figura 6.5: Primeiro passo do SDRG da cadeia de Heisenberg com acoplamentos segundo a sequência 6-3 de acordo com a terceira abordagem. (a) A cadeia original. (b) Pares de spin 1 conectados pela ligação mais forte $J_{b}$ são substituídos por pares de spin 1/2, ligações que conectam segundos ou terceiros vizinhos são criadas. (c) Pares de spin 1/2 são dizimados dando origem a acoplamentos efetivos entre os spins 1 remanescentes.

decem as equações de recorrência

$$
\begin{aligned}
J_{1}^{(j+1)} & =\frac{\left(\alpha_{+}-\alpha_{-}\right)^{2}}{2} \frac{J_{2}^{(j)} J_{3}^{(j)}}{J_{1}^{(j)}}=\left(\frac{4}{3}\right) \frac{J_{2}^{(j)} J_{3}^{(j)}}{J_{1}^{(j)}} \\
J_{2}^{(j+1)} & =\frac{\left(\alpha_{+}-\alpha_{-}\right)^{4}}{4} \frac{J_{2}^{(j)}\left[J_{3}^{(j)}\right]^{2}}{\left[J_{1}^{(j)}\right]^{2}}=\left(\frac{4}{3}\right)^{2} \frac{J_{2}^{(j)}\left[J_{3}^{(j)}\right]^{2}}{\left[J_{1}^{(j)}\right]^{2}}, \\
J_{3}^{(j+1)} & =\frac{\left(\alpha_{+}-\alpha_{-}\right)^{6}}{8} \frac{J_{2}^{(j)}\left[J_{3}^{(j)}\right]^{3}}{\left[J_{1}^{(j)}\right]^{3}}=\left(\frac{4}{3}\right)^{3} \frac{J_{2}^{(j)}\left[J_{3}^{(j)}\right]^{3}}{\left[J_{1}^{(j)}\right]^{3}},
\end{aligned}
$$

que são válidas somente se $J_{b}>\alpha_{+}^{2} J_{a}$.

Assim, para modulação suficientemente forte, a primeira e terceira abordagens dão o mesmo resultado qualitativo para as propriedades do estado fundamental e também os mesmo níveis de energia, enquanto a segunda abordagem concorda apenas qualitativamente com as outras abordagens.

$\mathrm{Na}$ presença de modulação moderada ou fraca, na qual os cálculos de teoria de perturbação são inadequados, predições quantitativas dependem de detalhes sutis das primeiras e terceiras abordagens. De fato, para $\alpha_{+}^{2} J_{a}<J_{b}<3 J_{a}$, implementações 


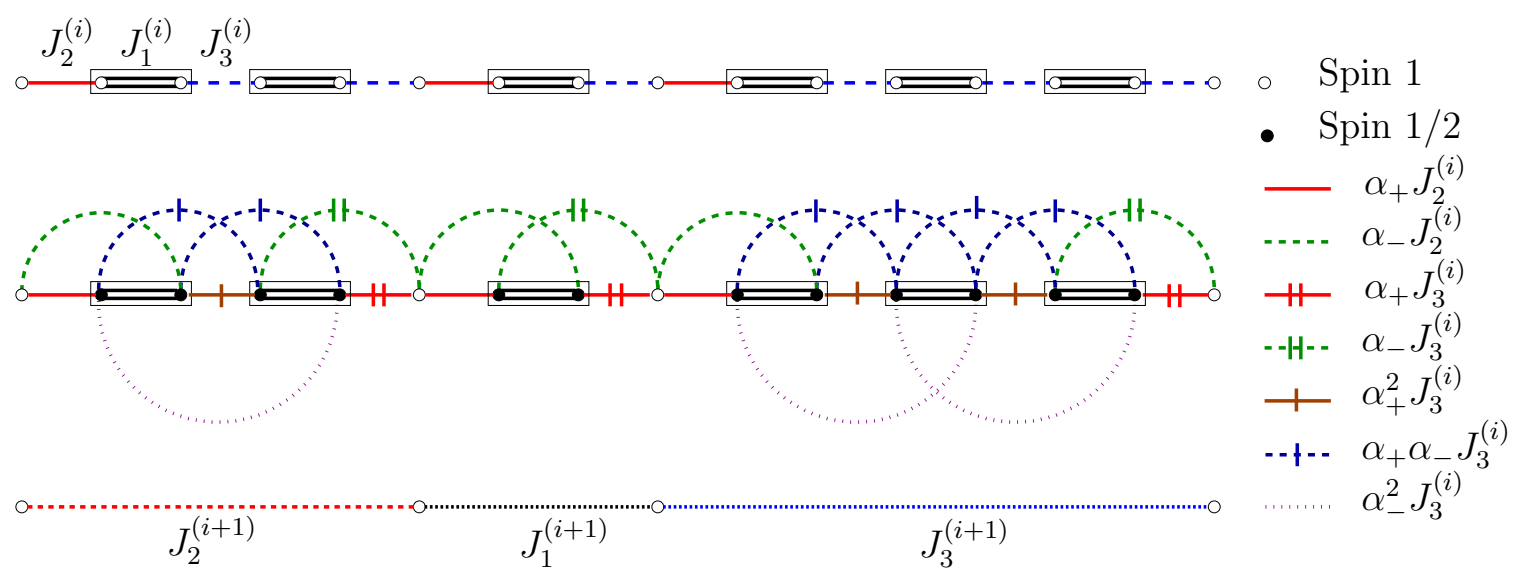

Figura 6.6: Distribuição autossimilar de acoplamentos obtida obtida da terceira abordagem nos passos subsequentes do SDRG. (a) Cadeia efetiva constituída apenas por spins 1 (b) Pares de spin 1 fortemente conectados por $J_{1}$ forma pares de spin $1 / 2$, e ligações além dos primeiros vizinhos são criadas. (c) Pares de spin 1/2 são dizimados, dando origem a novos acoplamentos efetivos, os quais novamente conectam somente spins 1, com uma distribuição de acoplamentos invariante.

numéricas da terceira abordagem ainda predizem um estado fundamental sem gap, porém com pequenas diferenças nos primeiros passos do processo de renormalização. Porém, para $J_{b} \lesssim \alpha_{+}^{2} J_{a}$, a terceira abordagem eventualmente mostra uma cadeia de spins $1 / 2$ dimerizada, mostrando assim uma fase com gap. Claramente, para o intervalo de parâmetros considerado, não esperamos que tal predição para o ponto crítico seja precisa.

\subsection{Relação dinâmica de escala.}

No limite de modulação forte, sem gap, podemos derivar a relação de escala dinâmica entre escalas de energia e comprimento. É natural assumirmos que, como os níveis de energia são estimados a partir do valor do acoplamento efetivo mais forte em cada passo do SDRG, a escala de comprimento relevante é o correspondente comprimento efetivo. A partir da relação de recorrência (6.11), e da figura 6.6. podemos ver que os comprimentos dos acoplamentos efetivos satisfazem uma relação de recorrência que pode ser escrita em 
uma forma matricial como

$$
\left[\begin{array}{l}
\ell_{1}^{(j+1)} \\
\ell_{2}^{(j+1)} \\
\ell_{3}^{(j+1)}
\end{array}\right]=\left[\begin{array}{lll}
1 & 1 & 1 \\
2 & 1 & 2 \\
3 & 1 & 3
\end{array}\right] \cdot\left[\begin{array}{c}
\ell_{1}^{(j)} \\
\ell_{2}^{(j)} \\
\ell_{3}^{(j)}
\end{array}\right],
$$

onde novamente $j$ designa os passos do SDRG. A matriz que aparece na equação acima tem autovalores $\lambda_{1}=\lambda_{2}=0$ e $\lambda_{3}=5$, de modo que, no limite assintótico, todas as escalas de comprimento são dadas por

$$
\ell^{(j)} \sim \lambda_{3}^{j}
$$

Os níveis de energia, sendo proporcionais ao valor do maior acoplamento em cada iteração, escalam como $\Delta_{j} \sim J_{1}^{(j)}$. Assim, resolvendo a relação de recorrência nas equações (6.5) e (6.11), podemos escrever

$$
J_{1}^{(j+1)}=\frac{4}{3}\left[\rho^{(j)}\right]^{-\frac{3}{2}} J_{1}^{(j)}
$$

Para a razão $\rho^{(j)}$ podemos iterar a equação (6.5) e obter

$$
\rho^{(j)}=\exp \left[2^{j} \ln \rho^{(0)}-2 \ln (4 / 3)\left(2^{j}-1\right)\right]
$$

e usando este resultado na equação recursiva para o acoplamento mais forte encontramos

$$
J_{1}^{(j)}=(4 / 3)^{j} \exp \left[-\frac{3}{2} \ln \left(\rho^{(0)}(4 / 3)^{-2}\right)\left(2^{j}-1\right)-3 j \ln (4 / 3)\right] .
$$

Finalmente usando o último resultado juntamente com a equação (6.12) para eliminar a dependência em $j$ obtemos a relação de escala dinâmica

$$
\Delta_{j} \sim \ell_{j}^{\frac{-2 \ln (4 / 3)}{\ln 5}} \exp \left[-\frac{3}{2} \ln \left(\frac{9}{16} \rho^{(0)}\right) \ell_{j}^{\omega}\right]
$$

$\operatorname{com} \omega=\frac{\ln 2}{\ln 5}$.

Como esperado, desconsiderando uma constante não importante, esta é a mesma forma exponencial obedecida pela cadeia de Heisenberg de spin 1/2 com acoplamentos que seguem a sequência 6-3. 



\section{Capítulo 7}

\section{Simulações de Monte Carlo quântico.}

\subsection{O método de Monte Carlo quântico.}

Nesta seção descreveremos resumidamente o método de Monte Carlo quântico. Leitores familiarizados com o tema podem iniciar a leitura na seção 7.2. onde expomos os resultados obtidos das simulações das cadeias de Heisenberg aperiódicas. Trataremos esse tema de forma qualitativa, detalhes técnicos podem ser encontrados nas referências inseridas. Essa parte do texto foi baseada na referência [45]. Na primeira seção mostramos uma representação da função de partição, a partir de uma expansão em série de Taylor do peso de Boltzmann, apropriada para a construção de um espaço configuracional (amostra) que será usado no método de Monte Carlo. Na seção seguinte escolhemos o modelo de Heisenberg de spin 1/2 para exemplificar como as atualizações na amostra (updates) podem ser construídos no espírito do algoritmo de Metropolis.

\subsubsection{Representação em série de Taylor da função de partição.}

Comumente chamado de método de SSE [46] (stochastic series expansion, ou expansão estocástica em série), este método consiste em encontrar uma maneira de representarmos os termos da soma na função de partição

$$
\mathrm{Z}=\operatorname{Tr}\left\{\mathrm{e}^{-\beta \mathcal{H}}\right\}
$$


de forma apropriada para as simulações de Monte Carlo, ou seja, queremos representar os operadores e estados presentes na função de partição de forma numerável e apropriada para simulações numéricas.

Partimos de uma expansão em série de Taylor do peso de Boltzmann para a construção das configurações que serão usadas nos cálculos de Monte Carlo,

$$
\mathrm{e}^{-\beta \mathcal{H}}=\sum_{n=0}^{\infty} \frac{(-\beta)^{n}}{n !} \mathcal{H}^{n}
$$

Esta abordagem é fortemente relacionada com as primeiras abordagens em simulações de Monte Carlo quântico utilizando-se integrais de trajetória [45].

Escolhendo uma base apropriada, e inserindo um conjunto completo de estados entre cada operador, podemos reescrever a função de partição como

$$
\mathrm{Z}=\sum_{n=0}^{\infty} \frac{(-\beta)^{n}}{n !} \sum_{\{\alpha\}_{n}}\left\langle\alpha_{0}|\mathcal{H}| \alpha_{n-1}\right\rangle \cdots\left\langle\alpha_{2}|\mathcal{H}| \alpha_{1}\right\rangle\left\langle\alpha_{1}|\mathcal{H}| \alpha_{0}\right\rangle
$$

onde $\{\alpha\}_{n}$ indica que devemos somar sobre $n$ estados.

Para construirmos uma configuração de tamanho fixo, onde queremos que todos os termos da soma tenham o mesmo número de operadores, truncamos a soma em $n=L$, e aumentamos o números de operadores em cada elemento da soma com $L-n$ operadores identidade I. Se permitimos que em cada elemento os operadores identidades ocupem todas as possíveis posições, podemos definir um operador string $\mathcal{S}=\mathcal{S}_{1}, \mathcal{S}_{2}, \ldots, \mathcal{S}_{L}$, para o qual $\mathcal{S}_{i} \in\{\mathcal{H}, \mathrm{I}\}$. Se somarmos sobre todas essas sequências, ponderando cada uma delas por $\left(\begin{array}{l}L \\ n\end{array}\right)$, que é o número de termos iguais adicionados, podemos reescrever a função de partição como

$$
\mathrm{Z} \simeq \sum_{\mathcal{S}} \frac{(-\beta)^{n}(L-n) !}{L !} \sum_{\{\alpha\}_{L}} \sum_{\left\{\mathcal{S}_{i}\right\}}\left\langle\alpha_{0}\left|\mathcal{S}_{L}\right| \alpha_{L-1}\right\rangle \cdots\left\langle\alpha_{2}\left|\mathcal{S}_{2}\right| \alpha_{1}\right\rangle\left\langle\alpha_{1}\left|\mathcal{S}_{1}\right| \alpha_{0}\right\rangle
$$

onde $n$ representa o número de elementos para os quais $\mathcal{S}_{i}=\mathcal{H}$ no operador string e a soma em $\left\{\mathcal{S}_{i}\right\}$ contém exatamente $n$ termos iguais a $\mathcal{H}$. A soma em $n$ está implícita na soma em $\mathcal{S}$, que é uma soma onde há um número maior de operadores $\mathcal{S}_{i}=\mathcal{H}$ em cada termo, começando de um único operador.

Na próxima seção definiremos um modelo e mostraremos em maior detalhe como a par- 
tir da última equação podemos construir uma configuração adequada para as simulações de Monte Carlo. Agora é interessante calcular o valor médio da energia. De acordo com a função de partição (17.3) temos

$$
E=\sum_{n=0}^{\infty} \frac{(-\beta)^{n}}{n !} \sum_{\{\alpha\}_{n+1}}\left\langle\alpha_{0}|\mathcal{H}| \alpha_{n}\right\rangle \cdots\left\langle\alpha_{2}|\mathcal{H}| \alpha_{1}\right\rangle\left\langle\alpha_{1}|\mathcal{H}| \alpha_{0}\right\rangle .
$$

Essa soma pode ser rearranjada na forma

$$
E=-\sum_{n=1}^{\infty} \frac{(-\beta)^{n}}{n !} \frac{n}{\beta} \sum_{\{\alpha\}_{n}}\left\langle\alpha_{0}|\mathcal{H}| \alpha_{n}\right\rangle \cdots\left\langle\alpha_{2}|\mathcal{H}| \alpha_{1}\right\rangle\left\langle\alpha_{1}|\mathcal{H}| \alpha_{0}\right\rangle
$$

onde identificamos novamente os termos da função de partição, assim concluímos que

$$
E=-\frac{\langle n\rangle}{\beta}
$$

Esse resultado nos mostra que embora a soma em $n$ na função de partição se estenda ao infinito, a distribuição se anula exponencialmente para valores de $n$ maiores que um valor certo finito, tipicamente $n_{\max } \propto N \beta$, pois $E \propto N$. Assim, podemos afirmar que o truncamento para $L$ finito, devidamente determinado, não causa erros mensuráveis e não deve ser considerado uma aproximação.

\subsubsection{O método de SSE para o modelo de Heisenberg de spin $1 / 2$.}

Mostramos agora como implementar o método de Monte Carlo quântico SSE utilizando o modelo de Heisenberg de spin 1/2 como exemplo. Escolhemos uma notação que não especifica a rede onde o modelo está definido, escrevendo a hamiltoniana como uma soma de operadores que atuam nos pares de spins,

$$
\mathcal{H}=\sum_{b=1}^{N_{b}} \mathcal{H}_{b}, \quad \text { onde } \quad \mathcal{H}_{b}=J_{b} \mathbf{S}_{i(b)} \cdot \mathbf{S}_{j(b)},
$$

onde somamos sobre uma lista de pares de sítios acoplados $[i(b), j(b)], \operatorname{com} b=1,2, \ldots, N_{b}$. Inicialmente consideramos $J_{b}=J>0$, sistema uniforme. Adaptações para um caso não 
uniforme podem ser feitas sem grandes dificuldades.

Dividimos a interação em uma parte diagonal e outra parte não diagonal, escolhendo como base os autoestados de $S^{z}$. Definimos os operadores $\mathcal{H}_{a, b}$, com $a=1,2$, representando a parte diagonal e a parte não diagonal da hamiltoniana,

$$
\begin{aligned}
\mathcal{H}_{1, b} & =\frac{1}{4}-S_{i(b)}^{z} S_{j(b)}^{z}, \\
\mathcal{H}_{2, b} & =\frac{1}{2}\left(S_{i(b)}^{+} S_{j(b)}^{-}+S_{i(b)}^{-} S_{j(b)}^{+}\right) .
\end{aligned}
$$

Repare que introduzimos uma constante e um sinal negativo na parte diagonal, garantindo que $\mathcal{H}_{1, b}>0$, para que a expansão seja positiva definida, assim a hamiltoniana pode ser escrita como

$$
\mathcal{H}=-J \sum_{b=1}^{N_{b}}\left(\mathcal{H}_{1, b}-\mathcal{H}_{2, b}\right)+\frac{J N_{b}}{4}
$$

onde a constante $J N_{b} / 4$ pode ser negligenciada, sendo necessária apenas para o cálculo da energia total.

Inicialmente escrevemos a função de partição na forma da equação (7.3) escrevendo todas as potências de $\mathcal{H}$ como um produto dos operadores de pares que acabamos de introduzir. Assim o ponto de partida para construir um algoritmo de Monte Carlo quântico, por meio da abordagem de SSE, para modelo de Heisenberg de spin $1 / 2$ é a equação

$$
\begin{aligned}
\mathrm{Z}=\sum_{\{\alpha\}_{n}} \sum_{n=0}^{\infty}(-1)^{n_{2}} \frac{\beta^{n}}{n !} \sum_{\mathcal{S}_{n}}\left\langle\alpha_{0}\left|\mathcal{H}_{a(p), b(p)}\right| \alpha_{n-1}\right\rangle \cdots \\
\cdots\left\langle\alpha_{2}\left|\mathcal{H}_{a(p), b(p)}\right| \alpha_{1}\right\rangle\left\langle\alpha_{1}\left|\mathcal{H}_{a(p), b(p)}\right| \alpha_{0}\right\rangle,
\end{aligned}
$$

onde redefinimos o inverso da temperatura $\beta=J / T$ de modo a absorver a constante $J$. Nessa forma o operador string $\mathcal{S}_{n}$ se refere a uma lista de operadores de pares, equações (7.9), gerados pelas potências da hamiltoniana $\mathcal{H}^{n}$,

$$
\mathcal{S}_{n}=[a(0), b(0)],[a(1), b(1)], \ldots,[a(n-1), b(n-1)],
$$

e $n_{2}$ é o numero total de de operadores não diagonais, ou seja, o números de elementos com $a(i)=2$ no operador string. A soma sobre o operador string $\mathcal{S}_{n}$ cobre todos os 
índices $a=1,2$ e $b=1,2, \ldots, N_{b}$ que especificam a rede onde o modelo está definido.

Quando o operador string atua em um estado da base $|\alpha\rangle=\left|S_{1}^{z}, \ldots, S_{N}^{z}\right\rangle$, obtemos uma evolução desse estado para um outro estado dessa base. Esse estado será chamado de estado propagado,

$$
|\alpha(p)\rangle \propto \prod_{i=0}^{p-1} \mathcal{H}_{a(i), b(i)}|\alpha\rangle .
$$

Com a introdução da constante 1/4 nos operadores diagonais, todas as operações em estados com spins paralelos destroem o estado,

$$
\begin{array}{rlrl}
\mathcal{H}_{1, b}\left|\uparrow_{i(b)} \uparrow_{j(b)}\right\rangle=0, & \mathcal{H}_{2, b}\left|\uparrow_{i(b)} \uparrow_{j(b)}\right\rangle=0, \\
\mathcal{H}_{1, b}\left|\downarrow_{i(b)} \downarrow_{j(b)}\right\rangle & =0, & & \mathcal{H}_{2, b}\left|\downarrow_{i(b)} \downarrow_{j(b)}\right\rangle=0,
\end{array}
$$

enquanto todas as operações em pares de spins anti-paralelos dão o mesmo resultado,

$$
\begin{aligned}
\left\langle\uparrow_{i(b)} \downarrow_{j(b)}\left|\mathcal{H}_{1, b}\right| \uparrow_{i(b)} \downarrow_{j(b)}\right\rangle & =\frac{1}{2}, & \left\langle\downarrow_{i(b)} \uparrow_{j(b)}\left|\mathcal{H}_{2, b}\right| \uparrow_{i(b)} \downarrow_{j(b)}\right\rangle & =\frac{1}{2}, \\
\left\langle\downarrow_{i(b)} \uparrow_{j(b)}\left|\mathcal{H}_{1, b}\right| \downarrow_{i(b)} \uparrow_{j(b)}\right\rangle & =\frac{1}{2}, & \left\langle\uparrow_{i(b)} \downarrow_{j(b)}\left|\mathcal{H}_{2, b}\right| \downarrow_{i(b)} \uparrow_{j(b)}\right\rangle & =\frac{1}{2} .
\end{aligned}
$$

Assim, uma configuração do operador-estado $\left(\alpha, \mathcal{S}_{L}\right)$ dá uma contribuição não nula para a função de partição $\mathcal{S}_{L}$, quando envolve apenas operações em pares de spins antiparalelos. Além desse vínculo local, nas configurações que contribuem para a função de partição, o estado propagado deve satisfazer condições periódicas de contorno $|\alpha(L-1)\rangle=|\alpha(0)\rangle$.

Note que, enquanto o operador $\mathcal{H}_{1, b}$ tem contribuições não nulas somente entre estados iguais, para o operador $\mathcal{H}_{2, b}$, as contribuições não nulas ocorrem entre estados com spins invertidos.

Podemos agora truncar a soma na função de partição para construir uma amostra configuracional de tamanho fixo. Truncaremos a soma em $L$ e deixaremos a discussão sobre como determinar $L$ para depois. Na prática $L$ será determinado pelo próprio algoritmo de Monte Carlo. Devemos lembrar que o truncamento da soma não causa nenhum erro relevante nas simulações. Definimos o operador identidade como $\mathcal{H}_{0,0}=\mathrm{I}$, que será usado para aumentar a ordem dos termos da soma, assim o elemento $[a(0), b(0)]=[0,0]$ passa a fazer parte das configurações permitidas na lista de índices da operador string. Assim a 
função de partição pode ser escrita como

$$
\begin{gathered}
\mathrm{Z}=\sum_{\{\alpha\}_{n}} \sum_{\mathcal{S}_{L}}(-1)^{n_{2}} \frac{\beta^{n}(L-n) !}{L !}\left\langle\alpha_{0}\left|\mathcal{H}_{a(p), b(p)}\right| \alpha_{n-1}\right\rangle \cdots \\
\cdots\left\langle\alpha_{2}\left|\mathcal{H}_{a(p), b(p)}\right| \alpha_{1}\right\rangle\left\langle\alpha_{1}\left|\mathcal{H}_{a(p), b(p)}\right| \alpha_{0}\right\rangle,
\end{gathered}
$$

onde novamente para simplificar a notação, deixamos de lado os estados intermediários que havíamos introduzido. Agora $n$ se refere ao número de elementos diferentes de $[0,0]_{p}$ no operador string de tamanho fixo $\mathcal{S}_{L}$, e a soma em $n$ é feita de maneira implícita pela soma em $\mathcal{S}_{L}$.

Após a introdução dos estados intermediários, tipicamente, teremos elementos de matriz do tipo $\left\langle\alpha_{l+1}\left|\mathcal{H}_{a(p), b(p)}\right| \alpha_{l}\right\rangle$, onde $a(p)=0,1,2$. Portanto os termos não nulos na função de partição envolvem estados iguais $\left|\alpha_{l+1}\right\rangle=\left|\alpha_{l}\right\rangle$, quando $a(p)=0,1$, ou estados $\left|\alpha_{l+1}\right\rangle$ e $\left|\alpha_{l}\right\rangle$ com os spins $[i(b), j(b)]$ invertidos, quando $a(p)=2$, devido aos elementos de matriz não nulos da equação (7.15).

A figura 7.1 ilustra uma configuração típica de um termo não nulo da somatória que contribui para a função de partição. Os spins com $S^{z}=-1 / 2$ são representados por círculos preenchidos, enquanto os spins $S^{z}=+1 / 2$ são representados por círculos vazios. Os operadores diagonais são representados por barras vazias, enquanto os operadores não diagonais são representados por barras preenchidas. Repare que os operadores diagonais ocorrem somente entre pares paralelos na direção $p$ (vertical), enquanto os operadores não diagonais ocorrem somente entre pares de spins anti-paralelos nesta mesma direção. A direção $p$ pode ser comparada com a dimensão de tempo imaginário que ocorre em outras abordagens do método de Monte Carlo quântico [45], ou seja, há uma dimensão adicional nas simulações de Monte Carlo. Nessa configuração pode-se identificar caminhos formados pelos spins de mesma orientação. Note também as condições periódicas de contorno.

Como todos os elementos de matriz dos operadores de pares são iguais a $1 / 2$, o peso de uma configuração não nula é dado por

$$
W\left(\alpha, \mathcal{S}_{L}\right)=\left(\frac{\beta}{2}\right)^{n} \frac{(L-n) !}{L !}
$$

onde descartamos o fator $(-1)^{n_{2}}$, que é sempre positivo para qualquer rede bipartite, ou seja, para um sistema não frustrado. Isso se deve ao fato de que necessitamos de 


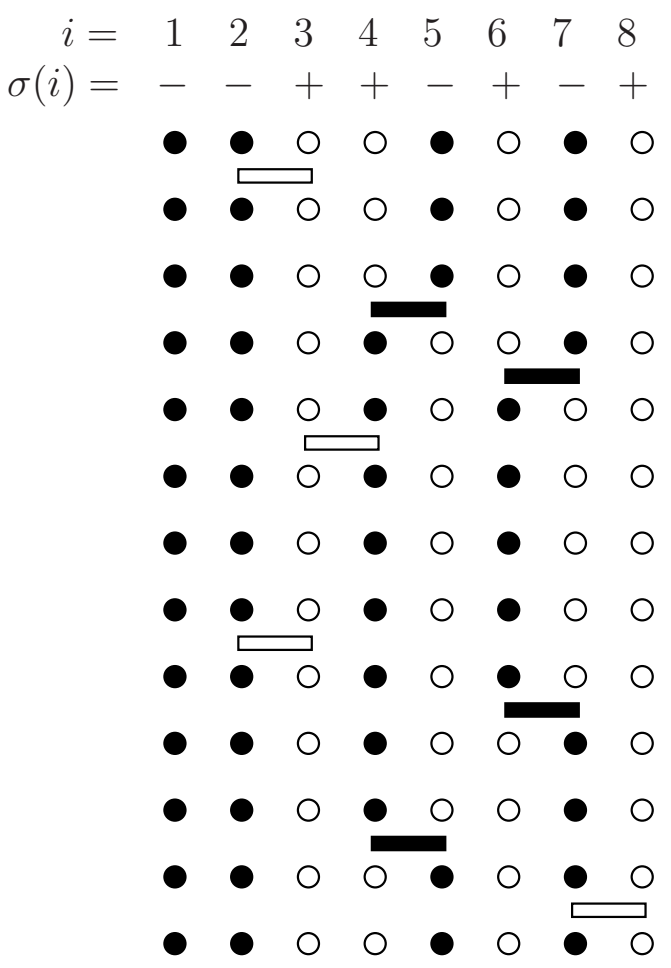

Figura 7.1: Configuração do método de Monte Carlo SSE para uma cadeia de 8 spins mostrando o estado propagado (direção vertical). Círculos vazios (preenchidos) representam spins $\sigma=+\frac{1}{2}\left(\sigma=-\frac{1}{2}\right)$. Barras vazias (preenchidas) representam operadores diagonais (não diagonais). A ausência de barras indica a presença de um operador identidade. A direção vertical $p$ é a direção do estado propagado. Entre duas linhas horizontais de spins há um estado $|\alpha(p)\rangle$ introduzido na equação (7.3).

um número par de operadores não diagonais, que alteram o caminho na direção $p$, para satisfazer as condições periódicas de contorno nessa direção, figura 7.1 .

A figura 7.1 representa a amostra que será usada para a simulação de Monte Carlo. Como é impraticável somarmos sobre todas as possíveis configurações do operador string que aparecem na soma do função de partição, torna-se necessário construirmos uma amostragem, ou um subconjunto, relevante em dada temperatura, dessas configurações para o cálculo de propriedades termodinâmicas do sistema em questão. Na próxima seção mostramos como atualizar essa amostra de acordo com o critério de balanceamento detalhado. 
Atualizações na amostra de Monte Carlo.

Após construir o espaço configuracional (amostra) usado nas simulações de Monte Carlo para a hamiltoniana (7.16), figura 7.1, precisamos encontrar uma maneira de atualizar essa amostra, ou fazer updates, completando assim o método de simulação. As atualizações na amostra alteram o operador $\mathcal{S}_{L}$ e o estado $|\alpha\rangle$. Deve-se notar que essas mudanças não são necessariamente independentes, como ilustra a figura 7.2, os operadores destacados por uma linha continua tem seus tipos $a=\{1,2\}$ alterados, figura $\mathbf{7 . 2}$ (a), enquanto os operadores destacados por uma linha pontilhada são alterados somente se o estado $|\alpha\rangle=|\alpha(0)\rangle$ também é alterado, figura 7.2 (b). Note que, em geral, precisamos de dois ou mais operadores para efetuar a mudança no tipo do operador.

(a)

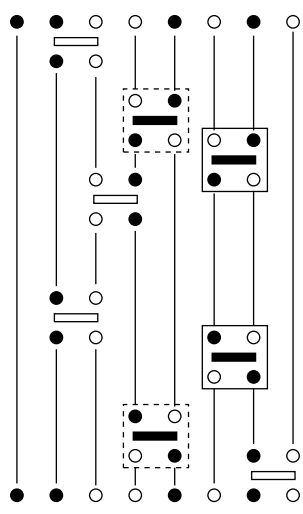

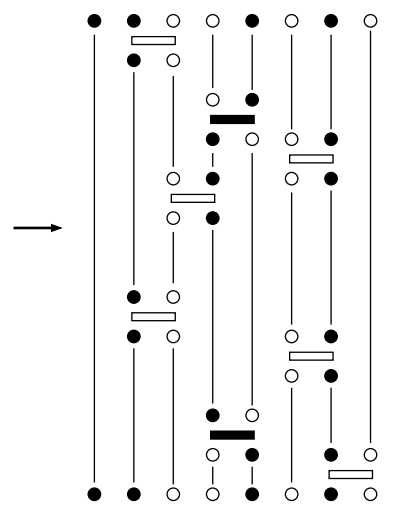

(b)

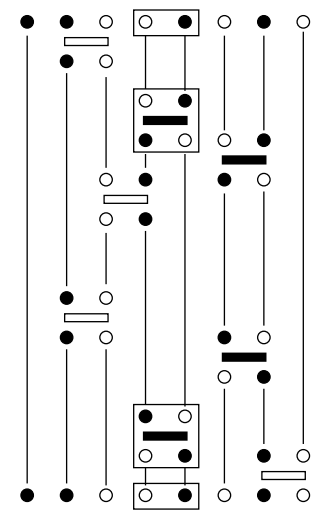

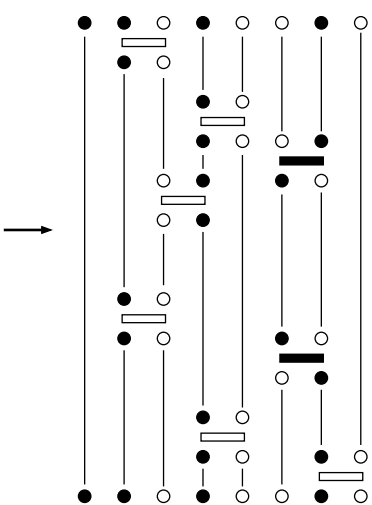

Figura 7.2: (a) Dois operadores não diagonais destacados por uma linha contínua tem seu tipo alterado para operadores diagonais, quando a orientação dos spins entre esses operadores é invertida. Tal atualização não pode ser feita nos operadores destacados por uma linha pontilhada. (b) Esses mesmo operadores, agora destacados por uma linha contínua, podem ser atualizados se o estado $|\alpha(0)\rangle$ e os spins em lados opostos aos operadores são alterados ao mesmo tempo. Nessa figura o spins com valores constantes foram substituídos por linhas verticais.

A estratégia para as simulações é construir uma amostra inicial com $n$ operadores diferentes da identidade, onde $[a, b]_{p} \neq[0,0]_{p}$, distribuídos aleatoriamente, $p \in\{0, \ldots, L-$ $1\} ; b=\left\{1, \ldots N_{b}\right\} ; a \in\{1,2\}$ em $\mathcal{S}_{L}$. Depois atualizamos essa amostra permitindo que $n$ seja alterado somente pela introdução ou exclusão de operadores diagonais $[0,0]_{p} \leftrightarrow[a, b]_{p}$, quando $n \rightarrow n \pm 1$. Finalmente escolhemos um subconjunto de operadores para terem seus tipos alterados $[1, b]_{p} \leftrightarrow[2, b]_{p}$. Esse conjunto de atualizações deve garantir que a amostragem de Monte Carlo seja ergódica, ou seja, cada configuração diferente pode ser 
alcançada com um número finito de atualizações.

Discutimos agora as atualizações dos operadores assumindo que a o valor de corte $n=L$ (tamanho da amostra) foi previamente determinado. Na prática o valor de $L$ é determinado pelo algoritmo exigindo-se que $L$ seja sempre maior do que o maior $n$ utilizado. Nas regras a seguir veremos como introduzir e retirar operadores de acordo com o princípio de balanceamento detalhado, alterando assim o valor de $n$. Então, um algoritmo de Monte Carlo quântico pode inicialmente escolher um $L>n$, e atualizar, ou aumentar, o valor de $L$ após cada passo do Monte Carlo de acordo com o aumento de $n$. Esse ponto ficará mais claro a seguir.

\section{Operadores diagonais.}

A atualização dos operadores diagonais no operador $\mathcal{S}_{L}$, como ilustra a figura 7.3, pode ser feita sequencialmente em $p \in\{0, \ldots, L-1\}$ e em posições aleatórias em $b=\left\{1, \ldots N_{b}\right\}$. Não há nenhum restrição na atualização $[1, b]_{p} \rightarrow[0,0]_{p}$, enquanto no caso oposto $[0,0]_{p} \rightarrow$ $[1, b]_{p}$ a inserção deve ocorrer entre spins que são antiparalelos, ou seja, devemos ter $\sigma(i(b)) \neq \sigma(j(b))$.

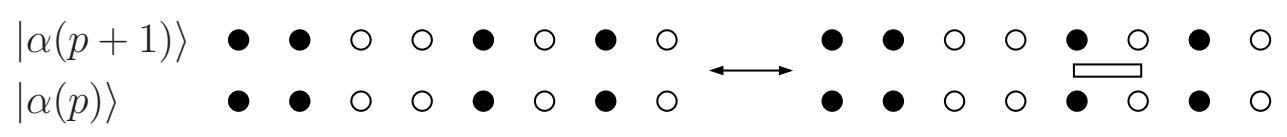

Figura 7.3: Inclusão ou retirada de um operador diagonal $[0,0]_{p} \leftrightarrow[1, b]_{p}$, em um acoplamento escolhido aleatoriamente. Enquanto não há restrições na retirada de um operador diagonal, na inserção os spins devem ser antiparalelos.

A probabilidade de aceitação de uma dessas atualizações pode ser obtida, no mesmo espirito da abordagem de Metrópolis, a partir da equação (7.17). No algoritmo de Metrópolis, a probabilidade de aceitação é escolhida como a probabilidade de sortear um determinado sítio, multiplicado pelo probabilidade de atualizar a configuração 1 do sistema

$$
P\left(C \rightarrow C^{\prime}\right)=P_{\text {seleção }}(i) P_{\text {aceitação }}\left(C^{\prime}\right),
$$

onde a probabilidade de aceitar a atualização é obtida dos pesos das configurações anterior

\footnotetext{
${ }^{1}$ Denotaremos genericamente a configuração do sistema por $C$
} 
e posterior à atualização, na forma

$$
P_{\text {aceitação }}\left(C^{\prime}\right)=\min \left[\frac{W\left(C^{\prime}\right)}{W(C)}, 1\right]
$$

onde $W\left(C^{\prime}\right)$ é o peso da configuração atualizada. Pode-se notar que se a configuração atualizada tiver um peso maior que a anterior, a atualização é aceita automaticamente.

Usando a equação (7.17) na equação anterior, e levando em conta que a inserção de um operador diagonal pode ocorrer em qualquer ponto ao longo da cadeia $\left\{1, \ldots, N_{b}\right\}$, enquanto a retirada de um operador pode ocorrer somente em um ponto da cadeia, encontramos as seguintes probabilidades:

$$
\begin{aligned}
& P([0,0] \rightarrow[1, b])=\min \left[\frac{\beta N_{b}}{2(L-n)}, 1\right], \\
& P([1, b] \rightarrow[0,0])=\min \left[\frac{2(L-n+1)}{\beta N_{b}}, 1\right] .
\end{aligned}
$$

Note que no caso $[0,0] \rightarrow[1, b]$, se os spins tiverem uma configuração $\sigma(i(b))=\sigma(j(b))$, a atualização é automaticamente rejeitada. Essas probabilidades satisfazem ao critério do balanceamento detalhado.

A partir das equações acima, podemos ver que há um $L$ ótimo que equilibra a inserção e exclusão dos operadores diagonais, repare na dependência em $L$ nas duas equações. Assim a amostra configuracional deve ter um tamanho $L$ aproximadamente constante, que deve ser alcançado durante a termalização.

\section{Operadores não diagonais, loops.}

Fica claro pela figura [7.2, que atualizações de operadores não diagonais devem envolver pelo menos dois operadores, para assim preservar o vínculo entre os caminhos formados por spins de mesmo valor, como os da figura 7.1, e os operadores distribuídos ao longo da amostra. Essas atualizações também devem satisfazer ao vínculo imposto pela condição periódica de contorno.

Uma maneira eficiente de realizar tais atualizações é ilustrado na figura 7.4, onde mostramos uma operação associada a um loop. Altera-se a orientação de todos os spins situados nos vértices do loop, assim como o tipo dos operadores que atuam nos spins, $[1, b] \leftrightarrow[2, b]$. Operadores tocados pelo loop duas vezes mantêm o mesmo tipo, e têm 

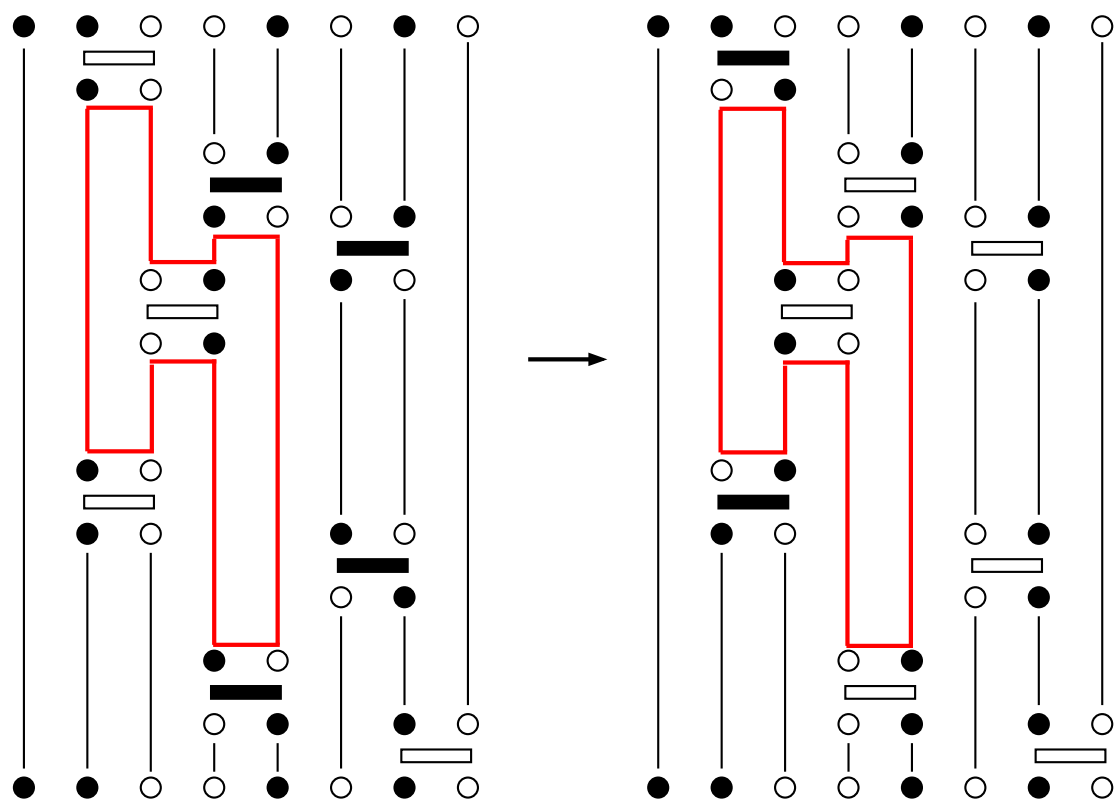

Figura 7.4: Configuração da amostra de Monte Carlo com um loop destacado mostrando a orientação dos spins antes e depois da operação do inversão dos spins. Todos os spins situados nos vértices do loop são invertidos, assim os operadores tem seus tipos alterados $[1, b]_{p} \leftrightarrow[2, b]_{p}$. Operadores tocados duas vezes pelo loop mantém seu tipo enquanto a orientação de todos os spins que o cercam são invertidas. Cada loop é unicamente determinado e cada spin pertence a apenas um loop.

todas as orientações de spins invertidas. Repare que implicitamente altera-se a orientação de todos os spins que se encontram no caminho entre os operadores.

Os loops são construídos de tal modo que cada spin pertença a apenas um loop. Um dado loop segue o caminho de spins constantes, e muda de direção quando encontra um operador. Dessa forma, os loops são definidos de maneira única.

Além de ser uma maneira muito eficiente para atualizar os operadores, outro aspecto fundamental, é que essas atualizações sempre podem ser aceitas, pois o peso de cada configuração depende apenas do número de operadores, e não do tipo de operadores, veja equação 7.17. Como não altera o número de operadores, a atualização de um loop está sempre de acordo com as regras do balanceamento detalhado.

Na prática, a melhor estratégia é construir todos os loops possíveis de uma certa configuração, e inverter a orientação de todos os spins que pertencem a cada loop com probabilidade 1/2. Essa regra em conjunto com a regra para atualização dos operadores 
diagonais garante que a amostra de Monte Carlo seja ergódica, ou seja, que toda configuração possível pode ser alcançada eventualmente. Vale a pena salientar que a introdução dos operadores diagonais discutida anteriormente constitui um mecanismo pelo qual os loops são alterados.

Uma boa definição do passo de Monte Carlo consiste de uma sequência completa de atualizações de operadores diagonais $(p=1, \ldots, L)$, seguida da construção de todos os loops, e inversão desses loops com probabilidade $1 / 2$.

O operador loop que acabamos de apresentar é restrito ao modelo de Heisenberg de spin $1 / 2$. Porém, esse conceito pode ser generalizado para uma gama muito maior de modelos, incluindo o modelo XXZ, modelos com spins de magnitude maior, com campo externo, além de modelos com bósons. A generalização chamada de directed loop update [47] consiste na construção de loops levando em conta configurações mais diversificadas de operadores de par e spins, chamados de vértices, que leva em conta a diversidade desses modelos, além de ser mais eficiente.

\section{Médias de observáveis.}

Por fim comentamos brevemente como médias de observáveis podem ser calculadas a partir da amostragem do Monte Carlo que acabamos de construir. Na equação (7.7) já havíamos mostrado como estimar a energia do sistema. O calor específico pode ser calculado de maneira similar tendo como resultado

$$
C(T)=\left\langle n^{2}\right\rangle-\langle n\rangle^{2}-\langle n\rangle
$$

Em geral operadores diagonais nos estados da base $S^{z}$ são facilmente estimados como

$$
\bar{O}_{z}=\frac{1}{L} \sum_{p=0}^{L-1}\left\langle\alpha(p)\left|O_{z}\right| \alpha(p)\right\rangle=\frac{1}{L} \sum_{p=0}^{L-1} O_{z}(p),
$$

onde não é necessário que se calcule o valor do operador para todos os valores de $p$, podendo-se simplificar o cálculo como uma soma parcial sobre $p, O_{z}(0)+O_{z}(P)+O_{z}(2 P)+$ $\cdots$, para obter uma medida mais eficiente, já que os estados vizinhos em $p$ diferem entre si no máximo pela inversão de dois spins. 
Com isso pode-se calcular facilmente a susceptibilidade magnética a campo nulo como

$$
\chi(T)=\frac{\beta}{N}\left\langle M_{z}^{2}\right\rangle, \quad \text { onde } \quad M_{z}(p)=\sum_{i=1}^{N} S_{i}^{z}(p) .
$$

O erro associado às medidas no Monte Carlo quântico surge devido à quantidade finita de configurações alcançadas nas atualizações da amostra. Como dito anteriormente, a truncagem da soma na função de partição não causa nenhum erro mensurável. Podemos estimar o erro dividindo o tempo de simulação, passos de Monte Carlo, em $B$ intervalos, contendo $N$ passos cada intervalo. Para um observável $Q$, fazemos a média em cada intervalo $\bar{Q}_{b}(\operatorname{com} b=1,2, \ldots, B)$, para então estimar a média e o desvio padrão da média do observável como

$$
\bar{Q}=\frac{1}{B} \sum_{b=1}^{B} \bar{Q}_{b} ; \quad \text { onde } \quad \sigma_{Q}=\frac{1}{B(B-1)} \sum_{b=1}^{B}\left(\bar{Q}_{b}-\bar{Q}\right)^{2}
$$

Se $N$ é muito maior do que o tempo de correlação da grandeza medida, as médias nos intervalos $B$ podem ser consideradas independentes, portanto, podemos calcular o erro (desvio padrão) na forma escrita acima. Assim, a média do observável $Q$ é dada por

$$
\langle Q\rangle=\bar{Q} \pm \sigma_{Q}
$$

Na prática não é necessário conhecer o tempo de correlação da grandeza medida, pois é possível calcular as médias para valores variáveis de $N$, por exemplo, começando de 100 até 1000, checando quando o desvio padrão converge. Isso garante que $N$ seja maior que o tempo de correlação.

Retornamos agora ao estudo das cadeias quânticas de Heisenberg aperiódicas. O objetivo é comparar os resultados analíticos obtidos pelo SDRG com resultados numéricos do QMC. 


\subsection{Simulações de QMC para a cadeia de Fibonacci- Heisenberg.}

No regime de modulação forte $(r \gg 1)$, a cadeia de Fibonacci-Heisenberg pode ser aproximada como um conjunto de pares independentes, acoplados em estados de singletos pela ligação efetiva $J_{b}$. No caso da cadeia de spin $1 / 2$, para a qual o estado fundamental deve ser uma fase de singleto aperiódica [34], esta aproximação deve ser qualitativamente correta para todas as temperaturas, desde de que a modulação seja forte o suficiente. Por outro lado, no caso de spins 1, esse aproximação deve ser válida somente até temperaturas acima do gap $\Delta(r)$ da equação (5.6). Portanto, podemos estimar a energia livre da cadeia de Fibonacci-Heisenberg como

$$
f(B, T)=\frac{1}{2} \sum_{j=0}^{j^{*}}\left(n_{j}-n_{j+1}\right) F_{\text {pair }}\left(J_{b}^{(j)} ; B, T\right)
$$

onde $F_{\text {pair }}\left(J_{b}^{(j)} ; B, T\right)$ é a energia livre de uma par de spins interagindo pela hamiltoniana

$$
H_{\text {pair }}=J_{b}^{(j)} \mathbf{S}_{1} \cdot \mathbf{S}_{2}-B\left(S_{1}^{z}+S_{2}^{z}\right)
$$

(com o campo magnético fraco $B$, introduzido para que possamos calcular a susceptibilidade magnética), $n_{j}$ é a fração de spins ativos (ainda não dizimados) na $j$-ésima iteração do SDRG, e $j^{*}$ é a iteração na qual a razão efetiva entre os acoplamentos se torna da ordem de 1. A partir da discussão acima é claro que $j^{*}=\infty$ para cadeias de spin $1 / 2$, enquanto para a cadeia de spin $1 j^{*}$ é dado por

$$
j^{*}=\left[\frac{\ln r}{\ln 4 / 3}\right]_{\text {inteiro }} .
$$

Para as iterações anteriores a $j^{*}$, como os acoplamentos efetivos sempre seguem a sequência de Fibonacci, a fração de spins ativos satisfaz a equação de recorrência $n_{j+1}=$ $\left(1-2 f_{b}\right) n_{j}$, onde $f_{b}=(3-\sqrt{5}) / 2 \simeq 0.382$ é a fração de letras $b$ em uma sequência de Fibonacci infinita (veja a seção 2). 


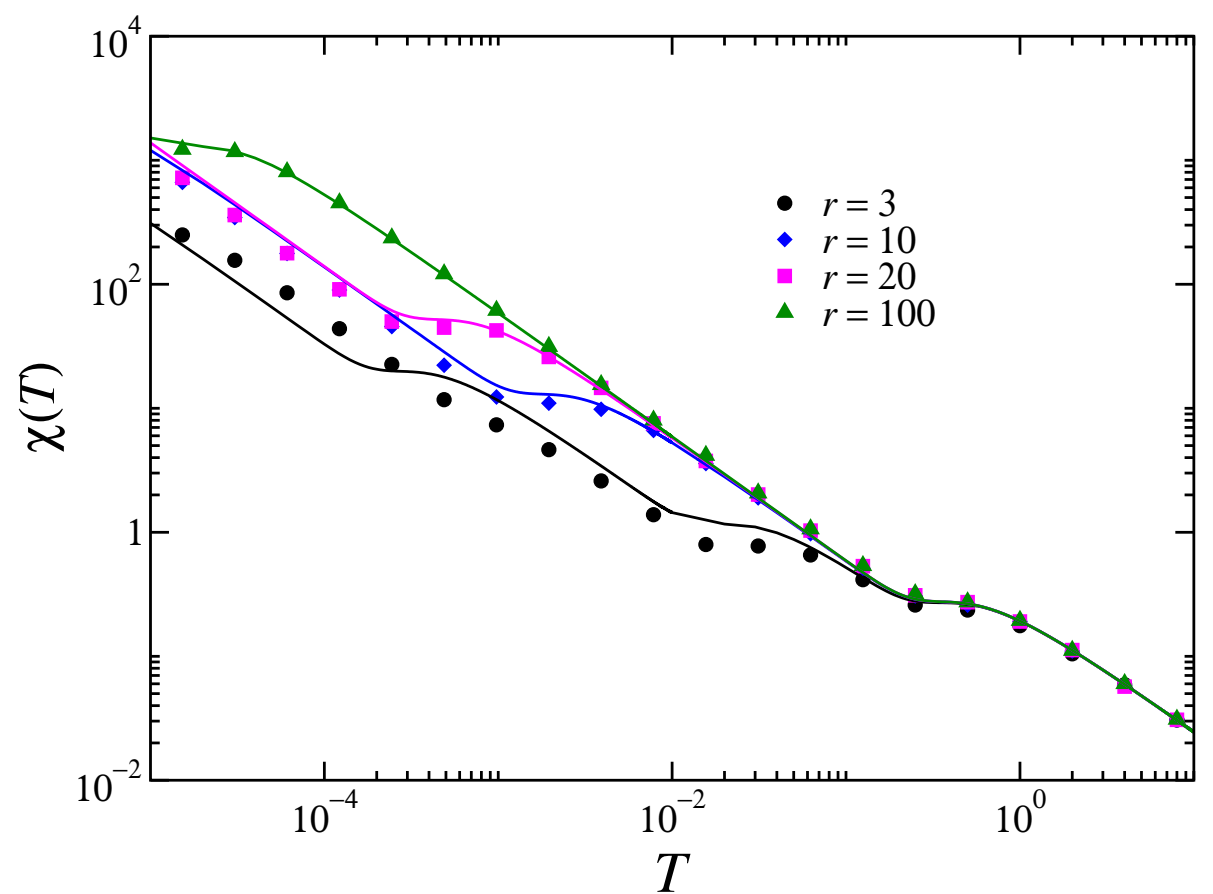

Figura 7.5: Susceptibilidade magnética como função da temperatura para a cadeia de Fibonacci-Heisenberg de spin 1/2. Linhas sólidas correspondem à previsão do SDRG para diferentes valores da razão entre acoplamentos $r=J_{b} / J_{a}$. O resultados de QMC (símbolos) foram obtidos usando cadeias com 90 sítios e condições de contorno abertas. Barras de erro são menores que os símbolos. Simulação feita pelo colaborador Nicolas Laflorencie.

A susceptibilidade a campo nulo é então obtida pela energia livre como

$$
\chi(T)=-\left.\frac{\partial^{2} f}{\partial B^{2}}\right|_{B=0} .
$$

Primeiro checamos as previsões do SDRG para a cadeia de spin 1/2, usando os acoplamentos efetivos calculados na referência [34], e comparando os resultados das equações (7.27) e (7.30) com simulações de QMC, usando o método de expansão em séries estocásticas $S S E$ [46, 48], com directed loop update [47]. Como mostra a figura 7.5, a previsão de SDRG fica mais próxima dos resultados de QMC conforme a modulação cresce, como esperado pela natureza perturbativa do SDRG.

Para a correspondente cadeia de spin 1, figuras 7.6 e 7.7 mostram a susceptibilidade como função da temperatura de acordo com a segunda e terceira abordagens, juntamente 


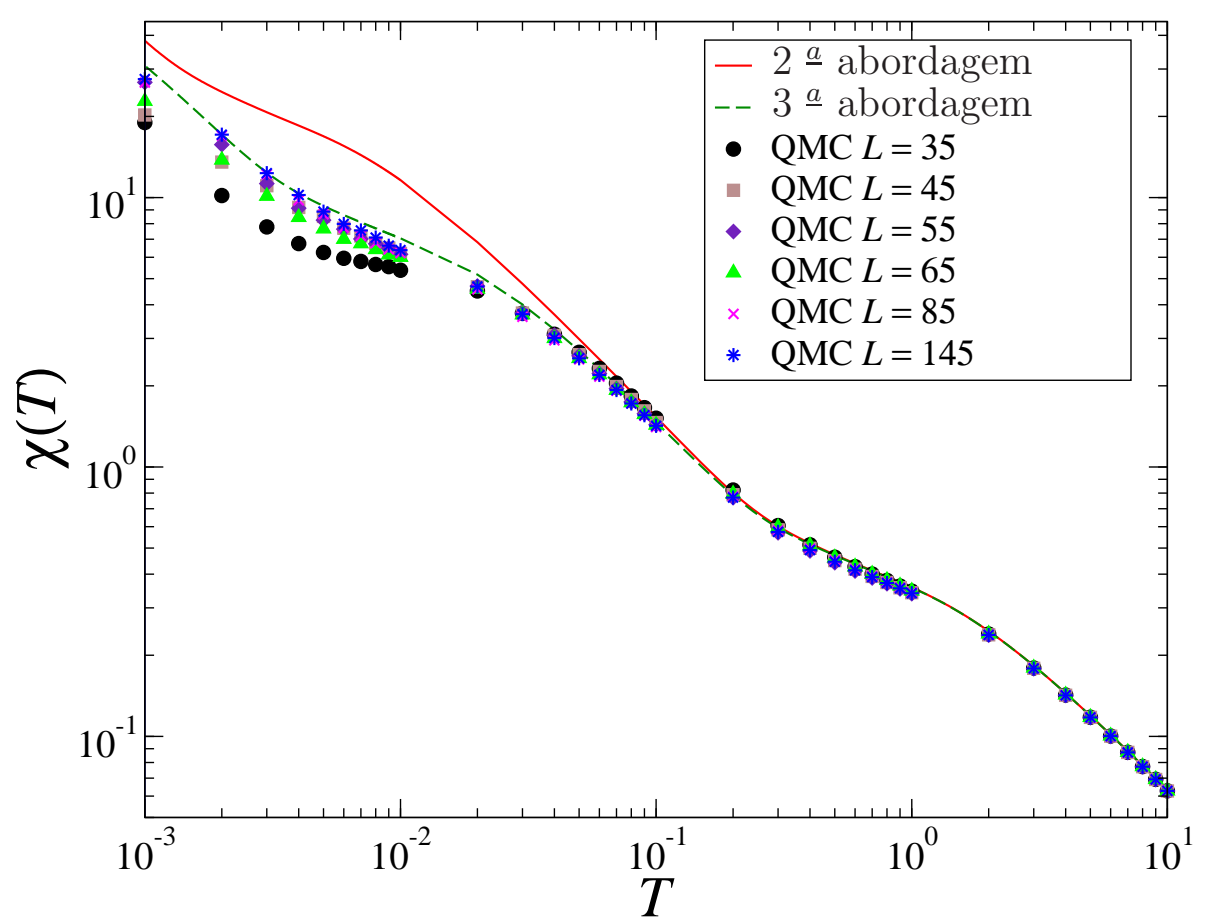

Figura 7.6: Susceptibilidade magnética em função da temperatura para a cadeia de Fibonacci-Heisenberg de spin 1 com razão $r=10$. As linhas sólidas (tracejadas) correspondem às previsões da segunda (terceira) abordagem, enquanto os símbolos correspondem a resultados de QMC para diferentes tamanhos da cadeia $L$. Barras de erro são menores que os símbolos.

com dados de QMC, para a razão entre acoplamentos $r=10$ e $r=20$, respectivamente. Claramente a concordância com os dados de baixa temperatura é significantemente melhor para a terceira abordagem, e se torna ainda melhor conforme o número de spins $L$ aumenta. Repare nos "ombros" que a curva da susceptibilidade apresenta nas proximidades de temperaturas relacionadas a escalas de energia das ligações efetivas $J_{b}$.

Repare também que as curvas de QMC se aproximam da curva calculada pelo SDRG (terceira abordagem) de forma não-monotônica conforme aumentamos o tamanho da cadeia. Para entender isso, precisamos lembrar que a fração de letras $b$ em uma sequência finita sofre um desvio, em relação a fração de letras $b$ em uma sequência infinita, esse desvio alterna valores positivos e negativos à medida que as iteradas da regra de substituição se sucedem. Assim, podemos entender a não monotonicidade como o efeito da flutuação da fração de letras $b$, ou seja, da flutuação do número de singletos fortemente 


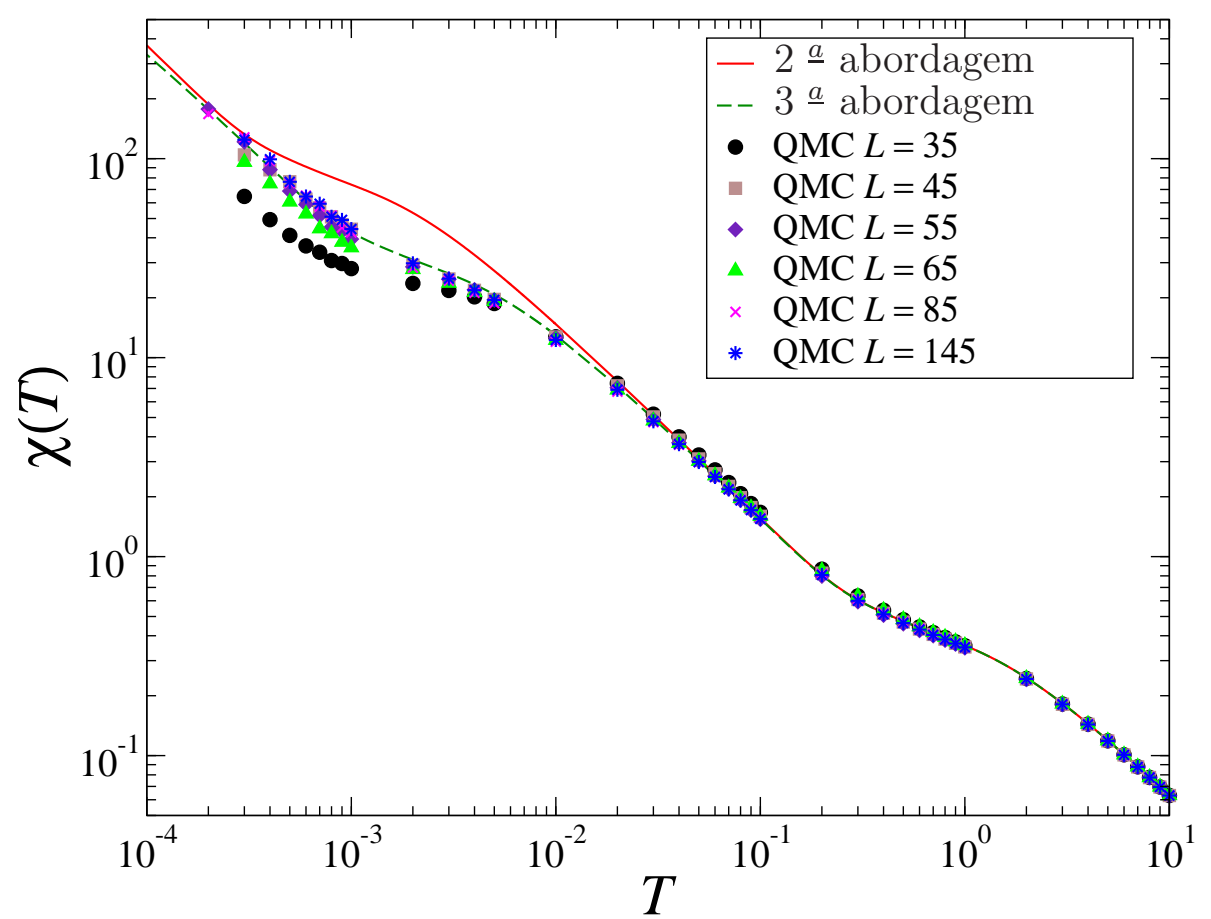

Figura 7.7: Susceptibilidade magnética em função da temperatura para a cadeia de Fibonacci-Heisenberg de spin 1, similar a figura 7.6, porém com a razão entre acoplamentos $r=20$.

acoplados em cadeias de tamanho finito.

Utilizamos nos cálculos de QMC cadeias com número ímpar de spins. Assim a susceptibilidade não se anula em baixas temperaturas mesmo quando o estado fundamental tem um gap, pois há sempre um spin livre desacoplado, ou seja, que não faz parte de um singleto. Se utilizarmos cadeias com um número par de spins, o gap da cadeia finita, que é maior do que o gap de uma cadeia infinita previsto na equação (5.6) $\left(\Delta \sim 10^{-8} J_{b}\right)$, faz a susceptibilidade magnética se anular para temperaturas da ordem do gap. Nos casos estudados nas figuras 7.6 e 7.7. o gap é da ordem dos valores de temperaturas estudados, como pode ser visto na figura 8.1 . 


\subsection{Simulações de QMC para a cadeia de Heisenberg- 6-3.}

Usando a mesma aproximação de singletos independentes descrita para a cadeia de Fibonacci-Heisenberg na seção [7.2, podemos obter, a partir das previsões do SDRG, a susceptibilidade a campo zero quando os acoplamentos seguem a sequência 6-3. Algumas pequenas adaptações são necessárias, pois a distribuição autossimilar de acoplamentos é diferente da sequência 6-3. Assim, devemos levar em conta que a fração de ligações $J_{b}$ na cadeia original é $f_{b}=\frac{1}{3}$, enquanto a fração de ligações $J_{1}$ na distribuição autossimilar é dada por $f_{J_{1}}=\frac{2}{5}$. Também temos que $j^{*}=\infty$. Logo abaixo, os resultados com a aproximação de singletos independentes são comparados com dados de QMC.

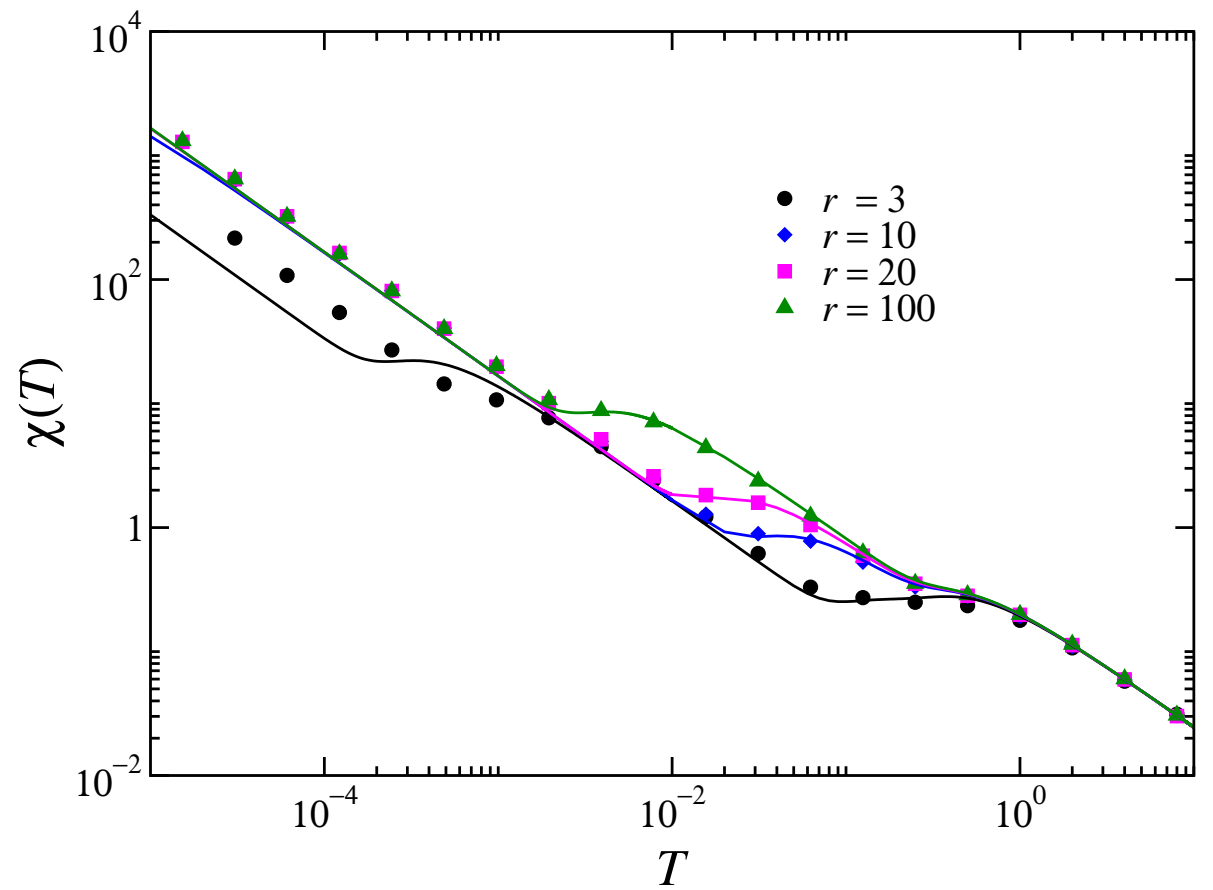

Figura 7.8: Susceptibilidade magnética em função da temperatura para a cadeia de Heisenberg-6-3 de spin 1/2. Linhas sólidas são as previsões do SDRG para várias razões de acoplamentos $r=J_{b} / J_{a}$, enquanto símbolos indicam os correspondentes resultados de QMC com $L=75$ sítios. Simulação feita pelo colaborador Nicolas Laflorencie.

Para a cadeia de spins $1 / 2$ com $L=75$ sítios os resultados são mostrados na figura 7.8. Como esperado, a concordância entre as previsões do SDRG e as simulações de QMC 
é melhor para uma razão entre os acoplamentos grande $r=J_{b} / J_{a}$.

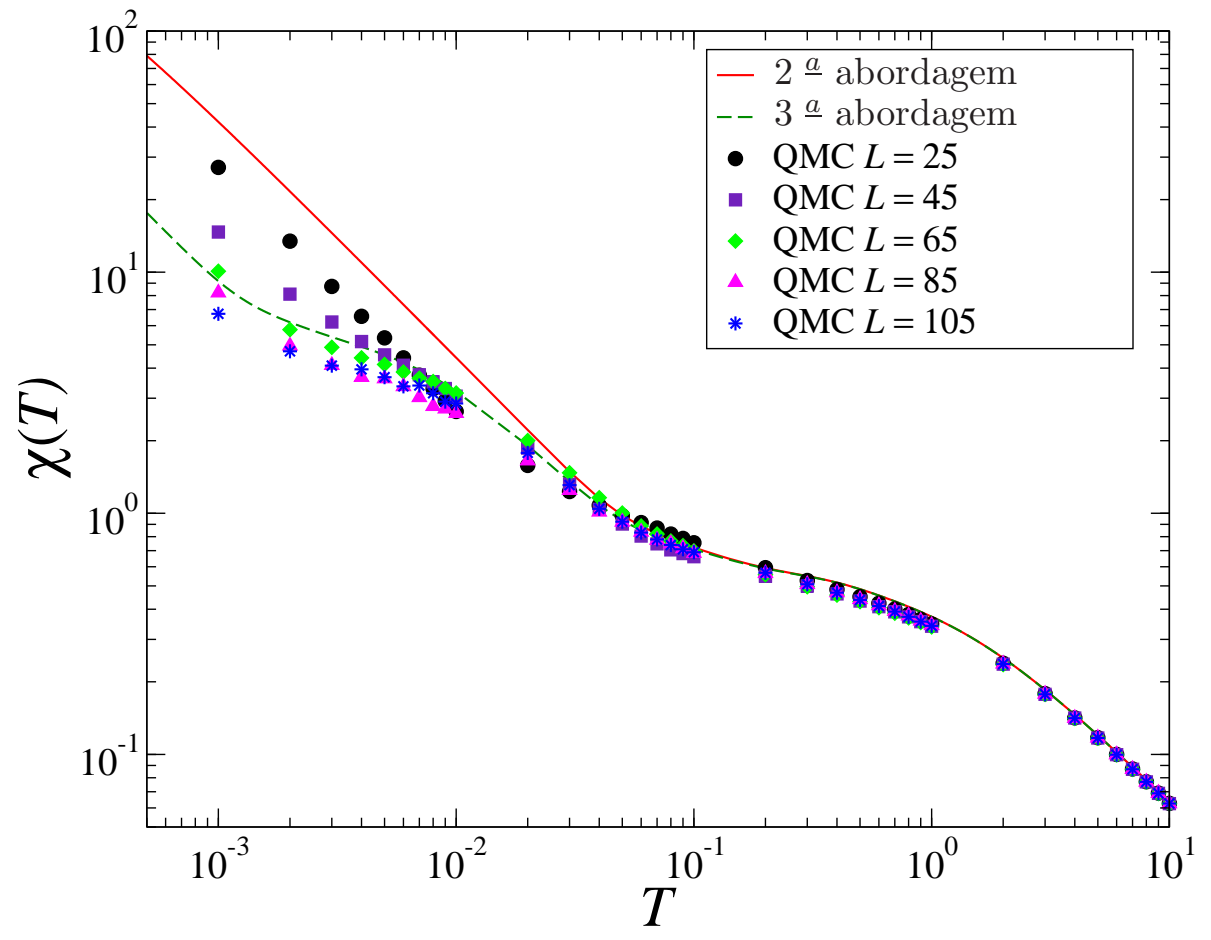

Figura 7.9: Susceptibilidade magnética em função da temperatura para a cadeia de Heisenberg-6-3 de spin 1 com razão $r=5$. Linhas sólidas (tracejadas) correspondem a previsões do SDRG de acordo com a segunda (terceira) abordagem, enquanto símbolos correspondem a resultados de QMC para diferentes tamanhos de cadeia $L$. Barras de erro são menores que os símbolos.

Nas figuras 7.9 e 7.10 mostramos os resultados para a cadeia de spin 1 com a razão entre os acoplamentos $r=5$ e $r=10$ respectivamente. Assim como no caso da cadeia de Fibonacci-Heisenberg, é claro que os resultados de QMC mostram uma melhor concordância com as previsões da terceira abordagem do SDRG.

Repare que novamente as curvas de QMC não se aproximam da curva calculada pelo SDRG (terceira abordagem) de forma monotônica conforme aumentamos o tamanho da cadeia, devido as flutuação dos números de letras b em uma sequência finita. Novamente escolhemos sempre cadeias com um número ímpar de spins. 


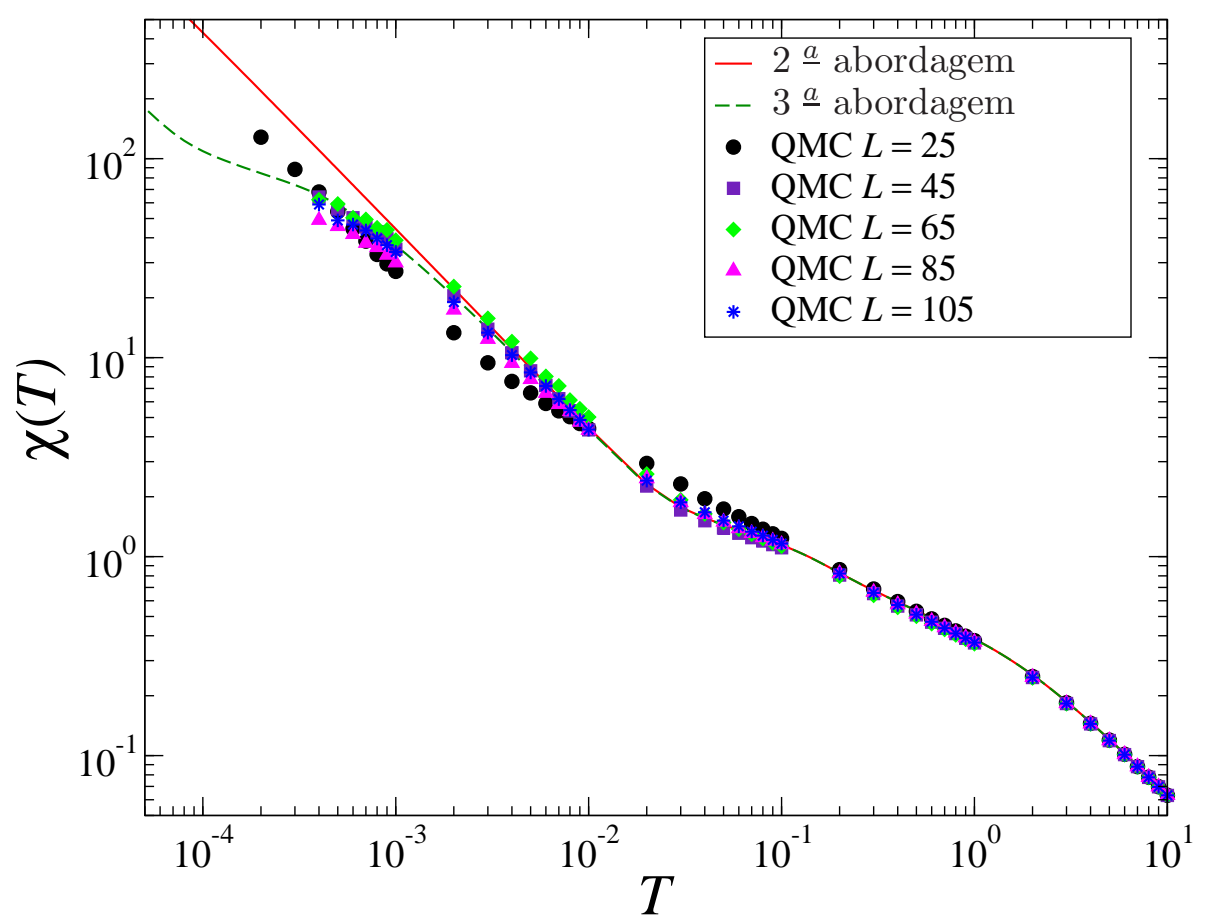

Figura 7.10: Susceptibilidade magnética em função da temperatura para a cadeia de Heisenberg-6-3 de spin 1, similar a figura 7.9, porém com razão entre acoplamentos $r=10$. 


\section{Capítulo 8}

\section{Grupo de renormalização da matriz densidade.}

Para verificar se uma razão suficientemente grande entre os acoplamentos $J_{a}$ e $J_{b}$ pode ou não induzir uma transição de fase no sistema para uma fase sem gap, dominada pela aperiodicidade, apresentamos estudos numéricos utilizando o método de DMRG para calcular o gap e para o parâmetro de ordem de corda na cadeia de Fibonacci-Heisenberg, para diversos valores da razão entre os acoplamentos. Os resultados apresentados neste capítulo foram obtidos por meio de uma colaboração com os pesquisadores Fabien Alet e Nicolas Laflorencie da Université Paul Sabatier de Toulouse - França. Essa colaboração científica resultou na publicação do artigo [35], de onde reproduzimos as duas seções seguintes a partir dos resultados de DMRG desse trabalho.

O grupo de renormalização da matriz densidade é um método numérico muito eficiente no estudo de propriedades de baixas temperaturas de sistemas quânticos fortemente correlacionados. O método baseia-se na construção dos estados mais relevantes em baixas temperatura, truncando-se o espaço de Hilbert para eliminar os demais estados. Diferentemente do que ocorre com o SDRG onde iniciamos com uma cadeia de spins, e iterativamente os spins associados a graus de liberdades importantes em altas temperaturas são eliminados e ligações efetivas são geradas, no DMRG, inicia-se com um sistema pequeno, que é então desenvolvido pelo acréscimos de novos spins para compor a cadeia. Em cada iteração o hamiltoniano é diagonalizado, e apenas os estados mais significantes são preservados. 
O método é construído com a iteração do seguinte procedimento. Inicialmente tem se um bloco $B$ contendo um spin, com hamiltoniano $\mathcal{H}_{B}$ que contém os termos do hamiltoniano em questão relacionados a este spin. Constrói-se um super bloco que contém a direita e a esquerda blocos $B$, entre ambos há dois spins. A interação entre esses componentes do superbloco é dado pelo hamiltoniano do sistema. Após a diagonalização do hamiltoniano do superbloco, constrói-se a matriz densidade do superbloco. Os maiores autovalores da matriz densidade são os autovalores que devem ser preservados. Com os estados mais significativos se constrói um novo bloco $B^{\prime}$ formado pelo bloco $B$ e por um dos spins centrais. Novos operadores que compõe o hamiltoniano são construídos no subespaço desses estados. Assim tem-se um novo hamiltoniano para o bloco $B^{\prime}$. Por fim faz-se $B=B^{\prime}$ e repete-se o procedimento descrito. Para leitores interessados em detalhes aprofundados do método de DMRG recomendamos a referência [10].

\subsection{Cálculo do gap e do parâmetro de ordem de corda na cadeia de Fibonacci-Heisenberg de spin 1.}

Foi simulada uma cadeia aperiódica com $L$ de spins 1, com condições de contorno abertas, usando o método de DMRG [10] com o formalismo de product state [49. Foi usada a formulação da simetria de $\mathrm{SU}(2)$ [50], aproveitando a simetria da hamiltoniana (1.1), o que reduz consideravelmente o número de estados $m$, para serem armazenados nos cálculos de DMRG. A convergência do estado fundamental em diferentes setores $S_{T}=$ 0,1 ou 2 é particularmente difícil de alcançar para um $L$ grande e uma razão $r$ forte, o que atribuímos à aperiodicidade do sistema. Para garantir a convergência, usou-se procedimentos específicos de aquecimento onde aumenta-se sequencialmente o número de estados $m$ de $\mathrm{SU}(2)$ armazenados, tipicamente 20 ou 50, até valores de $m$ onde a energia do estado fundamental não varie mais. Para a maior cadeia de Fibonacci (aqui $L=378$ ), o número máximo de estados $\mathrm{SU}(2)$ foi de $m=700$, correspondendo a aproximadamente 3600 estados U(1). Para cada valor de $m$ no procedimento de aquecimento, executou-se um grande número de varreduras (algumas vezes mais que 200), novamente verificando se a energia não varia.

Dependendo da paridade no número de spins da cadeia, tamanho $L$, o estado fundamental foi encontrado no setor $S_{T}=0$ (para $L$ par) ou no setor $S_{T}=1$ ( $L$ ímpar), como 


\subsection{Cálculo do gap e do parâmetro de ordem de corda na cadeia de Fibonacci-Heisenberg de spin 1.}

esperado. Na fase de Haldane, espera-se que a diferença entre a energia dos dois setores decaia exponencialmente com o aumento de $L$ em cadeias abertas, devido à presença de graus de liberdade de spin $1 / 2$ nas bordas 1 De forma similar ao que foi feito no estudo original da cadeia uniforme de spin 1 [10], foi calculado o gap $\Delta$ como a diferença entre este estado fundamental e a energia do estado fundamental no setor $S_{T}=2$, ou seja, $\Delta=E_{0}\left(S_{T}=2\right)-E_{0}\left(S_{T}=0 / 1\right)$.

Foram simuladas cadeias com tamanhos $L=22,35,56,90,145,234,378$ que correspondem aos comprimentos naturais (da sequência de Fibonacci) de $L-1=21,34,55,89,144$, 233, 377. São apresentados a seguir resultados somente para comprimentos naturais de Fibonacci, porém, para cadeias com um comprimento qualquer $L<70$, foi verificado que o comportamento é qualitativamente o mesmo quando se faz uma média sobre todas as $L+1$ possíveis subsequências de Fibonacci, que podem ser encontradas em uma sequência finita de $L$ letras.

A figura 8.1 mostra os resultados para o gap $\Delta$ (em unidades de $J_{a}$ ), como função da razão $r$, para diferentes tamanhos $L$. A partir da figura é claro que o gap não se anula no intervalo $r \in[1,6]$ simulado, ainda assim, como esperado, o gap decresce consideravelmente com o aumento de $r$. Também podemos observar efeitos da não monoticidade devido a flutuação do números de letras em cadeias finitas.

Note que o gap calculado via DMRG não é diretamente comparável com os preditos pela equação (5.6), que é válida somente no limite de uma cadeia infinita e modulação forte, desconsiderando assim efeitos de borda. Efeitos de borda se tornam muito importantes para cadeias pequenas, aquelas acessíveis via DMRG. No gráfico inserido na figura 8.1 mostra-se a comparação entre os dados de DMRG para o gap, com modulação mais forte $(r=6)$ para a qual os dados estão disponíveis, e as correspondentes previsões de SDRG para cadeias abertas de tamanho finito. Detalhes de como pode-se calcular o gap para cadeias finitas via SDRG são dados no apêndice C. A concordância é melhor para cadeias pequenas, discrepâncias surgem quando $L \geq 56$, devido ao fato de que a razão de acoplamentos efetivos decresce iterativamente, equação (5.3). Assim com o aumento da cadeia há um número maior de spins para serem dizimados e os cálculos perturbativos sobre os quais o SDRG é construído tornam-se menos precisos, levando a erros na estimativa do gap. Mesmo assim, para cadeias grandes ( $L=234$ e $L=378)$, as curvas

\footnotetext{
${ }^{1}$ Também foi realizado cálculos para condições periódicas de contorno, porém a convergência não foi satisfatória.
} 


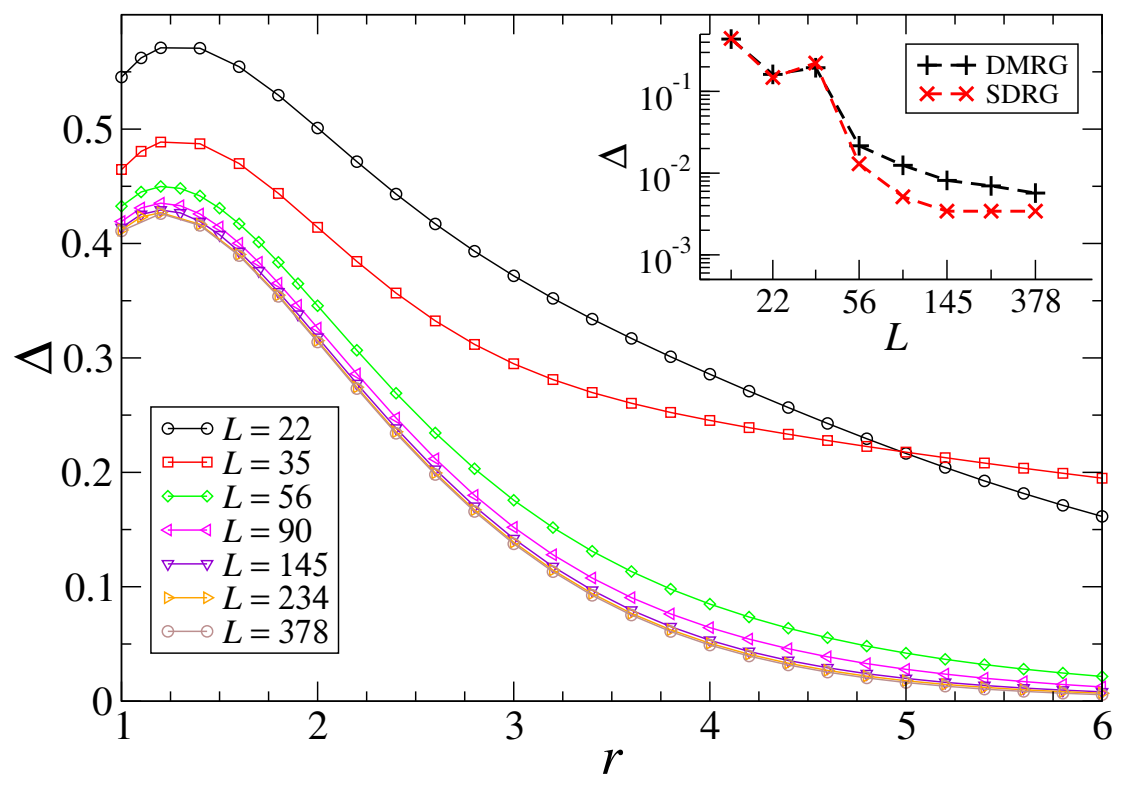

Figura 8.1: Cadeia de spin 1 modulada pela sequência de Fibonacci. Gap $\Delta=E_{0}\left(S_{T}=\right.$ $2)-E_{0}\left(S_{T}=0 / 1\right)$ entre o nível mais baixo de energia de quintupleto $E_{0}\left(S_{T}=2\right)$ e o estado fundamental $E_{0}$ (o qual é um estado de singleto $S_{T}=0$ ou um estado de tripleto $S_{T}=1$, dependendo da paridade da cadeia), como função da razão entre os acoplamentos $r=J_{b} / J_{a}$, para diferentes tamanhos $L$. Esta simulação foi efetuada pelo colaborador Fabien Alet.

claramente se aproximam.

\section{Parâmetro de ordem de corda.}

Os resultados prévios indicam que a fase de Haldane não é destruída pela modulação de Fibonacci nos acoplamentos da cadeia de Heisenberg. Pode-se confirmar este resultado pelo cálculo via DMRG da função de correlação de corda [8]

$$
\left\langle O^{z}(i, j)\right\rangle=\left\langle S_{i}^{z} \exp \left(\mathrm{i} \pi \sum_{k=i+1}^{j-1} S_{k}^{z}\right) S_{j}^{z}\right\rangle,
$$

como função da distância $x=|j-i|$. A função correlação de corda assume valores não nulos, no limite de longas distâncias, na fase de Haldane [8], sendo assim um bom indicador da continuidade da fase de Haldane quando a razão entre os acoplamentos aumenta.

É apresentado na figura $8.2\left\langle O^{z}(x=|j-i|)\right\rangle$ com $i=L / 4$ e $x$ variando de 0 a $L / 2$ 
8.2 Cálculo do gap e do parâmetro de ordem de corda na cadeia de Heisenberg-6-3 de spin 1.

(esse intervalo minimiza os efeitos das condições de contorno abertas). Foram escolhidos alguns valores de $r$, e o maior sistema simulado $L=378$. Uma função de correlação no espaço real como $\left\langle O^{z}(x)\right\rangle$ é inevitavelmente não monotônica para um sistema aperiódico, porém os resultados da figura 8.2 indicam claramente que o parâmetro de ordem de corda não se anula até o valor de $r=6$, mesmo que este alcance um valor menor (em $x \rightarrow \infty)$ quando $r$ cresce, como esperado do comportamento do gap.

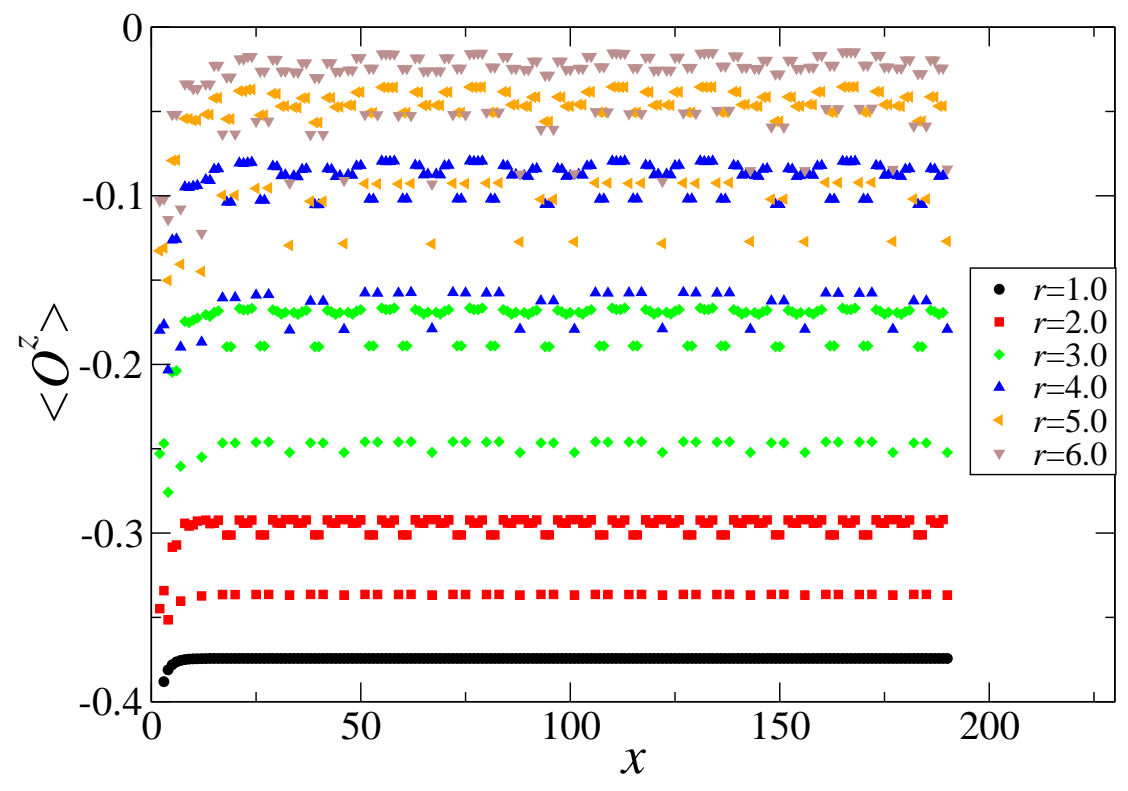

Figura 8.2: Cadeia de spin 1 modulada pela sequência de Fibonacci: função de correlação de corda $\left\langle O^{z}(x=|i-j|)\right\rangle$ como uma função da distância $x$. O ponto de partida é $i=L / 4$ até o valor máximo de $x=L / 2$, para uma cadeia de $L=376$ e diferentes valores da razão $r=J_{b} / J_{a}$. Esta simulação foi efetuada pelo colaborador Fabien Alet.

Em geral, os resultados de DMRG para o gap e para o parâmetro de ordem de corda estão de acordo com as conclusões do SDRG (abordagens 1 e 3) de que a fase de Haldane com gap sobrevive à introdução da aperiodicidade de Fibonacci.

\subsection{Cálculo do gap e do parâmetro de ordem de corda na cadeia de Heisenberg-6-3 de spin 1.}

Foi usado aqui o mesmo procedimento descrito na seção anterior para calcular o gap $\Delta$ (em unidades de $J_{a}$ ) em cadeias de spin 1 moduladas pela sequência 6-3. Foram escol- 
hidos os tamanhos $L=45,75,85,105,325,376$ e mostram-se os resultados na figura 8.3, Esses resultados mostram claramente que o gap se anula para um sistema suficientemente grande, quando a razão $r$ também é forte o suficiente. Para os maiores sistemas consider$\operatorname{ados}(L=325$ e $L=376)$, as simulações não convergem para valores maiores que $r \geq 4$, porém o comportamento para menores valores de $r$ e $L$ claramente indica que o gap se anula para esses casos.

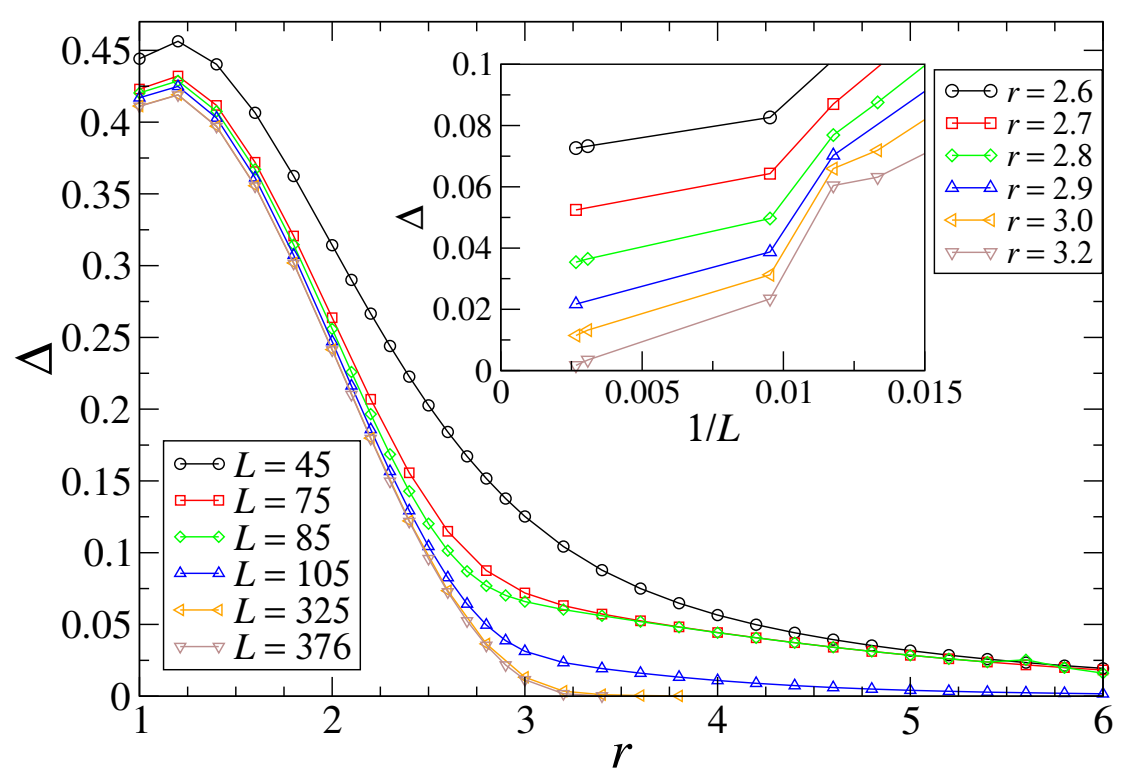

Figura 8.3: Cadeia de spin 1 modulada pela sequência 6-3: gap $\Delta=E_{0}\left(S_{T}=2\right)-E_{0}\left(S_{T}=\right.$ $0 / 1)$ entre a energia do estado de quintupleto $S_{T}=2$ de menor energia $E_{0}\left(S_{T}=2\right)$ e o estado fundamental $E_{0}$ (o qual é um singleto $S_{T}=0$ ou um tripleto $S_{T}=1$, dependendo da paridade da cadeia), como função da razão entre os acoplamentos $r=J_{b} / J_{a}$, para diferentes tamanhos $L$ do sistema. Esta simulação foi efetuada pelo colaborador Fabien Alet.

Esses resultados novamente estão em acordo com os cálculos de SDRG, os quais indicam que o gap de Haldane deve se anular acima de uma modulação crítica $r_{c}$, dando origem a uma fase de singleto aperiódico sem gap.

O valor crítico $r_{c}$ no qual esta transição de fase quântica acontece é difícil de ser estabelecido devido aos fortes efeitos de tamanho finito que surgem quando o gap é muito pequeno. Mesmo assim, considerando o maior sistema disponível, podemos estimar que o gap é nulo para $r=3.4$. O gráfico inserido na figura 8.3 mostra o gap como função do inverso do tamanho do sistema $1 / L$, para valores da razão entre os acoplamentos próximos 


\subsection{Cálculo do gap e do parâmetro de ordem de corda na cadeia de}

Heisenberg-6-3 de spin 1.

da transição. Poderia-se estimar o valor crítico $r_{c} \approx 2.9(2)$, porém essa determinação fenomenológica deve ser feita com cuidado.

A previsão do SDRG $r_{c} \simeq 1.73$ (da terceira abordagem) é diferente, e está sujeita a grandes incertezas, devido à natureza imprecisa dos cálculos de teoria de perturbação nessa região de parâmetros. Ainda assim podemos concluir que os cálculos numéricos do gap confirmam a previsão do SDRG de uma fase sem gap dominada pela aperiodicidade quando a razão $r$ é forte o suficiente, porém finita.

Finalmente pode-se confirmar essa previsão calculando a função de correlação de corda $\left\langle O^{z}(x=|j-i|)\right\rangle$ usando-se o mesmo método da seção 8.1. Na figura 8.4 são apresentados os resultados das simulações para a cadeia com maior comprimento $L=376$ para a qual atingiu-se a convergência para $r=1,2,3$ (figura superior) e para uma cadeia menor $L=105$ onde atingiu-se a convergência suficiente para valores até $r=6$ (figura inferior), para valores inteiros de $r$.

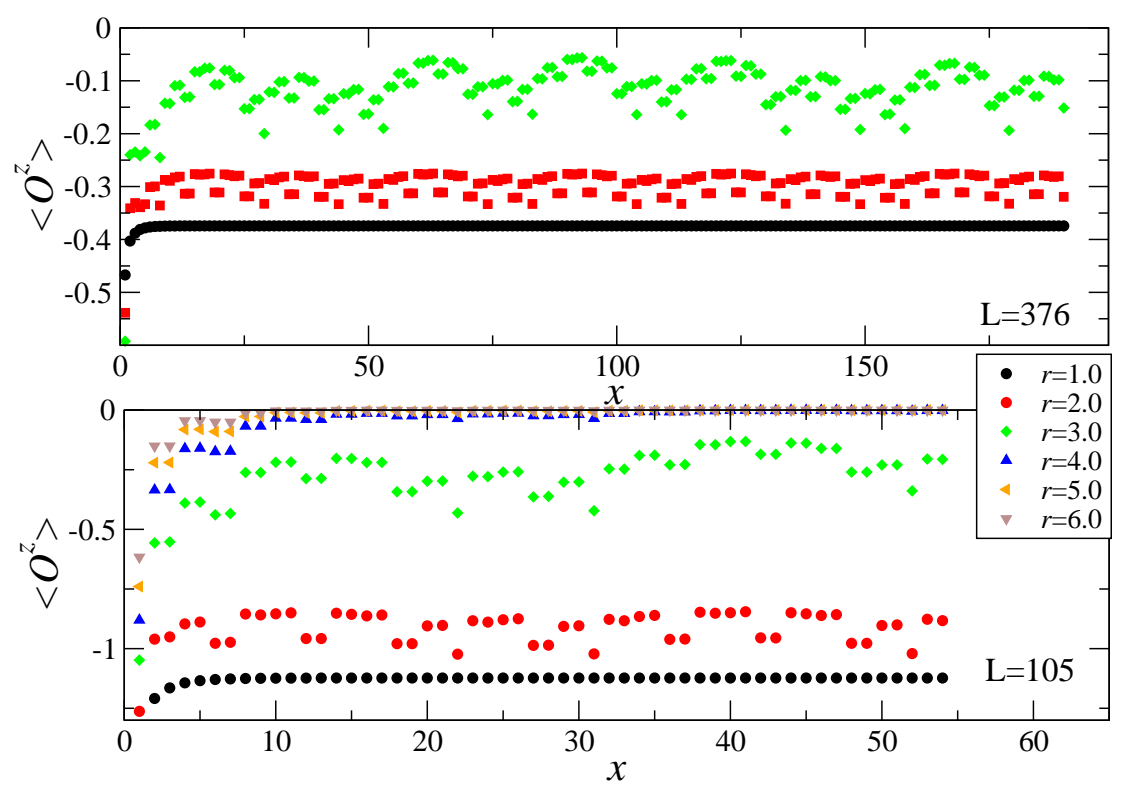

Figura 8.4: Cadeia de spin 1 modulada pela sequência 6-3: função de correlação de corda $\left\langle O^{z}(x=|i-j|)\right\rangle$ como função da distância $x$. O ponto de partida é $i=L / 4$ até o valor máximo de $x=L / 2$, para uma cadeia de $L=376$ (figura superior) e para uma cadeia de $L=105$ (figura inferior) para diferentes valores da razão $r=J_{b} / J_{a}$. Esta simulação foi efetuada pelo colaborador Fabien Alet.

Para $L=376$, o comportamento de longa distância de $\left\langle O^{z}(x)\right\rangle$ indica que a fase de Haldane ainda está presente na amostra de tamanho finito até o valor de $r=3$, porém 
com um valor menor para o parâmetro de ordem do que valores menores de $r$, de acordo com o menor gap encontrado para esse sistema. Por outro lado, para $r=4,5,6$, os resultados para um sistema menor $L=105$ claramente indicam que o parâmetro de ordem de corda se anula no limite de longa distância, confirmando assim que a fase de Haldane foi destruída. Devido ao comportamento não regular em $x$, não foi possível fazer uma análise de escala finita no parâmetro de ordem para diferentes tamanhos do sistema e estimar o valor crítico de $r_{c}$, porém, nossos resultados para o maior sistema $L=376$ são consistentes com nossa estimativa $r_{c}=2.9(2)$ obtida da estimativa do gap.

Para desordem do tipo aperiódica, não são esperados efeitos de Griffiths devido a simetria da regra de substituição, pois não há regiões raras e suficientemente grandes para gerar tais efeitos. Assim, tanto o gap quanto o parâmetro de ordem de corda devem se anular no mesmo ponto, eliminando qualquer possibilidade de uma fase de Haldane sem gap como aquela observada em cadeias de spin 1 com desordem aleatória [19]. 


\section{Capítulo 9}

\section{Método de SDRG com correções variacionais.}

Nos capítulos 3 e 4 mostramos que o método de renormalização proposto por Ma, Dasgupta e Hu [11, 12] é baseado em cálculo perturbativos, que não são confiáveis no regime de modulação fraca, ou seja, quando os acoplamentos vizinhos são da mesma ordem do maior acoplamento (acoplamento central). Devido a esse problema e ao interesse em aplicar o SDRG no regime de modulação fraca, em geral para identificar o ponto crítico que separa o regime de modulação fraca do regime de modulação forte, propusemos um tratamento alternativo para obter os parâmetros do hamiltoniano efetivo.

Essa nova proposta surge quando procuramos, para a regra de dizimação, um hamiltoniano efetivo que reproduza da melhor maneira possível os níveis de energia mais baixos do bloco, considerando não apenas o espectro do par de spins central, mas alternativamente o espectro do bloco formado pelo par de spins central e seus vizinhos à esquerda e à direita. O método baseia-se na minimização da diferença entre o espectro do hamiltoniano original e o espectro do hamiltoniano efetivo, onde usamos o hamiltoniano efetivo das regras do SDRG como tentativa inicial. Durante minimização mantemos a forma do hamiltoniano efetivo do SDRG e variamos os parâmetros efetivos para minimizar a diferença entre os espectros de autovalores. 


\section{Modelo de Ising com campo transverso.}

Ilustraremos esse método com o modelo de Ising unidimensional em um campo transverso, pois este é mais simples para se trabalhar analiticamente que o modelo de Heisenberg. Esse modelo em uma cadeia de $\mathrm{N}$ spins é definido pelo hamiltoniano

$$
\mathcal{H}=-\sum_{i=1}^{N-1} J_{i} \sigma_{i}^{x} \sigma_{i+1}^{x}-\sum_{i=1}^{N} b_{i} \sigma_{i}^{z}
$$

onde $\sigma_{i}^{x, z}$ são as matrizes de Pauli de spin $1 / 2$. As variáveis $\left\{J_{i}\right\}$ e $\left\{b_{i}\right\}$ podem ser distribuídas aleatoriamente ou aperiodicamente.

Para introduzir a regra de dizimação do SDRG no caso do modelo estudado aqui, escolhemos os parâmetros $b_{i}$ e $J_{i}$ distribuídos aleatoriamente de acordo com uma determinada distribuição de probabilidades. Consideramos inicialmente que a distribuição seja larga o suficiente para que tenhamos, com alta probabilidade, um acoplamento mais forte $J_{0}$, com acoplamentos vizinhos muito mais fracos, ou seja, $J_{0} \gg J_{r}, J_{l}, b_{r}, b_{l}$.

Neste caso teremos que lidar com blocos do tipo mostrado na figura 9.1. Consideramos ainda que os parâmetros à esquerda e à direita são iguais, $b_{r}=b_{l}=b$ e $J_{r}=J_{l}=J$, pois todos esses valores são muito menores que $J_{0}$. Assim é possível fazer um tratamento analítico do problema. Os spins no par ligado por $J_{0}$, para baixas energias, encontram-se alinhados paralelamente, comportando-se assim como um único spin, no qual é aplicado um campo transverso efetivo. Podemos então substituir esse par de spins por um único spin efetivo $1 / 2$. Os parâmetros efetivos obtidos por um tratamento perturbativo semelhante ao feito no capítulo 3, equação (3.4), são dados por

$$
\begin{array}{r}
b_{M D H}^{\prime}=\frac{b^{2}}{J_{0}}, \\
J_{M D H}^{\prime}=J .
\end{array}
$$

Agora podemos considerar uma modificação no método que, a partir da matriz efetiva do SDRG, dada por

$$
h^{e f}=-b^{\prime} \sigma^{\prime z}-J^{\prime} \sigma_{l}^{\prime x} \sigma^{\prime x}-J^{\prime} \sigma^{\prime x} \sigma_{r}^{\prime x}+U^{\prime},
$$

minimiza o erro associado com a diferença entre os autovalores dessa matriz em relação 


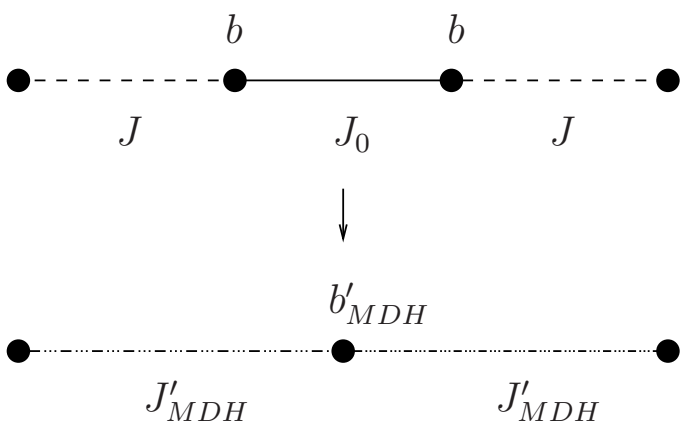

Figura 9.1: Regra de dizimação do SDRG para um bloco com o acoplamento central mais forte no modelo de Ising com campo transverso.

aos menores autovalores da matriz original,

$$
h_{0}=-b\left(\sigma_{1}^{z}+\sigma_{2}^{z}\right)-J \sigma_{l}^{x} \sigma_{1}^{x}-J_{0} \sigma_{1}^{x} \sigma_{2}^{x}-J \sigma_{2}^{x} \sigma_{r}^{x} .
$$

Repare que os spins vizinhos fazem parte dos hamiltonianos, e a partir desse momento são tratados da mesma forma que os spins do par central, não mais como uma perturbação sobre o par central. O parâmetro $U^{\prime}$ é o deslocamento dos níveis de energia que garante que as energias dos estados fundamentais de ambas as matrizes sejam iguais.

O hamiltoniano $h^{\text {ef }}$ tem 4 autovalores duplamente degenerados, designados por $E_{i}^{\prime}$, e o hamiltoniano $h_{0}$ tem 8 autovalores duplamente degenerados, designados por $E_{i}$. Assim definimos uma função erro quadrático médio como

$$
S\left(J^{\prime}, b^{\prime}, U^{\prime}\right)=\sum_{i=0}^{3}\left(E_{i}^{\prime}-E_{i}\right)^{2},
$$

sendo que a soma percorre apenas os primeiros 4 autovalores, pois são esses os autovalores preservados que queremos ajustar.

Devemos encontrar os parâmetros efetivos $J^{\prime}, b^{\prime}$ e $U^{\prime}$ que minimizam essa função. Ou 
seja, precisamos encontrar a solução do sistema de equações

$$
\begin{aligned}
\frac{\partial S}{\partial J^{\prime}} & =2 \sum_{i=0}^{3}\left(E_{i}^{\prime}-E_{i}\right) \frac{\partial E_{i}^{\prime}}{\partial J^{\prime}}=0, \\
\frac{\partial S}{\partial b^{\prime}} & =2 \sum_{i=0}^{3}\left(E_{i}^{\prime}-E_{i}\right) \frac{\partial E_{i}^{\prime}}{\partial b^{\prime}}=0, \\
\frac{\partial S}{\partial U^{\prime}} & =2 \sum_{i=0}^{3}\left(E_{i}^{\prime}-E_{i}\right) \frac{\partial E_{i}^{\prime}}{\partial U^{\prime}}=0 .
\end{aligned}
$$

Os autovalores de $h_{e f}$ são conhecidos analiticamente, apenas para o caso simétrico que estamos estudando, e são dados por

$$
\begin{aligned}
& E_{0}^{\prime}=-\sqrt{b^{2}+4 J^{\prime 2}}+U^{\prime}, \\
& E_{1}^{\prime}=-b^{\prime}+U^{\prime}, \\
& E_{2}^{\prime}=b^{\prime}+U^{\prime}, \\
& E_{3}^{\prime}=\sqrt{b^{\prime 2}+4 J^{\prime 2}}+U^{\prime} .
\end{aligned}
$$

Assim podemos resolver o sistema de equação acima, (9.6), para obter as soluções que minimizam a equação 9.5. Essas soluções são dadas por

$$
\begin{aligned}
b^{\prime} & =\frac{E_{2}-E_{1}}{2}, \\
J^{\prime} & =\frac{1}{2} \sqrt{\left(\frac{E_{3}-E_{0}}{2}\right)^{2}-\left(\frac{E_{2}-E_{1}}{2}\right)^{2}}, \\
U^{\prime} & =\frac{1}{4}\left(E_{0}+E_{1}+E_{2}+E_{3}\right) .
\end{aligned}
$$

No gráfico a seguir, figura 9.2, mostramos a comparação dos parâmetros efetivos calculados pelo SDRG e os calculados pelo procedimento de minimização da diferença entre os autovalores efetivos e originais. Por simplicidade estamos tratando o caso $h=J$ no hamiltoniano original, equação (9.4).

Como esperado, quando os parâmetros $b$ e $J$ têm valores muito menores que $J_{0}$, os parâmetros calculados pelo SDRG e pelo método de minimização coincidem. Estes mesmos parâmetros efetivos diferem muito no limite oposto onde $J, b \sim J_{0}$. 


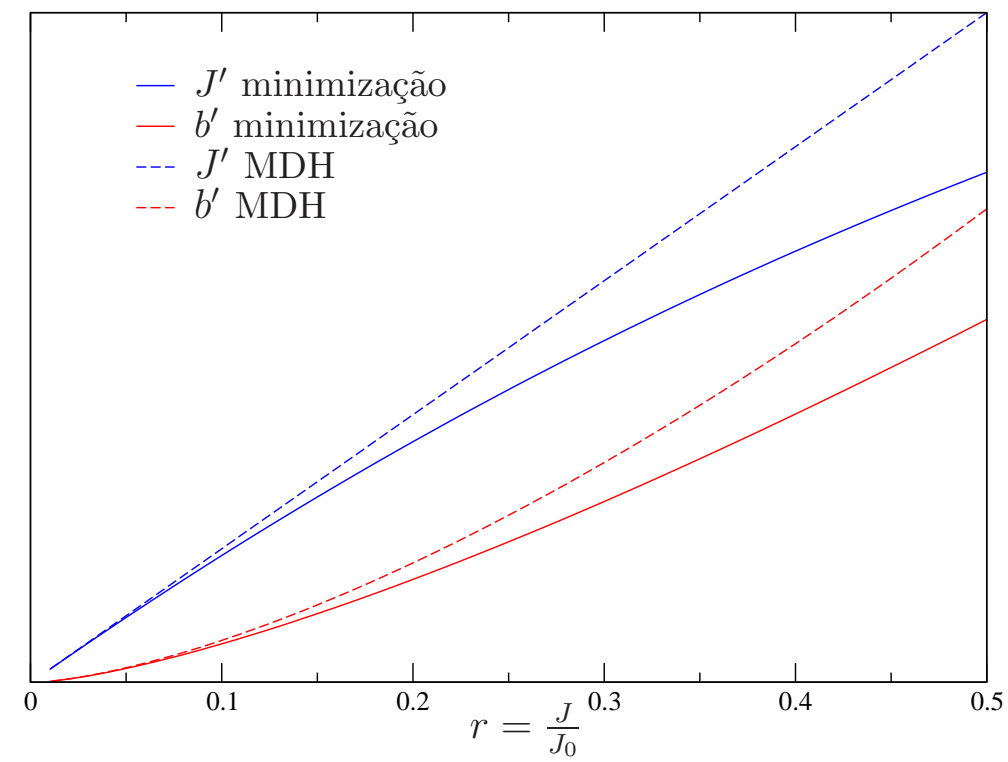

Figura 9.2: Comparação entre os resultados previsto pelo método de SDRG e pela minimização da diferença entre os autovalores originais e efetivos.

Precisamos agora estudar se os novos parâmetros efetivos dão origem a níveis de energia mais fiéis aos níveis de energia da matriz original $h_{0}$, do que os parâmetros efetivos do SDRG. Como pode ser visto na figura 9.3 , para valores onde $J, b \sim J_{0}$, os níveis de energia da matriz com parâmetros efetivos calculados pela minimização são muito próximos daqueles calculados pela diagonalização numérica da matriz original. Os níveis de energia para os parâmetros calculados pelo SDRG são próximos aos níveis do hamiltoniano original apenas no limite $J, b \ll J_{0}$, como esperado.

Quando não dispomos de resultados analíticos para os níveis de energia da matriz efetiva, torna-se impossível calcular os parâmetros efetivos de forma analítica como acabamos de fazer. Porém, ainda podemos minimizar o sistema de equações (9.6) numericamente para encontrar os parâmetros efetivos. Um caso imediato ocorre quando consideramos o problema que acabamos de tratar com parâmetros assimétricos, ou seja, $J_{l} \neq J_{r}$ e $b_{l} \neq b_{r}$.

Com esse método modificado de dizimação local, espera-se que o novo SDRG possa ser aplicado em qualquer região de parâmetros do modelo com desordem. Possibilitando assim a análise de resultados para desordem fraca, pois os parâmetros efetivos não dependem de cálculos perturbativos que não são confiáveis nessa região da desordem. 


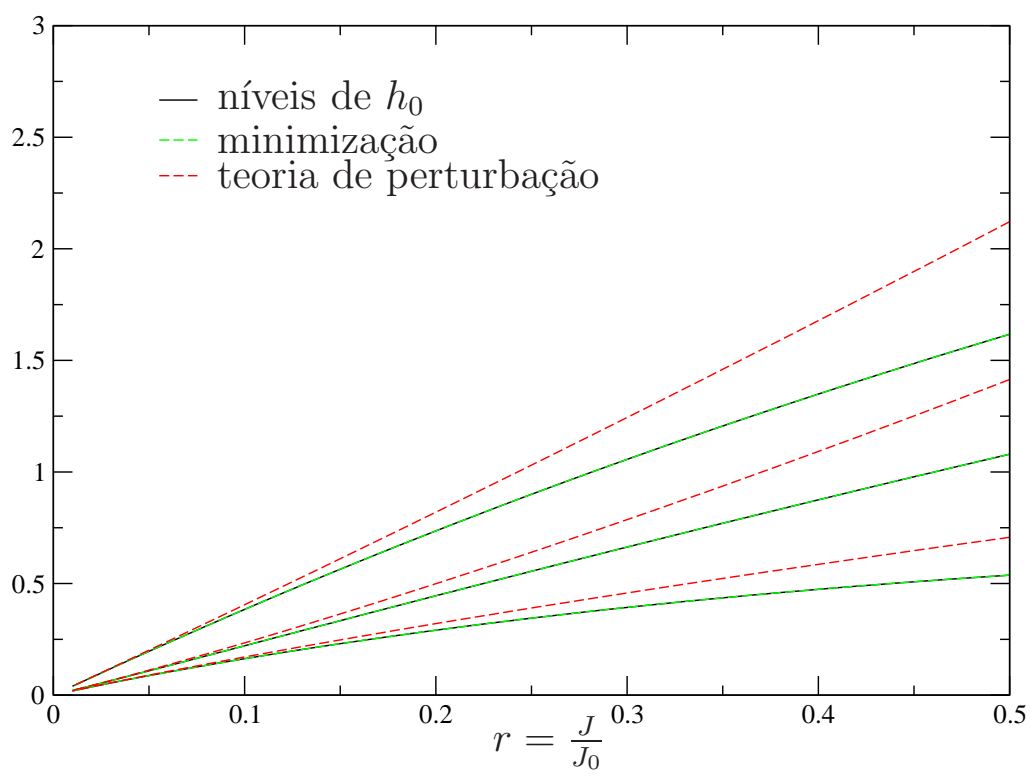

Figura 9.3: Comparação entre os níveis previstos pelo método de MDH e pela minimização.

Porém nossas tentativas em aplicar esse método de renormalização com correções variacionais na cadeia de Ising com campo transverso aperiódica foram improdutivas. Mesmo usando uma formulação um pouco mais geral, para lidar com blocos maiores de spins, esse método não preserva o ponto crítico do modelo, fazendo com que a iteração do RG conduza a cadeia para uma fase ferromagnética, ou para uma fase paramagnética. A sequência aperiódica escolhida foi sequência de Fibonacci $k=2$, definida por $a \rightarrow a b b$, $b \rightarrow a$. Para esse modelo e essa sequência são conhecidos resultados exatos e via SDRG [51]. Isso possibilitaria a análise do quanto o método de SDRG modificado aperfeiçoa os resultados do SDRG tradicional. 


\section{Capítulo 10}

\section{Discussão dos resultados e Conclusões.}

Neste trabalho foram investigados os efeitos causados nas propriedades termodinâmicas de baixas temperaturas da cadeia de Heisenberg de spin 1 pela introdução de desordem aperiódica na distribuição de acoplamentos. Por meio de adaptações do grupo de renormalização de Ma, Dasgupta de Hu (SDRG), apresentamos resultados analíticos para desordem aperiódica gerada por duas sequências aperiódicas, a sequência de Fibonacci e 6-3, sendo que a segunda apresenta flutuações geométricas mais fortes que a primeira.

O desenvolvimento do SDRG empregado aqui envolveu primeiramente a adaptação das regras de renormalização para o caso de desordem aperiódica, onde lidamos em geral com blocos formados por vários spins e não apenas por pares. Desenvolvemos também ferramentas para utilizar não somente a abordagem inicialmente proposta por Monthus et al. [23, 24] (segunda abordagem), mas também para utilizar a abordagem mais geral que leva em conta ligações efetivas longas que cobrem vizinhos distantes (terceira abordagem). Utilizamos ainda a generalização direta do SDRG para cadeias de spin 1 (primeira abordagem). Surpreendentemente, verificamos que mesmo para modulação forte a segunda abordagem nem sempre fornece resultados que concordam com as outras abordagens, enquanto os resultados da primeira e segunda abordagens sempre concordam entre si.

Ainda no limite de modulação forte, a segunda abordagem fornece resultados qualitativamente corretos apenas quando as flutuações geométricas também são fortes, sendo que quantitativamente os resultados ainda são diferentes quando comparados com a primeira 
e terceira abordagens. Quando as flutuações geométricas são fracas, os resultados não concordam nem mesmo qualitativamente.

Especificamente para a cadeia de Fibonacci-Heisenberg, que tem um expoente de flutuação geométrica $\omega=0$, a segunda abordagem mostrou uma transição de fase para uma fase dominada pela aperiodicidade para desordem forte, enquanto a primeira e terceira abordagens previram que o gap de Haldane não seria destruído pela desordem aperiódica mesmo com desordem forte, mostrando assim que, para o caso de flutuações geométricas $\operatorname{com} \omega=0$, as ligações não frustradas entre segundos vizinhos são essenciais na análise do SDRG. Por meio dos cálculos de Monte Carlo quântico foi possível confirmar que quantitativamente o espectro efetivo previsto pela primeira ou terceira abordagens é mais fiel ao espectro da cadeia de Fibonacci-Heisenberg, enquanto os cálculos de DMRG confirmaram que o gap de Haldane sobrevive à desordem de Fibonacci.

Por outro lado, quando a desordem aperiódica segue a sequência 6-3, que tem o expoente de flutuação geométrica dado por $\omega \simeq 0.43$, caracterizando flutuações geométricas muito mais fortes que as flutuações da sequência de Fibonacci, todas as abordagens dão o mesmo resultado qualitativo, onde a fase de Haldane sobrevive para modulação fraca $(r \sim 1)$, enquanto é destruída para modulação forte $(r \gg 1)$. Assim a cadeia de Heisenberg-6-3 sofre uma transição do fase, quando a modulação cresce, para uma fase sem gap dominada pela aperiodicidade, similar àquela observada para cadeias de Heisenberg-6-3 de spin 1/2. Novamente os cálculos de Monte Carlo quântico e de DMRG confirmam os resultados do SDRG, porém, salientamos que os cálculos de QMC novamente mostraram que o espectro efetivo previsto pelas primeira e terceira abordagens é mais fiel ao espectro da cadeia de Heisenberg-6-3 do que o espectro efetivo previsto pela segunda abordagem, figuras 7.9 e 7.10.

Os resultados apresentados aqui para duas sequências aperiódicas, especialmente para as equações de recorrência da razão entre os acoplamentos $r$ (ou $\rho$ ), quando a modulação é forte, estão de acordo com a forma geral proposta no trabalho [34], no contexto das cadeias aperiódicas de spin $1 / 2$, onde para qualquer regra de substituição de duas letras caracterizada por um expoente crítico $\omega \geq 0$, é possível escrever uma equação de recorrência para a razão entre os acoplamentos dada por

$$
r^{(j+1)}=\gamma\left[r^{(j)}\right]^{k}
$$


onde $\gamma$ é uma constante, $r^{(j)}$ é a razão entre os acoplamentos calculada na $j$-ésima iteração do SDRG, $k$ é um inteiro relacionado ao expoente de flutuação geométrica $\omega$ e ao fator de reescala $\tau$ da transformação dado por 1

$$
\omega=\frac{\ln k}{\ln \tau}
$$

Enquanto para a segunda abordagem [23, 24], a constante $\gamma(=2)$ é maior que 1, para as outras abordagens $0<\gamma(=3 / 4)<1$. Para $k \geq 2(\omega>0)$ a razão efetiva diverge iterativamente sempre que a razão inicial $r^{(0)}$ é grande o bastante, independentemente do valor de $\gamma$, o que leva o sistema a uma fase com gap nulo no limite de modulação forte. Por outro lado, quando $k=1(\omega=0)$, a constante $\gamma$ define se a razão efetiva entre os parâmetros diverge, levando o sistema para uma fase sem gap dominada pela aperiodicidade, ou se a razão se aproxima de 1, levando o sistema para a fase de Haldane. Assim, podemos inferir que somente quando o expoente de flutuação geométrica é zero as diferentes abordagens do SDRG conduzirão a resultados qualitativamente diferentes.

Em geral, podemos afirmar que a presença de desordem aperiódica caracterizada por um expoente $\omega$ positivo induz a uma transição de fase, entre a fase de Haldane e uma fase sem gap, quando a razão entre os acoplamentos iniciais $r^{(0)}$ aumenta, enquanto desordem aperiódica com $\omega=0$ não destrói a fase de Haldane. Podemos traçar um paralelo com a cadeia aperiódica de spins $1 / 2$, lembrando que o critério de Harris-Luck prediz alterações na criticalidade para $\omega \geq-\frac{1}{2}$. Nota-se que a desordem de Fibonacci não induz diferenças significativas na cadeia de spin 1, como ocorre no estado fundamental da cadeia de spin $1 / 2$. Também notamos outro contraste quando comparamos nossos resultados com os resultados da cadeia com desordem aleatória de spins 1, pois não há uma fase intermediária associada com singularidades de Griffiths. Isto ocorre pois, nas sequências aperiódicas não há raras regiões, arbitrariamente grandes, nas quais o sistema está localmente em outra fase quando comparado com a cadeia infinita. Isto está de acordo com o comportamento encontrado na cadeia de Ising quântica [51], assim como no contexto do processo de contato aperiódico [52].

Por fim salientamos que, como o ponto crítico encontrado na cadeia de Heisenberg-6-3 corresponde a uma razão inicial de valor baixo, as estimativas via SDRG dos expoentes

\footnotetext{
${ }^{1} \mathrm{O}$ fator de reescala da sequência de Fibonacci é dado por $\tau=2+\sqrt{5}$, equação (5.9). Na sequência 6-3 esse fator é dado por $\tau=5$, equação (6.12).
} 
críticos da transição, que utiliza cálculos perturbativos, são tecnicamente difíceis e não confiáveis, assim como o próprio valor da razão crítica. 


\section{Apêndice A}

\section{Resultados da teoria de perturbação em primeira ordem.}

Os resultados para a teoria de perturbação em primeira ordem, considerando os termos originais da hamiltoniana local para um par de spins 1, equação (4.3), e da hamiltoniana efetiva $h_{1}^{\text {exact }}$, equação (4.8), são dados por

$$
\left\langle m_{l}, \Psi_{0}, m_{r}\left|h_{1}\right| m_{l}^{\prime}, \Psi_{0}, m_{r}^{\prime}\right\rangle=\left\langle m_{l}, \Phi_{0}, m_{r}\left|h_{1}^{\text {exact }}\right| m_{l}^{\prime}, \Phi_{0}, m_{r}^{\prime}\right\rangle=0
$$

para o estado de singleto. Para o estado de tripleto temos como resultado

$$
\begin{aligned}
\left\langle m_{l}, \Psi_{1}^{m}, m_{r}\left|h_{1}\right| m_{l}^{\prime}, \Psi_{1}^{m^{\prime}}, m_{r}^{\prime}\right\rangle & =\left\langle m_{l}, \Phi_{1}^{m}, m_{r}\left|h_{1}^{\text {exact }}\right| m_{l}^{\prime}, \Phi_{1}^{m^{\prime}}, m_{r}^{\prime}\right\rangle= \\
& =\frac{J_{l}}{2 \sqrt{2}}\left\langle m_{l}, m_{r}\left|S_{l}^{+}\right| m_{l}^{\prime}, m_{r}^{\prime}\right\rangle\left(\delta_{m^{\prime}, 0} \delta_{m,-}+\delta_{m^{\prime},+} \delta_{m, 0}\right) \\
& +\frac{J_{l}}{2 \sqrt{2}}\left\langle m_{l}, m_{r}\left|S_{l}^{-}\right| m_{l}^{\prime}, m_{r}^{\prime}\right\rangle\left(\delta_{m^{\prime},-} \delta_{m, 0}+\delta_{m^{\prime}, 0} \delta_{m,+}\right)+ \\
& +\frac{J_{l}}{2}\left\langle m_{l}, m_{r}\left|S_{l}^{z}\right| m_{l}^{\prime}, m_{r}^{\prime}\right\rangle \delta_{m^{\prime}, m}\left(\delta_{m,+}-\delta_{m,-}\right)+ \\
& +\frac{J_{r}}{2 \sqrt{2}}\left\langle m_{l}, m_{r}\left|S_{r}^{+}\right| m_{l}^{\prime}, m_{r}^{\prime}\right\rangle\left(\delta_{m^{\prime}, 0} \delta_{m,-}+\delta_{m^{\prime},+} \delta_{m, 0}\right) \\
& +\frac{J_{r}}{2 \sqrt{2}}\left\langle m_{l}, m_{r}\left|S_{r}^{-}\right| m_{l}^{\prime}, m_{r}^{\prime}\right\rangle\left(\delta_{m^{\prime},-} \delta_{m, 0}+\delta_{m^{\prime}, 0} \delta_{m,+}\right)+ \\
& +\frac{J_{r}}{2}\left\langle m_{l}, m_{r}\left|S_{r}^{z}\right| m_{l}^{\prime}, m_{r}^{\prime}\right\rangle \delta_{m^{\prime}, m}\left(\delta_{m,+}-\delta_{m,-}\right) .
\end{aligned}
$$


Por fim para os elementos de matriz mistos envolvendo estados de singletos e tripletos encontramos

$$
\begin{aligned}
& \left\langle m_{l}, \Psi_{0}, m_{r}\left|h_{1}\right| m_{l}^{\prime}, \Psi_{1}^{m}, m_{r}^{\prime}\right\rangle=\left\langle m_{l}, \Phi_{0}, m_{r}\left|h_{1}^{\text {exact }}\right| m_{l}^{\prime}, \Phi_{1}^{m}, m_{r}^{\prime}\right\rangle=-\frac{J_{l}}{\sqrt{3}}\left\langle m_{l}, m_{r}\left|S_{l}^{+}\right| m_{l}^{\prime}, m_{r}^{\prime}\right\rangle \delta_{m,+} \\
& +\frac{J_{l}}{\sqrt{3}}\left\langle m_{l}, m_{r}\left|S_{l}^{-}\right| m_{l}^{\prime}, m_{r}^{\prime}\right\rangle \delta_{m,-}+\frac{2 J_{l}}{\sqrt{6}}\left\langle m_{l}, m_{r}\left|S_{l}^{z}\right| m_{l}^{\prime}, m_{r}^{\prime}\right\rangle \delta_{m, 0}+\frac{J_{r}}{\sqrt{3}}\left\langle m_{l}, m_{r}\left|S_{r}^{+}\right| m_{l}^{\prime}, m_{r}^{\prime}\right\rangle \delta_{m,+} \\
& \quad-\frac{J_{r}}{\sqrt{3}}\left\langle m_{l}, m_{r}\left|S_{r}^{-}\right| m_{l}^{\prime}, m_{r}^{\prime}\right\rangle \delta_{m,-}-\frac{2 J_{r}}{\sqrt{6}}\left\langle m_{l}, m_{r}\left|S_{r}^{z}\right| m_{l}^{\prime}, m_{r}^{\prime}\right\rangle \delta_{m, 0} .
\end{aligned}
$$

Nas expressões anteriores usamos a seguinte notação

$$
\begin{aligned}
\left|m_{l}, \Psi_{s}^{m}, m_{r}\right\rangle \equiv\left|m_{l}\right\rangle \otimes\left|\Psi_{s}^{m}\right\rangle \otimes\left|m_{r}\right\rangle, \quad \text { com }\left|\Psi_{0}\right\rangle & =\frac{1}{\sqrt{3}}(|+-\rangle-|00\rangle+|-+\rangle), \\
\left|\Psi_{1}^{1}\right\rangle & =\frac{1}{\sqrt{2}}(|+0\rangle-|0+\rangle), \\
\left|\Psi_{1}^{0}\right\rangle & =\frac{1}{\sqrt{2}}(|+-\rangle-|-+\rangle), \\
\left|\Psi_{1}^{-1}\right\rangle & =\frac{1}{\sqrt{2}}(|0-\rangle-|-0\rangle),
\end{aligned}
$$

onde $\left|m_{l}\right\rangle$ e $\left|m_{r}\right\rangle$ são os estados dos primeiros vizinhos do par central, e $\left|\Psi_{s}^{m}\right\rangle$ são estados de $h_{0}$ com spin total $s$ e projeção $m$. Note que estados com $s=2$ não são usados nesse cálculo. Os estados $\left|\Phi_{0}\right\rangle$ e $\left|\Phi_{1}^{m}\right\rangle$ foram definidos na equação (3.3). Portanto $h_{1}^{\text {exact }}$ é de fato a matriz efetiva que resulta da teoria de perturbação de primeira ordem, quando substituímos o par de spins 1 por um par de spins $1 / 2$.

Porém para a hamiltoniana efetiva $h_{1}^{\text {eff }}$, equação (4.7), que não leva em conta as ligações de segundos vizinhos, a igualdade nas equações (A.1) e (A.2) também é verificada, enquanto na equação (A.3) isso não ocorre. No último caso temos o resultado

$$
\begin{array}{r}
\left\langle m_{l}, \Phi_{0}, m_{r}\left|h_{1}^{e f f}\right| m_{l}^{\prime}, \Phi_{1}^{m}, m_{r}^{\prime}\right\rangle=-\frac{J_{l}^{\prime}}{2 \sqrt{2}}\left\langle m_{l}, m_{r}\left|S_{l}^{+}\right| m_{l}^{\prime}, m_{r}^{\prime}\right\rangle \delta_{m,+}+ \\
+\frac{J_{l}^{\prime}}{2 \sqrt{2}}\left\langle m_{l}, m_{r}\left|S_{l}^{-}\right| m_{l}^{\prime}, m_{r}^{\prime}\right\rangle \delta_{m,-}+\frac{J_{l}^{\prime}}{2}\left\langle m_{l}, m_{r}\left|S_{l}^{z}\right| m_{l}^{\prime}, m_{r}^{\prime}\right\rangle \delta_{m, 0}+ \\
+\frac{J_{r}^{\prime}}{2 \sqrt{2}}\left\langle m_{l}, m_{r}\left|S_{r}^{+}\right| m_{l}^{\prime}, m_{r}^{\prime}\right\rangle \delta_{m,+}-\frac{J_{r}^{\prime}}{2 \sqrt{2}}\left\langle m_{l}, m_{r}\left|S_{r}^{-}\right| m_{l}^{\prime}, m_{r}^{\prime}\right\rangle \delta_{m,-}+ \\
-\frac{J_{r}^{\prime}}{2}\left\langle m_{l}, m_{r}\left|S_{r}^{z}\right| m_{l}^{\prime}, m_{r}^{\prime}\right\rangle \delta_{m, 0} .
\end{array}
$$




\section{Apêndice B}

\section{Resultados da teoria de perturbação em segunda e terceira ordem.}

Discutimos aqui detalhes adicionais aos cálculos de teoria de perturbação usados na terceira abordagem, veja a figura 5.3(b). O cálculo do parâmetro efetivo que surge da dizimação do par de spins $(2,3)$ envolve cálculos de teoria de perturbação de segunda ordem, como aqueles mostrados na seção 3, equação (3.4), ou seja, a regra 2. Porém temos ligações adicionais de segundos vizinhos, que geram mais elementos no termo perturbativo, veja a equação (4.8). Na teoria de perturbação de segunda ordem há um termo quadrático do elemento perturbativo. Esse termo quadrático dá origem a quatro contribuições que podem ser interpretadas como na figura B.1. onde se ilustram as quatro possíveis combinações, duas a duas, das ligações perturbativas.

(a)
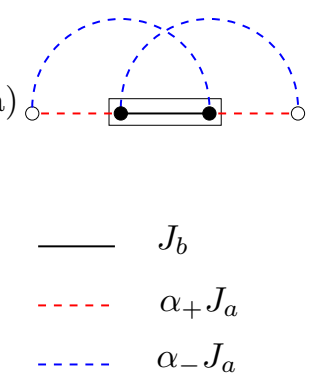

(b)

(c)

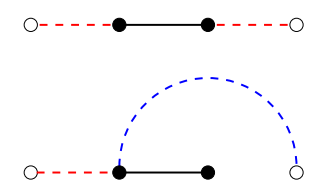

(d)

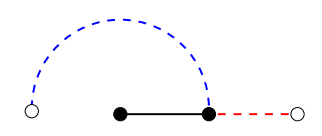

(e)

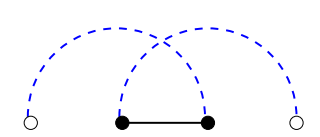

Figura B.1: Ilustração das diferentes contribuições que surgem na utilização da regra 2, quando há ligações de segundos vizinhos conectando o par central e o restante da cadeia. 
Ao aplicar a cada uma dessas combinações a regra 2 encontramos o acoplamento efetivo

$$
J_{b}^{\prime}=\left(\alpha_{+}-\alpha_{-}\right)^{2}\left(\frac{1}{2}\right) \frac{J_{a}^{2}}{J_{b}}=\left(\frac{4}{3}\right) \frac{J_{a}^{2}}{J_{b}} .
$$

Para o segundo parâmetro efetivo, aquele entre os spins 4 e 9 , precisamos evitar a dizimação sequencial dos pares conectados por $J_{b}$, pois a presença de ligações que conectam somente os dois pares fortemente acoplados causa mudanças no valor de $J_{b}$, aumentando a escala de energia, o que fere a auto-consistência do método de SDRG. Evitamos esse problema dizimando ao mesmo tempo os dois pares de spins $1 / 2$ conectados por $J_{b}$, usando teoria de perturbação em terceira ordem, pois a contribuição de segunda ordem também se anula nesse caso. Assim consideramos as correções no estado fundamental dos dois pares, equação (5.17), dado por $\left|\Psi_{0}\right\rangle=\left|\Phi_{0}\right\rangle_{56} \otimes\left|\Phi_{0}\right\rangle_{78}$ devido ao seguinte termo perturbativo

$$
\begin{aligned}
h_{1}^{\text {exact }} & =J_{a} \mathbf{S}_{4} \cdot\left(\alpha_{+} \mathbf{s}_{5}+\alpha_{-} \mathbf{s}_{6}\right)+J_{a} \mathbf{s}_{5} \cdot\left(\alpha_{+} \alpha_{-} \mathbf{s}_{7}+\alpha_{-}^{2} \mathbf{s}_{8}\right)+ \\
& +J_{a} \mathbf{s}_{6} \cdot\left(\alpha_{+}^{2} \mathbf{s}_{7}+\alpha_{+} \alpha_{-} \mathbf{s}_{8}\right)+J_{a}\left(\alpha_{-} \mathbf{s}_{7}+\alpha_{+} \mathbf{s}_{8}\right) \cdot \mathbf{S}_{9},
\end{aligned}
$$

que inclui as ligações de segundos e terceiros vizinhos da hamiltoniana local.

De acordo com a teoria de perturbação, a correção de terceira ordem é por

$$
\begin{aligned}
h^{\text {eff }} & =\sum_{i \neq 0, j \neq 0} \frac{\left\langle\Psi_{0}\left|h_{1}^{\text {exact }}\right| \Psi_{i}\right\rangle\left\langle\Psi_{i}\left|h_{1}^{\text {exact }}\right| \Psi_{j}\right\rangle\left\langle\Psi_{j}\left|h_{1}^{\text {exact }}\right| \Psi_{0}\right\rangle}{\left(E_{i}-E_{0}\right)\left(E_{j}-E_{0}\right)}+ \\
& -\left\langle\Psi_{0}\left|h_{1}^{\text {exact }}\right| \Psi_{0}\right\rangle \sum_{i \neq 0} \frac{\left|\left\langle\Psi_{0}\left|h_{1}^{\text {exact }}\right| \Psi_{i}\right\rangle\right|^{2}}{\left(E_{i}-E_{0}\right)^{2}},
\end{aligned}
$$

na qual os estados são obtidos de produtos diretos dos autoestados dos pares de spin 5-6 e 7-8. Estes estados são: o estado fundamental $\left|\Psi_{0}\right\rangle=\left|\Phi_{0}\right\rangle_{56} \otimes\left|\Phi_{0}\right\rangle_{78}$, formado pela combinação dos estados de singletos definidos na equação (3.2), e os estados excitados $\left|\Psi_{i}\right\rangle$ que são formados pelas combinações de singleto-tripleto ou tripleto-tripleto dos estados $\left|\Phi_{0}\right\rangle,\left|\Phi_{1}^{+}\right\rangle,\left|\Phi_{1}^{0}\right\rangle$, e $\left|\Phi_{1}^{-}\right\rangle$; veja novamente (3.3).

Esta somatória resulta no acoplamento efetivo entre os spins 4 e 9 dado por

$$
J_{a}^{\prime}=\left(\alpha_{+}-\alpha_{-}\right)^{4}\left(\frac{1}{2}\right)^{2} \frac{J_{a}^{3}}{J_{b}^{2}}=\left(\frac{4}{3}\right)^{2} \frac{J_{a}^{3}}{J_{b}^{2}} .
$$




\section{Apêndice $\mathrm{C}$}

\section{SDRG: Cálculo do gap para cadeias finitas de Fibonacci-Heisenberg.}

Uma estimativa do SDRG para o gap em uma cadeia finita pode ser obtida interrompendose o esquema do SDRG no menor nível de energia para o qual ainda há ao menos dois spins ativos. As figura de C.1 até C.3 ilustram este procedimento para cadeias com $L=14, L=22$ e $L=35$ spins. Como queremos obter estimativas para comparar com os resultados de DMRG da seção 8.1, precisamos considerar o gap entre o estado fundamental, com spin total $S_{T}=0$ ou $S_{T}=1$, e o nível mais baixo com $S_{T}=2$. Para $L=14$ e $L=22$, o estado fundamental é um singleto $\left(S_{T}=0\right)$, enquanto para $L=35$, com um número ímpar de spins, o estado fundamental tem spin total $S_{T}=1$.

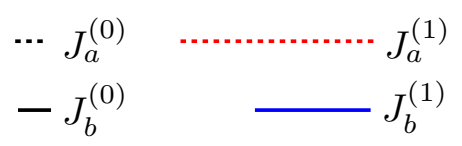

(a)

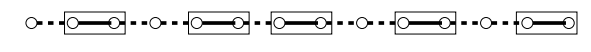

(b)

Figura C.1: Abordagem do SDRG aplicado na cadeia de Fibonacci-Heisenberg de spin 1 com $L=14$ sítios. Após uma varredura em (b), todos os spins são eliminados.

Como ilustra a figura C.1, para $L=14$ spins, o estado de mais baixa energia $S_{T}=2$ corresponde a dois pares de spins excitados no estado $S=1$. Assim, a estimativa para este gap é $2 J_{b}^{(1)}$, com o acoplamento efetivo $J_{b}^{(1)}$ calculado da equação Eq. (5.3). Para 


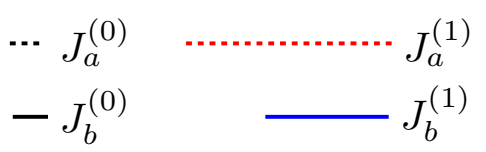

(a)

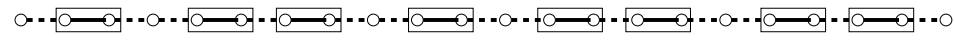

(b)

(c)

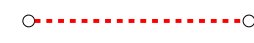

Figura C.2: Abordagem do SDRG aplicado na cadeia de Fibonacci-Heisenberg de spin 1 com $L=22$ sítios. Após uma varredura em (b), todos as ligações $J_{b}$ são eliminadas, deixando apenas uma única ligação $J_{a}$ como mostra a figura (c).

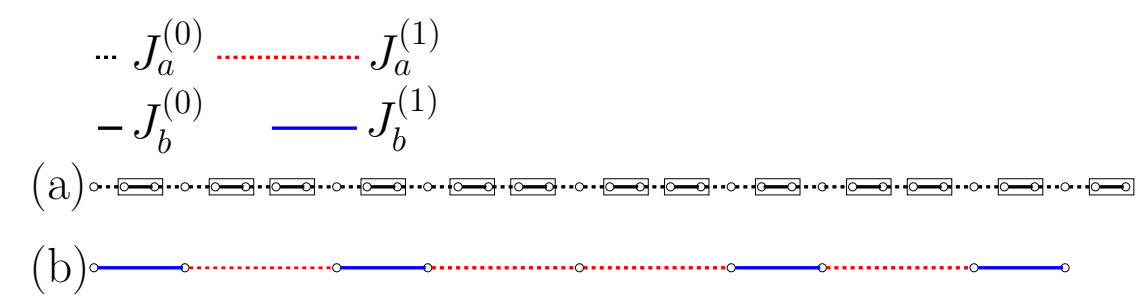

Figura C.3: Abordagem do SDRG aplicado na cadeia de Fibonacci-Heisenberg de spin 1 com $L=35$ sítios. Após uma varredura em (b), apenas o spins central sobrevive.

$L=22$, como ilustra a figura C.2, o estado excitado de energia mais baixo $S_{T}=2$ vem de um estado excitado $S=2$ de um par de spins conectados por um acoplamento $J_{a}^{(1)}$, dando origem à um gap $3 J_{a}^{(1)}$. Finalmente, como o estado fundamental para $L=35$ tem spin total $S_{T}=1$, a excitação de mais baixo energia $S_{T}=2$ corresponde a uma excitação local de um único par de spins, levando a um gap de $J_{b}^{(1)}$, como mostra a figura C.3. Note que isto explica o comportamento não monotônico dos gaps com o aumento do tamanho do sistema, para $r \gtrsim 5$, como observado para 8.1 .

Estimativas para o gap via SDRG para valores maiores de $L$ podem ser obtidos de maneira análoga. 


\section{Referências Bibliográficas}

[1] F. Alet, S. Wessel, and M. Troyer, Phys. Rev. E 71, 036706 (Mar 2005)

[2] A. Albuquerque, F. Alet, P. Corboz, P. Dayal, A. Feiguin, S. Fuchs, L. Gamper, E. Gull, S. Gürtler, A. Honecker, R. Igarashi, M. Körner, A. Kozhevnikov, A. Läuchli, S. Manmana, M. Matsumoto, I. McCulloch, F. Michel, R. Noack, G. P. owski, L. Pollet, T. Pruschke, U. Schollwöck, S. Todo, S. Trebst, M. Troyer, P. Werner, and S. Wessel, J. Magn. Magn. Mater. 310, 1187 (2007), ISSN 0304-8853

[3] C. Payen, E. Janod, K. Schoumacker, C. D. Batista, K. Hallberg, and A. A. Aligia, Phys. Rev. B 62, 2998 (Aug 2000)

[4] I. Affleck, J. Phys.: Condens. Matter 1, 3047 (1989)

[5] G. Xu, J. F. DiTusa, T. Ito, K. Oka, H. Takagi, C. Broholm, and G. Aeppli, Phys. Rev. B 54, R6827 (Sep 1996)

[6] F. D. M. Haldane, Phys. Rev. Lett. 50, 1153 (1983)

[7] I. Affleck, T. Kennedy, E. H. Lieb, and H. Tasaki, Phys. Rev. Lett. 59, 799 (1987)

[8] M. den Nijs and K. Rommelse, Phys. Rev. B 40, 4709 (Sep 1989)

[9] K. Hida, J. Phys. Soc. Jpn. 68, 3177 (1999)

[10] S. R. White, Phys. Rev. Lett. 69, 2863 (1992)

[11] S.-K. Ma, C. Dasgupta, and C.-K. Hu, Phys. Rev. Lett. 43, 1434 (1979)

[12] C. Dasgupta and S.-K. Ma, Phys. Rev. B 22, 1305 (1980) 
[13] R. N. Bhatt and P. A. Lee, Phys. Rev. Lett. 48, 344 (Feb 1982)

[14] C. A. Doty and D. S. Fisher, Phys. Rev. B 45, 2167 (1992)

[15] D. S. Fisher, Phys. Rev. B 50, 3799 (1994)

[16] P. Henelius and S. M. Girvin, Phys. Rev. B 57, 11457 (1998)

[17] N. Laflorencie, H. Rieger, A. W. Sandvik, and P. Henelius, Phys. Rev. B 70, 054430 (2004)

[18] J. A. Hoyos, A. P. Vieira, N. Laflorencie, and E. Miranda, Phys. Rev. B 76, 174425 (2007)

[19] K. Damle, Phys. Rev. B 66, 104425 (2002)

[20] A. Saguia, B. Boechat, and M. A. Continentino, Phys. Rev. Lett. 89, 117202 (2002)

[21] R. A. Hyman and K. Yang, Phys. Rev. Lett. 78, 1783 (1997)

[22] R. A. Hyman, Ph.D. thesis, Indiana University (1996)

[23] C. Monthus, O. Golinelli, and T. Jolicoeur, Phys. Rev. Lett. 79, 3254 (1997)

[24] C. Monthus, O. Golinelli, and T. Jolicoeur, Phys. Rev. B 58, 805 (1998)

[25] S. Bergkvist, P. Henelius, and A. Rosengren, Phys. Rev. B 66, 134407 (2002)

[26] P. Lajkó, E. Carlon, H. Rieger, and F. Iglói, Phys. Rev. B 72, 094205 (2005)

[27] Y. Nishiyama, Physica A 252, 35 (1998)

[28] K. Hida, Phys. Rev. Lett. 83, 3297 (1999)

[29] K. Yang and R. A. Hyman, Phys. Rev. Lett. 84, 2044 (2000)

[30] S. Todo, K. Kato, and H. Takayama, J. Phys. Soc. Japan 69A, 355 (2000)

[31] R. B. Griffiths, Phys. Rev. Lett. 23, 17 (1969)

[32] D. Schechtman, I. Blech, D. Gratias, and J. W. Cahn, Phys. Rev. Lett. 53, 1951 (1984) 
[33] A. P. Vieira, Phys. Rev. Lett. 94, 077201 (2005)

[34] A. P. Vieira, Phys. Rev. B 71, 134408 (2005)

[35] H. L. Casa Grande, N. Laflorencie, F. Alet, and A. P. Vieira, Phys. Rev. B 89, 134408 (2014)

[36] C. Janot, Quasicrystals - A Primer (Clarendon Press Oxford, 1992)

[37] S. T. R. Pinho and T. C. P. Lobão, Braz. J. Phys. 30, 772 (2000)

[38] M. Queffélec, Substitutional dynamics systems - Spectral Analysis, volume 1294 of Lec- tures Notes in Mathematics (Springer-Verlag, 1987)

[39] A. B. Harris, J. Phys. C 7, 1671 (1974)

[40] J. M. Luck, Europhys. Lett. 24, 359 (1993)

[41] M. Kossow, P. Schupp, and S. Kettemann, Int. J. Mod. Phys. Conf. Ser. 11, 183 (2012)

[42] J. Hermisson, J. Phys. A: Math. Gen. 33, 57 (2000)

[43] F. Iglói and C. Monthus, Phys. Rep. 412, 277 (2005)

[44] K. Hida, Phys. Rev. B 45, 2207 (1992)

[45] A. Sandvik, AIP Conf. Proc. 1297, 135 (2010)

[46] A. Sandvik and J. Kurkijärvi, Phys. Rev. B 43, 5950 (1991)

[47] O. F. Syljuåsen and A. Sandvik, Phys. Rev. E 66, 046701 (2002)

[48] A. Sandvik, J. Phys A. 25, 3667 (1992)

[49] I. P. McCulloch, J. Stat. Mech, P10014(2007)

[50] I. P. McCulloch and M. Gulácsi, Europhys. Lett. 57, 852 (2002)

[51] F. J. Oliveira Filho, M. S. Faria, and A. P. Vieira, J. Stat. Mech. 2012, P03007 (2012)

[52] H. Barghathi, D. Nozadze, and T. Vojta, Phys. Rev. E 89, 012112 (2014) 